Florida International University

FIU Digital Commons

$10-28-2019$

\title{
The Influence of Maternally Regulated Prenatal Sensory Experience on Postnatal Motor Coordination in Neonatal Bobwhite Quail
}

Starlie C. Belnap

Florida International University, sbeln001@fiu.edu

Follow this and additional works at: https://digitalcommons.fiu.edu/etd

Part of the Biological Psychology Commons, Cognition and Perception Commons, Developmental Psychology Commons, and the Development Studies Commons

\section{Recommended Citation}

Belnap, Starlie C., "The Influence of Maternally Regulated Prenatal Sensory Experience on Postnatal Motor Coordination in Neonatal Bobwhite Quail" (2019). FIU Electronic Theses and Dissertations. 4358.

https://digitalcommons.fiu.edu/etd/4358

This work is brought to you for free and open access by the University Graduate School at FIU Digital Commons. It has been accepted for inclusion in FIU Electronic Theses and Dissertations by an authorized administrator of FIU Digital Commons. For more information, please contact dcc@fiu.edu. 


\section{FLORIDA INTERNATIONAL UNIVERSITY}

Miami, Florida

\section{THE INFLUENCE OF MATERNALLY REGULATED PRENATAL SENSORY EXPERIENCE ON POSTNATAL MOTOR COORDINATION IN NEONATAL BOBWHITE QUAIL (COLINUS VIRGINIANUS)}

A dissertation submitted in partial fulfillment of the requirements for the degree of DOCTOR OF PHILOSOPHY

in

PSYCHOLOGY

by

Starlie C. Belnap 
To: Dean Michael R. Heithaus

College of Arts, Sciences and Education

This dissertation, written by Starlie C. Belnap, and entitled The Influence of Maternally Regulated Prenatal Sensory Experience on Postnatal Motor Coordination in Neonatal Bobwhite Quail (Colinus Virginianus), having been approved in respect to style and intellectual content, is referred to you for judgment.

We have read this dissertation and recommend that it be approved.

$\begin{array}{r}\text { Eliza Nelson } \\ \hline \text { Bethany Reeb-Sutherland } \\ \hline \text { Edgar R. Vieira } \\ \hline \text { Robert Lickliter, Major Professor }\end{array}$

Date of Defense: October 28, 2019

The dissertation of Starlie C. Belnap is approved.

Dean Michael R. Heithaus

College of Arts, Sciences and Education

Andrés G. Gil

Vice President for Research and Economic Development and Dean of the University Graduate School

Florida International University, 2019 
(C) Copyright 2019 by Starlie C. Belnap

All rights reserved. 


\section{DEDICATION}

This is dedicated to David, my patient husband, and my wonderful children

Brayden, Ashton and Corbin. Without your support, sacrifice, encouragement and most of all love I would not have made it this far. 


\section{ACKNOWLEDGMENTS}

Special thanks to:

Dr. Robert Lickliter, my mentor, advisor, and extremely talented quail whisper

Committee members:

Dr. Eliza Nelson

Dr. Bethany Reeb-Sutherland

Dr. Edgar R. Vieira

All the Developmental Psychobiology lab members:

Without whom this project would have taken a lifetime to complete. Thank you for your invaluable support, smiles, and unfailing commitment to excellence. You are truly my forever "quail family."

Also, thanks to:

Dr. Stefany Coxe for your love of statistics and your valuable data analysis expertise.

Michele Ramos, for the countless hours you listened and gave encouragement.

Christopher Clifford, for all the yummy treats and your unfailing friendship.

Emily Boeving, for being there when I felt like giving up.

This project was primarily funded through a National Science Foundation grant BCS

1525371 awarded to RL and a Department of Psychology Seed Fund. 


\begin{abstract}
OF THE DISSERTATION
THE INFLUENCE OF MATERNALLY REGULATED PRENATAL SENSORY EXPERIENCE ON POSTNATAL MOTOR COORDINATION IN NEONATAL
\end{abstract} BOBWHITE QUAIL (Colinus virginianus) by

Starlie C. Belnap

Florida International University, 2019

Miami, Florida

Professor Robert Lickliter, Major Professor

Comparative animal studies aid in understanding how prenatal sensory experiences regulated by maternal activity facilitate or interfere with growth and phenotype development. However, there is a paucity of information on how prenatal sensory experience influence postnatal motor performance. In the current studies, we used an avian model, the bobwhite quail, to evaluate the effects of prenatal temperature (study 1), prenatal movement (study 2), prenatal light duration (study 3), and prenatal light presentation pattern (study 4) on hatchability, growth and postnatal motor performance in $24 \mathrm{hr}$ quail neonates. In study 1 , quail embryos were exposed to naturally occurring cool $\left(36.9^{\circ} \mathrm{C}\right)$ or warm $\left(38.1^{\circ} \mathrm{C}\right)$ temperatures for a brief 4-day period during early incubation. In study 2, quail embryos were exposed to increased or decreased egg turning experience during the second week of incubation. In study 3 , quail embryos were exposed to 6hrs or $2 \mathrm{hrs}$ of crepuscular light stimulation during the final days of incubation. In study 4 , quail embryos were exposed to either sporadic or crepuscular light patterns for either $2 \mathrm{hrs}$ or 6hrs durations during the final days of incubation. All studies, except for Study 4, were 
compared to control chicks who had received industry standard care throughout incubation, which included optimal temperature exposure of $37.5^{\circ} \mathrm{C}$, movement every 2 hours, and light exposure for a $12 \mathrm{hr}$ on/off cycle. Motor performance, body mass and tarsometatarsus length (indicators of growth) were evaluated $24 \mathrm{hrs}$ after hatching. Cool thermal exposure delayed hatching, reduced body mass, and decreased motor performance. Warm thermal exposure delayed bone growth and increased fall frequency. Decreased movement exposure delayed hatching, reduced body mass, and delayed motor performance. Increased movement exposure delayed motor performance and reduced body mass, but did not delay hatching. Crepuscular light exposure negatively influenced growth and decreased motor performance, but only delayed hatching in the $2 \mathrm{hr}$ crepuscular treatment. Lastly, results from study 4 demonstrated sporadic light presentation improved gait performance in $2 \mathrm{hr}$ exposed chicks and improved growth indicators in $6 \mathrm{hr}$ exposed chicks. The present studies provide evidence that maternally regulated prenatal experience contributes to postnatal motor performance and highlights the importance of non-obvious stimulation for typical motor development. Keywords: avian embryos, motor development, prenatal temperature, prenatal light, prenatal movement, egg turning, neurodevelopment, kinematics, motor coordination, locomotion 


\section{TABLE OF CONTENTS}

CHAPTER

PAGE

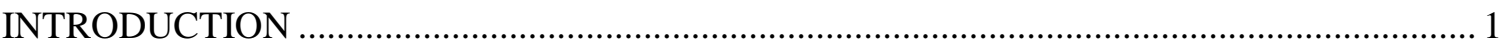

Study 1: PRENATAL INCUBATION TEMPERATURE AFFECTS NEONATAL PRECOCIAL BIRDS' LOCOMOTOR BEHAVIOR

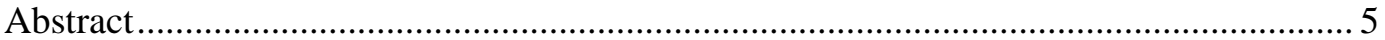

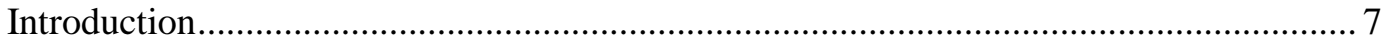

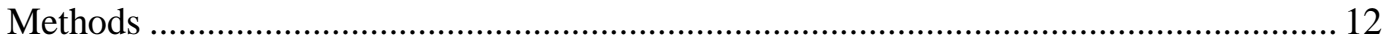

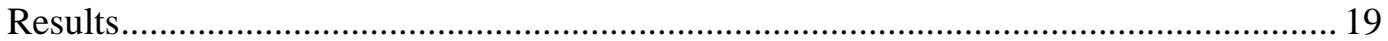

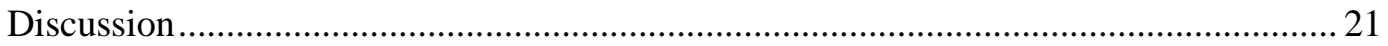

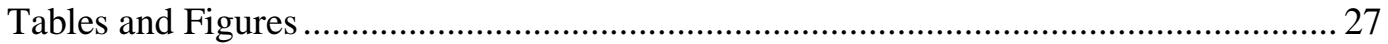

Study 2: EFFECTS OF INCREASED AND DECREASED EARLY INCUBATION MOVEMENT ON NEONATAL PRECOCIAL BIRDS' LOCOMOTOR BEHAVIOR .............. 33

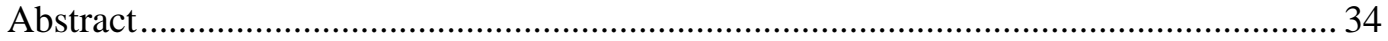

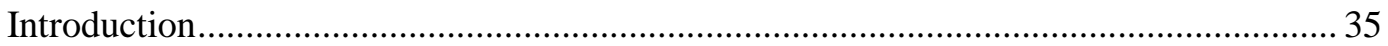

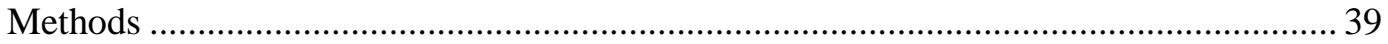

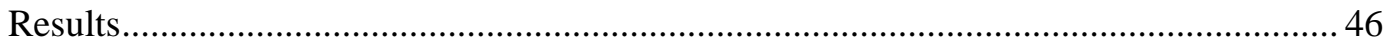

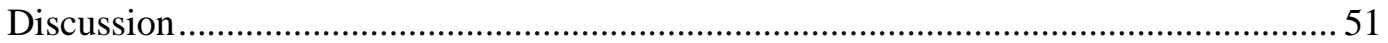

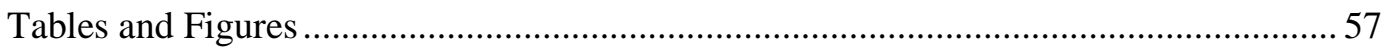

Study 3 \& 4: PRENATAL LIGHT EXPOSURE INFLUENCES GAIT PERFORMANCE AND

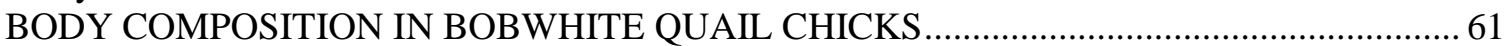

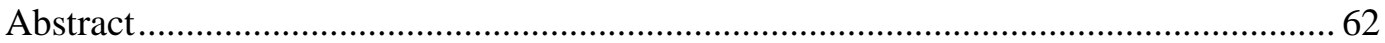

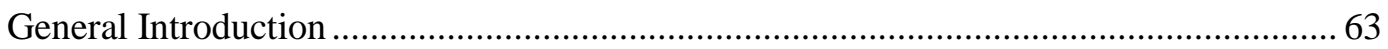

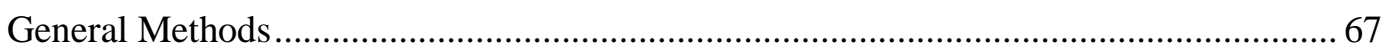

Study 3: The effect of discontinuous prenatal light exposure on body composition and

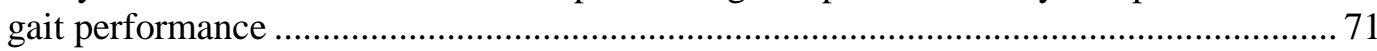

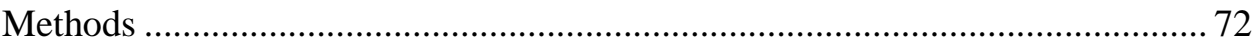

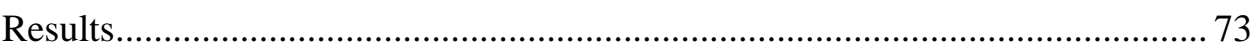

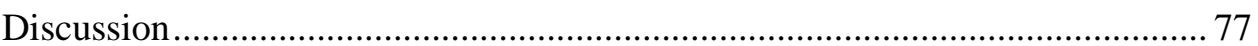

Study 4: The effect of prenatal light exposure duration and presentation pattern on body

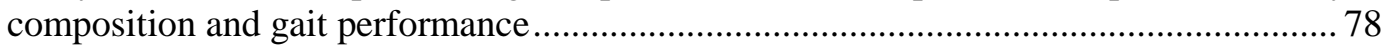

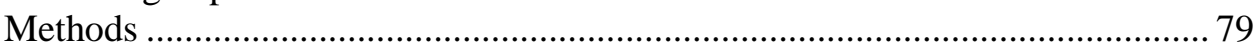

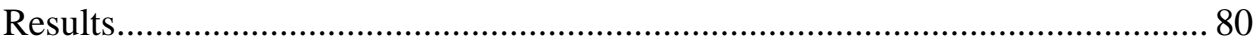

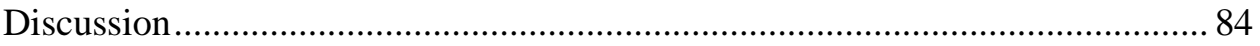

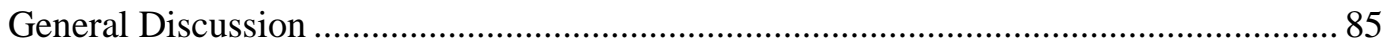

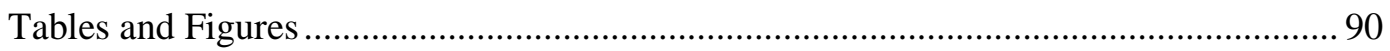

CONCLUSION

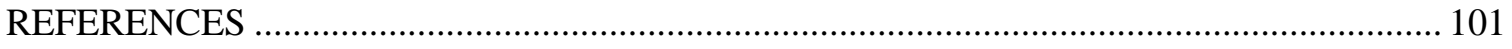

VITA 


\section{INTRODUCTION}

Movement is an important feature of typical development. The very act of moving affords opportunities for intrinsic learning and exploration (Adolph \& Berger, 2006; Piaget, 1951). In Piaget's well known theory of intelligence, infant's overt physical actions scaffold the foundation of representational thought. Similarly, Kelso viewed selforganizing activity as the motor driving self-identity, cognition, and social development (Kelso, 2016). Within the Gibsonian perceptive, acting and perceiving share a reciprocal relationship, where the dimensions and properties of the environment constrain the actions of the organism, and the organism's actions produce not only changes in the environment but also changes in the organism itself (Adolph \& Kretch, 2015; Gibson, 1979; Greeno, 1994). Bushnell and Boudreau argued that the extent a specified motor ability is limited would likewise correspond to a restriction in perceptual ability which may have cascading effects on future developmental trajectories (Bushnell \& Boudreau, 1993). In each of these perspectives, the activity of the organism guides and constrains the developmental process, situating motor ability as a central dynamic component of perceptual, social, and cognitive development.

Extensive research confirms a tight coupling between motor ability and perceptual, social, and cognitive outcomes (Blair \& Raver, 2012; Clearfield et al., 2008; Diamond, 2000; Leonard, 2016; Leonard \& Hill, 2014; Piek et al., 2008). For example, in typically developing children, cognitive processing speed and memory are influenced by gross motor ability in early childhood (Piek et al., 2008), infant social bids for caregivers' attention increase with the onset of crawling and walking (Clearfield et al., 2008), and object perception and recognition are enhanced with the onset of infant 
reaching, grasping, and sitting (Sommerville et al., 2005; Soska et al., 2010). Needham and colleagues (Sommerville et al., 2005) designed a clever 'sticky mitten' paradigm which facilitated object exploration in typically developing pre-grasping three-month-old infants using Velcro mittens and toys. By removing a typical motor constraint, infants improved in object goal behavior and showed improved understanding of others' actions (Sommerville et al., 2005). More recent work has confirmed repeated opportunities for infant reaching facilitates goal directed visual perception and increased skill acquisition (Williams et al., 2015).

Similarly, motor deficits, characteristic of several neurological disorders, commonly correspond to social, perceptual and cognitive deficits. For example, children with Developmental Coordination Disorder (DCD) have deficits in self-regulatory skills, body self-awareness, and social engagement (Lloyd et al. , 2006). Children with Autism Spectrum Disorder (ASD) have difficulty with sensory perception, social engagement, and cognitive flexibility (Lloyd et al., 2013). While both of these disorders are typically diagnosed during early childhood between the ages of 3 to 5 years, subtle differences are present during infancy (Johnson et al., 2016; Nickel et al., 2013; Zwicker et al., 2012). Specifically, research has identified subtle deficits in posture and ocular control in highrisk ASD infants which corresponded to delays in visual-spatial perception (Johnson et al., 2016; Nickel et al., 2013) suggesting subtle motor differences during infancy may set the course for long-term developmental deficits seen in ASD. Interestingly, ASD children who received motor skill training emphasizing dynamic balance, upper and lower limb coordination, and strength conditioning, showed greater body control (Catama et al., 2017) and marked improvement in cognition (Woo \& Leon, 2013). In another 
example, ASD young adults benefited from dance therapy focusing on developing mirror motor neurons. During the intervention, participants took turns mirroring body movements of the instructor, other participates, and leading a group dance. After only 7 sessions, participants showed positive improvements in body awareness, self-other awareness, empathy, and general social skills (Koch et al., 2015).

Altogether, early motor and rehabilitation research provides evidence for a compelling argument that motor development facilitates a range of skills. Despite the importance of motor development, few studies have investigated prenatal factors which may facilitate optimal postnatal motor development. Since muscle contractions are evident within the first few weeks of human gestation (within the first few days for small animals) (de Vries et al., 1982; Hamburger \& Balaban, 1963), and are susceptible and responsive to sensory stimulation (Robinson, 2005; Sindhurakar \& Bradley, 2012; Wu et al., 2001), it is likely these early movements lay the neurological foundation for motor responsiveness and subsequent sensory system functioning (Gottlieb, 1971; Lickliter, 2011; Turkewitz \& Kenny, 1982). However, little is known about what experiences are necessary during the prenatal period to promote healthy motor development. The use of animal models offers an essential step in addressing which prenatal factors are important for optimal postnatal motor development.

In the current series of studies, we used an animal model, the bobwhite quail, to investigate how key factors provided by the nesting hen influenced hatchability, growth, and motor performance. Bobwhite quail are a precocial birds that can walk within hours after hatching and require minimal maternal post hatch care. However, like most avian embryos, quail embryos require extensive prenatal care. In bobwhite quail, egg care is 
provided by the hen. In addition to nest site selection, nest building, and protection from predation, the maternal hen's behavior regulates egg incubation temperature, movement experience, and access to light for the growing embryos. Because of the accessibility of the quail egg, these factors can be experimentally manipulated independent of the mother with strict precision and control. By leveraging these unique developmental conditions, we evaluated how small fluctuations in temperature influenced hatchability, growth, and motor performance (study1), how decreases or increases in egg turning influenced hatchability, growth, and motor performance (study 2), and how duration and presentation of light (study $3 \& 4$ ) influenced hatchability, growth, and motor performance. 


\title{
Study 1
}

\section{PRENATAL INCUBATION TEMPERATURE AFFECTS NEONATAL PRECOCIAL BIRDS' LOCOMOTOR BEHAVIOR}

This manuscript was published by the Journal of Physiology and Behavior July 2019

\author{
Starlie C. Belnap, M.S.*, John P. Currea, M.S., Robert Lickliter, Ph.D. \\ Department of Psychology, Florida International University, Miami, FL 33199, USA
}

*Corresponding author: Starlie Belnap, sbelnap@ fiu.edu, 305-348-1230, Department of Psychology, Florida International University, Miami, FL 33199.

\section{Acknowledgements}

This research was supported by NSF grant BCS 1525371 awarded to RL. We thank Eliza Nelson, Bethany Reeb-Sutherland and Edgar Vieira for constructive comments on the manuscript. We also thank all the student researchers who assisted with this project.

\begin{abstract}
Temperature during the prenatal period is an important factor for developing embryos. Extensive human and animal research indicate embryos are sensitive to small fluctuations
\end{abstract}


in temperature which has profound effects on phenotype development. Much of this research has focused on survivability, morphology, and incubation duration, but comparatively less in known about how prenatal temperature influences the development of motor coordination. In this study, we experimentally tested whether exposure to naturally occurring $\operatorname{cool}\left(36.9^{\circ} \mathrm{C}\right)$ or warm $\left(38.1^{\circ} \mathrm{C}\right)$ thermal conditions for a brief period (4 days) during early incubation can influence postnatal motor performance in neonatal bobwhite quail hatchlings. We compared gait spatiotemporal parameters, body kinematics, and locomotive behaviors of control chicks incubated in an optimal thermal environment $\left(37.5^{\circ} \mathrm{C}\right)$ with thermally manipulated chicks. Experimental temperature treatment began on embryonic day five (E5) and ended on E8. Chicks were tested 24hours after hatching. Cool thermal exposure during incubation delayed hatching, reduced body mass, and increased fall frequency, intertarsal joint angle and stride length variability during the gait task compared to optimally incubated chicks. Warm thermal exposure during incubation delayed bone growth and increased fall frequency relative to controls. We discuss the relationship between motor development and thermal regulatory processes and provide insight into how spatiotemporal parameters aid in elucidating subtle differences in coordinated movement which may contribute to atypical motor development and be associated with neural developmental disorders. We provide the first spatiotemporal evidence for the importance of optimal thermal microclimates for typical prenatal motor development.

Keywords: avian embryos, motor development, prenatal temperature, neurodevelopment, kinematics, motor coordination 


\section{Introduction}

It is well known that temperature during the prenatal period is an important factor for developing embryos. Temperature has pervasive effects on sex, morphology, physiology and behavior (Durant et al., 2010; DuRant et al., 2008; Rhen \& Lang, 2004). Different parental behaviors and regulatory processes have emerged to help ensure offspring survivability and fitness under various thermal conditions (Cockburn, 2006; Collias \& Eollias, 1984; Cooke et al., 2003). In humans, the uterine environment acts as a heat dispersal system helping to lower fetal basal temperature (Baumgart, 2008). In reptiles and birds, the ovo nest environment acts as a thermal insulator and parental behavior regulates heat and protection from predation (Collias \& Eollias, 1984; Kolbe \& Janzen, 2002; Orcutt \& Orcutt, 1976). However, disruptions in thermal regulatory processes and behaviors can result in structural and functional defects depending on when and how long the insult occurs during embryo development (DuRant et al., 2010; Edwards, 2006; Webb, 1987).

For reptiles, nest site selection and environmental conditions largely determine prenatal incubation temperature, resulting in considerable fluctuations during development. These fluctuations during critical periods determine gonadal sex, with low temperatures $\left(\sim 26^{\circ} \mathrm{C}\right)$ producing high ratios of females and high temperatures $\left(\sim 32^{\circ} \mathrm{C}\right)$ producing high ratios of males (Mrosovsky \& Pieau, 1991; Rhen \& Lang, 2004). In addition to temperature-dependent sex, small changes in incubation temperature $\left(<2^{\circ} \mathrm{C}\right)$ in reptiles affect hatchling morphology, growth, and locomotor performance (Booth, 2006; Rhen \& Lang, 2004). Embryos incubated under cooler conditions take longer to hatch, and embryos incubated under warmer conditions typically have larger body mass 
and longer appendages (e.g. snout, tail, and limb) (Elphick \& Shine, 1998; Mrosovsky \& Pieau, 1991; While et al., 2018) Broadly, warmer prenatal temperatures improve locomotor performance for turtles and lizards (Booth, 2006; Elphick \& Shine, 1998; While et al., 2018), but in most studies developmental age, time spent in the age prior to hatch, was not considered, even though differences were reported.

In contrast to most reptiles, avian parents actively regulate incubation by investing considerable time and energy into nest building and maintenance (Cockburn, 2006; Collias \& Eollias, 1984; Jr \& Orcutt, 1976; Martin et al., 2007). Parental effort substantially reduces temperature fluctuations experienced by developing embryos (Carroll et al., 2018; DuRant et al., 2013), making thermal-regulatory parental behaviors a key factor in phenotype development and behavioral plasticity. Similar to reptiles, small differences in incubation temperature can profoundly influence avian incubation periods (Hepp et al., 2006; Martin et al., 2007), with warmer temperatures resulting in shorter incubation periods and cooler temperatures producing longer incubation periods (Deeming \& Ferguson, 1992; French, 1997; Hepp et al., 2006). Temperature also affects locomotor performance, with cooler temperatures resulting in a decline in performance (Hopkins et al., 2011) but an increase in quick incomplete explorative behaviors (Hope et al., 2018). Again, developmental age was not considered in these studies. The influence of small temperature differences on growth parameters (e.g. body mass, limb length) is less clear. Duck embryos incubated under consistently optimal thermal conditions $\left(37^{\circ} \mathrm{C}\right)$ produced larger body mass compared to medium $\left(35.9^{\circ} \mathrm{C}\right)$, and low $\left(35^{\circ} \mathrm{C}\right)$ conditions, but did not show differences in limb length (DuRant et al., 2010). In contrast, Hopkins and colleagues (2011) failed to detect differences in body mass, but did detect differences 
in limb length. Embryos in the medium temperature condition had longer limb lengths compared to optimal and cooler temperature conditions (Hopkins et al., 2011), whereas chicken embryos who experienced only 4 days of increased prenatal incubation temperature $\left(38.5^{\circ} \mathrm{C}\right)$ during early development showed both increased body mass and limb length (Hammond, et al., 2007).

Similar to avian models, mammal embryos are protected from thermal fluctuations by the mother. Her thermoregulatory system works as a defense from invading pathogens (Kluger et al., 1996) and helps to regulate important bodily circadian rhythms (Brown et al., 2002). Naturally occurring oscillating body temperature is closely associated with locomotor activity. Small variations in behavioral patterns are important because prenatal activity mediates musculoskeletal formation and lays the neurological foundation for motor responsiveness and subsequent sensory system function (Gottlieb, 1971; Hammond et al., 2007; Hepper, 2015; Lickliter, 2011; Turkewitz \& Kenny, 1982). The majority of mammalian research has focused on the teratogenic effects of extreme temperature $\left(>2^{\circ} \mathrm{C}\right)$ on embryo development. As a result, comparatively less is known about how small prenatal temperature fluctuations $\left(<2^{\circ} \mathrm{C}\right)$ influence morphological, neurobehavioral, and motor development in infants. However, infant rat studies investigating the influence of mild increased postnatal temperature on limb growth provide some evidence of the likelihood that small prenatal thermal changes may influences prenatal growth in mammals (Racine et al., 2018; Serrat, 2014). Moreover, the effects of extreme prenatal temperature on developing embryos provides evidence that prenatal temperature does influence key systems needed for optimal motor development. These teratogenic effects have been extensively documented in endotherms, with notable 
damage occurring to the central nervous and musculoskeletal systems (Edwards, 1986; Edwards et al., 1995; Lieberman et al., 2000; Zerbo et al., 2013).

Although research has demonstrated the influence of typical and atypical prenatal temperature on survivability, morphology, and incubation duration, comparatively less is known about how prenatal temperature affects the development of motor coordination. To date, most of the literature has focused on reptilian locomotor performance as measured by maximum speed on a "racetrack" (Booth, 2006; Du \& Ji, 2003; Elphick \& Shine, 1998; Van Damme et al., 1992). However, motor performance in ectothermic animals is particularly sensitive to environmental thermal conditions at the time of test (Guderley, 2004). Further, lizard embryos incubated at higher temperatures hatched nearly ten days sooner than those incubated under moderate thermal conditions (Elphick \& Shine, 1998; Van Damme et al.,1992). Longer incubation length results in more yolk being converted into tissue (Booth, 2018; Ischer et al., 2009), potentially changing the composition and relative frequency of the muscle fibers (Booth, 2018). Therefore, differences in developmental age might explain the observed differences in reported locomotor performance (Elphick \& Shine, 1998; Van Damme et al., 1992). Hopkins and colleagues (2011) provide evidence that prenatal temperature influences locomotor performance in ducks, the only known study in endothermic animals. However, like reptilian locomotor research, this study used maximum speed to measure locomotor performance and did not account for developmental age.

Speed alone is not an adequate measure of motor performance, especially in bipedal locomotion. Gait analysis provides comprehensive information about spatiotemporal components of locomotion and can be used in conjunction with body 
kinematics to evaluate the integration of muscle and joint function to maintain posture during forward motion (Murray, 1967). Specific spatial measurements such as stride length (distance traveled between foot falls of the same limb) and step width (distance between the left and right foot during forward locomotion) provide insight into the base of support, while joint angles provide information about the body's range of motion (see Figure 1 \& 2) (Andrada et al., 2013; Biewener \& Daley, 2007; Gatesy \& Biewener, 1991; Hausdorff, 2007). Variability in these gait parameters provides information about neuromotor control and fall risk (Hausdorff, 2005, 2007; Stroobants et al., 2013). Measurements such as cadence (steps per second) and fall frequency provide information about the speed and flexibility of the nervous system to compensate for stability perturbations (Hausdorff, 2007; You et al., 2001). This detailed approach to evaluating motor performance may provide insight into specific subtle motoric differences potentially modulating postnatal outcomes and contribute to atypical behavior often seen in human developmental disorders.

The present study provides the first experimental examination of prenatal temperature on the development of postnatal motor coordination in bobwhite quail hatchlings. The primary aim of this study was to determine if prenatal temperature influences postnatal motor coordination. Avian research has suggested that developing avian embryos are sensitive to fluctuations in temperature and respond to warmer thermal conditions with increased prenatal activity (Hammond et al., 2007), shorter incubation periods (Hepp et al., 2006; Martin et al., 2007), and better locomotor performance (Hopkins et al., 2011), whereas colder thermal conditions produce lengthened incubation periods (Hepp et al., 2006; Suarez et al., 1996). Given these findings, we predicted chicks 
incubated under a brief period of naturally occurring warm incubation temperature would show enhanced motor coordination, while chicks incubated in a brief period of cold temperature would show decreased motor coordination relative to controls. Additionally, because some studies showed either an increase in body mass or limb length when incubated under warmer thermal conditions, we anticipated chicks in the warmer thermal condition would show increased body composition (i.e. body mass, tarsometatarsus length) relative to controls and colder thermal conditions.

\section{Methods}

\section{Animal and incubation}

Two hundred and twenty-five fertilized bobwhite quail eggs were obtained from a commercial game bird supplier (Strickland) over the course of nine weeks. Each week 25 eggs were randomly selected and placed into one of three digital temperature and humidity-controlled automatic turning portable incubators (King R-com). Each incubator provided one of three prenatal temperature conditions: low $\left(36.9^{\circ} \mathrm{C} \pm 0.2^{\circ} \mathrm{C}\right)$, control $\left(37.5^{\circ} \mathrm{C} \pm 0.2^{\circ} \mathrm{C}\right)$, and high $\left(38.1{ }^{\circ} \mathrm{C} \pm 0.2^{\circ} \mathrm{C}\right)$ during the manipulation period. Before and after the manipulation period, all incubators were maintained at $37.5^{\circ} \mathrm{C} \pm 0.2^{\circ} \mathrm{C}$, the optimal temperature for hatchability (Wilson et al., 1979). The temperature manipulation period was selected following the same design as described by Hammond and colleagues (2007), with slight adjustments for species differences in incubation length. The incubation period for chickens typically is 21 days while bobwhite quail incubation period is 23 days. To account for these differences and to accurately reflect the same developmental period as described by Hamburger and Hamilton (Ainsworth et al., 2010; Hamburger \& Hamilton, 1951), where extensive myoblast proliferation and limb 
differentiation occurs, the temperature manipulation period began on embryonic day five (E5) and continued through E8 (4 days). The temperatures selected corresponded with the upper and lower tolerance range reported for the common quail (Webb, 1987; Wilson et al., 1979).

The day fertile eggs were placed in an incubator was recorded as day zero and eggs remained in their assigned incubator until E21 when they were transferred to a hatcher maintained at $37.5^{\circ} \mathrm{C} \pm 0.2^{\circ} \mathrm{C}$ until day of hatching (E23). The incubators and hatcher were maintained at $65 \% \pm 5 \%$ humidity throughout the incubation period. To ensure the stability of temperature and humidity, the incubators and hatcher were monitored every four hours throughout the day.

All incubators and the hatcher were housed within a specially designed customizable stimulation cabinet $(0.61 \mathrm{~m} \times 0.61 \mathrm{~m} \times 1.72 \mathrm{~m})$ which allowed for control of auditory and visual experience. The cabinet consisted of four equal white chambers $(0.61 \mathrm{~m} \times 0.61 \mathrm{~m} \times 0.43 \mathrm{~m})$ with independent maintenance doors. Each cabinet was outfitted with LED strip lighting $(4.5 \mathrm{~m})$ which was independently controlled by Samsung Smartthings controllers located outside the cabinet. Light for each cabinet was maintained on the same 12-hour light/dark cycle throughout the incubation period.

On day 15 , the eggs were candled to identify infertile eggs and non-developed embryos. Because we wanted to control for developmental age, chicks who hatched one day early (E22) or one day late (E24) were considered off-cycle hatchlings and were not tested. Only chicks who hatched on day 23 were tested the following day, at approximately 24 hours of age.. Chicks' distal phalanges were marked for individual identification (traditional leg bands were avoided to prevent confounds during motor 
testing) and were transferred and separated by treatment to clear plastic rearing tubs in clutches of 12-15 chicks to simulate naturally occurring brood conditions. Rearing tubs were maintained within a sound-attenuated rearing room at $30^{\circ} \mathrm{C}$ on a 12 -hour light/dark cycle with food and water available ad libitum. All hatchling testing and care followed approved procedures and protocols reviewed by the Institutional Animal Care and Use Committee.

\section{Gait Task and Body Composition Measures}

Hatchling motor coordination was evaluated using a 5-minute gait task one day following hatch. The 5-minute gait task consisted of a 1-minute acclimation period within a dark emergence box $(10 \mathrm{~cm} \times 10 \mathrm{~cm} \times 10 \mathrm{~cm})$ followed by a $4-$ minute free roam exploration period within a Plexiglass runway. One hour prior to testing, square reflective tape $(.5 \times .5 \mathrm{~cm})$ was placed in the center of the right and left tarsal pads to enhance visibility of foot placement during video scoring. To decrease handling effects and stress, chicks were transported in a plastic opaque container to the testing runway. Only chicks who emerged from the box were evaluated for motor coordination (see Table 1).

Following testing, chicks were immediately returned to the rearing room, where they were weighed and the right tarsometatarsus (TARS) (Figure 1) length was measured. TARS length was measured as the distance between the notch at the intertarsal joint angle (ITJ) and the juncture of the TARS and the first digit.

The entire 5-minute testing session occurred within a Plexiglas rectangle runway outfitted with a dark removable lid $(95 \mathrm{~cm}$ long x $13 \mathrm{~cm}$ wide x $20 \mathrm{~cm}$ tall) raised approximately one meter above the floor. The Plexiglas rectangle runway sat within a wooden support frame housed in a temperature-controlled room $\left(30^{\circ} \mathrm{C} \pm 1^{\circ} \mathrm{C}\right)$. A black 
foamboard fake floor suspended approximately $40 \mathrm{~cm}$ below the runway was fitted around a black USB camera (60fps 1230x 720dpi) which was connected to a computer located in an adjacent room. The floor hid the inferior camera from the hatchlings view and provided the visual effect of a solid floor. A second white USB camera was affixed on the white wall outside the runway to provide a lateral view and was connected to the same computer. The inside of the runway was divided into three sections: start area (15 $\mathrm{cm}$ long), runway ( $65 \mathrm{~cm}$ long), and speaker area $(15 \mathrm{~cm} \mathrm{long})$. The start area contained the emergence box which remotely opened after the 1-minute acclimation period. The speaker area located opposite from the start area contained a speaker calibrated at $65 \mathrm{~dB}$ which continuously played a bobwhite quail maternal call to motivate hatchlings to cross the runway during the test period. The lateral and inferior camera views were recorded using Kinovea, an open source kinematic software program.

\section{Gait parameters}

Inferior videos were behaviorally scored using Datavyu (Datavyu Team, 2014), a quantitative event-recording behavioral program, for frequency of forward steps and falls. Steps were defined as controlled forward movement, (walk, ground run, or aerial run) relative to orientation of the hatchling's head (Belnap \& Lickliter, 2017). This definition excluded lateral movement (i.e. side steps used in turning), movement with no distance traveled (i.e. shifting of center of gravity from foot to foot), and backwards movement (relative to chick orientation not the speaker area). Hatchlings were scored as falling, a measurement of instability, when their upper thorax or head contacted with the runway's floor. Hatchlings in a crouched position, knees and hip joints flexed with the thorax 
resting on the ground, were not scored as a fall. Step cadence was defined as the ratio of steps per seconds of active movement.

Spatiotemporal parameters and body kinematics including stride length, step width, stance width, velocity, and ITJ (see Figure $1 \&$ Figure 2) were measured using Kinovea. Inferior videos were evaluated for each parameter on separate frame by frame viewing passes until 20 usable measurements of each parameter were collected. The supporting wooden frame was used as a reference for measurement calibration. Stride length, step width, and stance width were scored using the inferior view. Velocity and ITJ angles were scored using the lateral camera view. Chicks who failed to emerge from the start box or who failed to produce more than 10 useable data points because of lack of movement were excluded from analysis (see Table 1).

For stride length and step width, only successfully completed gait cycles were used to calculated averages. Measurements for stride length were acquired at touch down for each limb throughout the entire video. Stance width, distance between left and right limb during standing, was acquired during moments of rest. Rest was defined as $180(\sim 3$ seconds) frames of non-movement, including forward movement, falls, jumps, and other forms of instability.

Velocity and ITJ angles were evaluated using the lateral view and calibrated using the wood support frame. ITJ was measured at initial touchdown of the gait cycle to capture maximum leg extension. Measurements occurred when the hatchling's locomotion was perpendicular to the camera, providing a clear profile view without video distortion of the right limb. Approximately 20 useable frames were acquired throughout the video for each bird with a minimum of 10 useable frames needed to be included in the 
analysis. ITJ angles were estimated by drawing two digital reference lines within Kinovea. The first line began at the juncture of the TARS and the third digit and followed the length of the TARS. The second line began at the notch of the ITJ and extended up the lateral side of the body approximating the position of the tibiotarsus (see Figure 1). The base of the digital protractor tool within Kinovea was placed on the first reference line and then extended to the second reference line producing the estimated protraction ITJ angle per stride for the right limb. Velocity was calculated only during bouts of

forward locomotion using the following formula, $v=\frac{x_{2}-x_{1}}{t_{2}-t_{1}}$, where $\mathrm{x}$ represents the bird's position and t represents time for each frame within a bout. Locomoting bouts were scored manually by starting and stopping the tracking feature within Kinovea. A bout initiated at toe off and ended at touchdown of a sequence of forward locomotion. In the event a bird became unstable during the sequence, the locomoting bout concluded the gait cycle prior to the instability. Velocity reflected the change in position over the change in time averaged across all locomotion bouts.

Four observers were assigned to each performance measure. Prior to scoring, observers were trained until inter- and intra-rater reliability of $>85 \%$ was achieved. Upon completion of scoring, roughly $10 \%$ of videos were randomly selected to be rescored to verify accuracy for each measure. Observers were blind to temperature condition and relied upon individual bird identification markings, which were void of treatment assignment, to report results.

\section{Data Analysis}

Spatiotemporal data per subject per parameter were averaged and the coefficient of variation ( $\mathrm{CV}=\mathrm{SD} / \mathrm{mean} * 100)$ was calculated (Searls, 1964), providing the 
percentage of variability for ITJ, stride length, step width, and stance width, resulting in a total of eight measures (i.e. stride length, CV stride length, step width, CV step width, stance width, CV stance width, ITJ, and CV ITJ). All behavioral (step cadence and fall frequency) and spatiotemporal parameters were bootstrapped with replacement for 2,000 iterations. Right and left limb measurements for stride length were evaluated using paired sample t-tests. Chi-square likelihood tests and simple binomial outcome tests were used to characterize the influence of temperature on hatchability and gait task emergence. Differences in body composition and velocity between temperature conditions were evaluated using one-way analysis of variance (ANOVA) and were controlled for in subsequent analysis. Spatiotemporal parameters and behavior were analyzed using multivariate analyses of variance (MANOVA; three temperature conditions on eight gait measures and two behavior measures) with body composition and velocity listed as covariates. MANOVA analysis accounts for potential correlations between dependent variables allowing for differences to be assessed without increasing type I error (Warne, 2014). Significant gait variables were further analyzed using univariate Bonferroni post hoc analyses with alpha adjustment set at p-values 0.015 . Since the frequency of falls may be attributed to differences in activity between temperature treatments, the relationship between step cadence and fall frequency was evaluated using Pearson correlation. If the preceding relationship was significant then step cadence was included as an additional covariate in post hoc analyses for falls. All statistical tests were conducted using SPSS v.22. Statistical significance was set at alpha $.05 .95 \%$ confidence intervals and Cohen's D (Cohen, 1988) effect sizes were calculated and interpreted using guidelines suggested by Durlak (2009). 


\section{Results}

\section{Descriptive Results}

A total of 38 eggs were removed during the candling process with no significant differences between conditions (see Table 1). A total of 154 chicks hatched and 22 offcycle chicks were excluded from the study. In the low treatment group, eight chicks hatched early and seven chicks hatched late. Eight chicks hatched early in the control condition and one chick hatched early in the high treatment condition. Chi-square comparisons showed significant difference in off-cycle hatches by temperature treatment $\left(X^{2} \mathrm{df}(2)=12.25 \mathrm{p}<0.01\right)$, with $28 \%$ of off-cycle hatches occurring in the low treatment condition, $15 \%$ in the control treatment condition, and $2 \%$ occurring in the high treatment condition. Chi-square results comparing probability of early, late, and on-time hatches by temperature treatment revealed significant proportional differences $\left(X^{2} \mathrm{df}(4)=20.235\right.$ $\mathrm{p}<0.001)$. When evaluating late hatches, no significant differences were detected $\left(\mathrm{X}^{2}\right.$ $\mathrm{df}(2)=5.76 \mathrm{p}=0.056)$. However, early hatches were significantly more likely to occur in the cooler temperature condition when compared to chance using an exact binomial test (p<.001 95\%CI: [.76 - 1]). No significant differences between temperature treatments were detected for on-time hatches $\left(\mathrm{X}^{2} \mathrm{df}(2)=.985 \mathrm{p}=0.611\right)$. All conditions were equally likely to exit the emergence box $\left(X^{2} \mathrm{df}(2)=2.312 \mathrm{p}=0.315\right)$ and explore the runway $\left(\mathrm{X}^{2}\right.$ $\operatorname{df}(2)=2.36 \mathrm{p}=0.307)$.

Evaluations of TARS length $(\mathrm{F}(2,88)=4.066, \mathrm{p}<0.05)$ and body mass $(\mathrm{F}(2,88)=3.5, \mathrm{p}<0.05)$ revealed significant differences between groups. Hatchlings incubated in warmer temperatures showed moderately smaller TARS length compared to $\operatorname{control}\left(\Delta \mathrm{M}=-0.089, \mathrm{SE}=0.037, \mathrm{p}<0.015, \mathrm{~d}_{\text {cohen's }}=0.615,[95 \% \mathrm{CI}:-0.16,-0.016]\right)$ and 
cooler temperature $\left(\Delta \mathrm{M}=-0.091, \mathrm{SE}=0.037, \mathrm{p}<0.015, \mathrm{~d}_{\text {cohen's }}=0.610,[95 \% \mathrm{CI}:-0.16,-\right.$ 0.013]) hatchlings (Figure 3). Cool incubated hatchlings weighed significantly less compared to high temperature hatchlings $(\Delta \mathrm{M}=-0.358, \mathrm{SE}=0.146, \mathrm{p}<0.015$,

$d_{\text {cohen's }}=0.785,[95 \%$ CI: $\left.-0.679,-0.039]\right)$, and trended towards weighing less compared to control hatchlings $\left(\Delta \mathrm{M}=-0.325, \mathrm{SE}=0.152, \mathrm{p}=0.036, \mathrm{~d}_{\text {cohen's }}=0.517,[95 \% \mathrm{CI}:-0.647,-\right.$ $0.002])$.

\section{Gait Parameters}

A one-way ANOVA with gait velocity as the dependent measure and temperature condition as the independent variable revealed velocity did not significantly differ between temperature conditions (see Table 2). A paired t-test comparison between right and left limb stride length for each temperature treatment revealed no significant differences between limbs. Subsequent analysis included only the right limb values since TARS length and ITJ angles were only acquired for the right limb. Pearson correlation analysis revealed a significantly large negative relationship between step cadence and number of falls $(\mathrm{r}=-0.606, \mathrm{p}<0.001,95 \% \mathrm{CI}[-0.72--.47])$. Across all temperature treatments, hatchlings decreased in falls as step cadence increased (see Figure 4). MANOVA with temperature as the independent variable and the gait parameters as the dependent measures revealed temperature modestly contributed to the observed differences after controlling for body composition and velocity (Pillai’s Trace $=0.423$ $\left.\mathrm{F}(2,20)=1.88, \mathrm{p}<0.05, \mathrm{\eta}_{\mathrm{p}}{ }^{2}=0.211\right)$. Temperature significantly contributed to observed differences in mean ITJ angle $\left(\mathrm{F}(2,78)=4.247, \mathrm{p}<.05, \mathrm{\eta}_{\mathrm{p}}{ }^{2}=0.10\right)$, stride length $\mathrm{CR}(\mathrm{F}(2$, $\left.78)=4.38, \mathrm{p}<.05, \mathrm{\eta}_{\mathrm{p}}{ }^{2}=0.10\right)$, and number of falls $\left(\mathrm{F}(2,78)=5.594, \mathrm{p}<.05, \mathrm{\eta}_{\mathrm{p}}{ }^{2}=0.13\right)$ as 
illustrated in Figure 5 and Table 2. No significant differences were discovered for other gait parameters (see Table 2).

Univariate post hoc evaluations with body composition and velocity as covariates (step cadence was added when evaluating fall frequency) demonstrated chicks in the low temperature treatment had significantly larger ITJ angles $(\Delta \mathrm{M}=16.671, \mathrm{SE}=5.861$, $\mathrm{p}<0.015, \mathrm{~d}_{\text {cohen's }}=0.634,[95 \%$ CI: 2.330, 31.012]), substantially more variability in stride length $\left(\Delta \mathrm{M}=5.432, \mathrm{SE}=1.85, \mathrm{p}<0.05, \mathrm{~d}_{\text {cohen's }}=0.54\right.$, [95\% CI: 0.906, 9.958]), and greater falls frequency $\left(\Delta \mathrm{M}=9.09, \mathrm{SE}=3.474, \mathrm{p}<0.05, \mathrm{~d}_{\text {cohen's }}=0.93,[95 \% \mathrm{CI}: 0.59,17.59]\right)$ when compared to control treatment chicks (Figure 5 and Table 2). Chicks in the warm temperature treatment demonstrated only an increase in falls $(\Delta \mathrm{M}=9.89, \mathrm{SE}=3.19$, $\left.\mathrm{p}<0.01, \mathrm{~d}_{\text {cohen's }}=0.73,[95 \% \mathrm{CI}: 2.094,17.68]\right)$. Chicks in the warm and cool temperature conditions displayed similar fall rates, contrary to our original predictions.

\section{Discussion}

In this study, we provide the first evidence of the effect of incubation temperature on the development of motor coordination in 24-hour old bobwhite quail hatchlings. Our results indicated that quail embryos incubated under altered temperatures showed a notable perturbation in postnatal motor instability beyond differences in body composition, velocity, and step cadence. Chicks in the low temperature condition showed extensive stride length variability, increased falls, and larger ITJ angle compared to controls, but performed similarly as controls for step cadence and other gait measurements. Like reports from previous research, low temperature treated hatchlings showed a delay in hatching and a reduction in total body mass, but TARS length was unaffected. Contrary to our prediction, embryos who experienced high prenatal 
temperatures showed an increase in falls and shorter TARS length following hatching compared to controls. While not significant, they also showed slightly larger ITJ angle and stride length variability (see Table 2). Thus, our study identifies incubation temperature as a parental effect that can contribute to poor motor coordination after only brief exposure to naturally occurring temperature fluctuations.

Generally, for very young crouching birds with a pronograde trunk like the bobwhite quail, stiffed legged running maximizes range of motion, assists with stability, and produces the most efficient stride (Andrada et al., 2013). A major component to achieve stiff legged running is joint angle of the hip, knee, and tarsal joint (Andrada et al., n.d.). Protraction angles, angles produced from the hip, knee, and tarsal joints at touchdown, do not depend on body size or speed, rather they stay relatively constant (Abourachid, 2001). Increased stride length observed with acceleration typical occurs by increasing the retraction angles. However, protraction angles are tightly related to step length such that the mean protraction angle predicts the average step length (Abourachid, 2001). Therefore, for small birds to achieve speed and stability, they need to have longer effective legs at touchdown and shorter effective legs at lift-off, all while maintaining angular momentum about the center of mass. Specifically, the ITJ acts as the spring in forward locomotion and functions like a damper (Abourachid, 2001; Andrada et al., 2013) dissipating energy to avoid over acceleration of the center of mass, thereby stabilizing the trunk (Andrada et al., 2014). In the current study, temperature did not influence velocity, step cadence, or average stride length, but differences were present in ITJ angles. The significantly large ITJ angles observed in the cool treatment and the slightly larger ITJ angles in the warm treatment may correspond with a stiffer leg unit, 
making it more difficult to dissipate forward momentum. This failure to dissipate acceleration would lead to destabilization and potentially result in more falls.

Another alternative to the observed differences in ITJ angle may be related to the trunk posture of the birds. In crouching birds with a pronograde trunk, the center of mass is located forward of the base of support, making joint angle adjustments the primary method for trunk and head stabilization during terrestrial locomotion (Abourachid et al., 2011; Andrada et al., 2014; Nyakatura et al., 2014). If cool and warm temperature birds showed a cranial shift in the center of mass, then an increase in the effective leg length would be required to maintain stability. This would be achieved by either increasing trunk inclination or by increasing ITJ protraction angle (Andrada et al., 2014; Z. Wang et al., 2016). Therefore, the increase in ITJ angle may be compensatory to weak postural muscles and aid in maintaining balance during forward locomotion. While no study to date has investigated the role of temperature on posture muscle development, several chicken and turkey studies have demonstrated incubation temperature does affect muscle development (Clark et al., 2016; Collin et al., 2007; Loyau et al., 2013). In this case, failing to adequately compensate would result in a fall. However, this remains unclear because ITJ angle was only collected during successful strides. In any case, the large percentage of stride length variability observed in the cool thermal treatment and the small increase observed in the warm thermal treatment provide some initial evidence for this conclusion.

Nevertheless, early forms of quail locomotion are highly unsteady, relying on locally mediated spinal control circuitry which produces large amounts of variability, increasing the risk of falls (Hausdorff, 2007). For young terrestrial birds, running, albeit 
inefficiently, is the preferred gait for reduced energy expenditure with limited body control (Nudds et al., 2011). Stiff legged-running allows juvenile birds to benefit from passive spring biomechanical forces of the musculoskeletal system, thereby conserving energy and assisting with stabilization (Andrada et al, 2013; Biewener \& Daley, 2007; Nudds et al., 2011). Conversely, slower, more developmentally experienced movements are regulated by feedforward and feedback spinal-cortical mechanisms after the acquisition of extensive neuromuscular control and posture stability (Muir \& Chu, 2002; Muir et al., 1996). Our results support this pattern in that inexperienced locomoting hatchlings were more likely to fall at slower step cadences. An increase in stride variability further suggests a disruption in neuromuscular control.

Thermal fluctuations during the selected period of development for this study (E5 to E8) likely contributed to the observed perturbations in motor control. In the common quail, HH23 on the Hamburger and Hamilton (1951) staging series roughly begins on E5, which corresponds to the beginning of embryonic limb bud differentiation, primary muscle fibers having been laid in the trunk and secondary mesenchyme start to differentiate (Hendrickx \& Hanzlik, 1965; Stockdale, 1997). By E8, limb joints are distinct, all four toes are visible and webbing between toes is thinned and concave (Hendrickx \& Hanzlik, 1965). The final muscle, tendon, and cartilage pattern is present by E7.5 in chick embryos (Kardon, 1998) which corresponds roughly to stage 27 in a bobwhite quail embryo which ends on E8 (Hendrickx \& Hanzlik, 1965). Additionally, in Galliforme embryos, the hypothalamus begins to form on E6.5 and becomes functionally mature approximately at E13.5 (Debonne et al., 2008). In the current experiment, the mild experimental temperature changes during this sensitive window likely influenced 
endocrine processes modifying proliferation and differentiation of limb development (DuRant et al., 2014; DuRant et al., 2010; Hammond et al., 2007; Hopkins et al., 2011). Several studies across different species have highlighted the indirect effects of glucocorticoid signaling on kinase protein synthesis, satellite cell proliferation and differentiation, and muscle development (Belanto et al., 2010; Chin et al., 2009; Dong, Pan, \& Zhang, 2013; Nesan et al., 2012; X. Wang, Jia, Xiao, Jiao, \& Lin, 2015), which if dysregulated can have context dependent putative effects. Nesan and colleagues (2012) demonstrated the important role of glucocorticoids in embryo development using a receptor-knock-out fish model. The lack of glucocorticoid signaling decreased somite formation, and restricted optimal mesodermal development and myogenesis (Nesan et al., 2012). Conversely, excessive glucocorticoid signaling in mammals is known to have catabolic effects on muscles (Dong et al., 2013; Pereira \& Freire de Carvalho, 2011). A study by DuRant and colleagues (2014) with duck hatchlings found hatchlings incubated in cooler temperatures who had low body mass at postnatal day 9 (P9) also showed moderate increased corticosterone concentrations, the primary glucocorticoid in birds, at hatch and P9. Interestingly, cool incubated ducklings also showed poor motor performance (Hopkins et al., 2011), indicating a disruption in motor functioning. In the current study, low incubated birds showed a reduction in body mass and decreased motor coordination, which may indicate a disruption in glucocorticoid pathways.

Extensive research has outlined the importance of the hypothalamic-pituitarythyroid (HPT) axis in regulating avian basal temperature (for review see Debonne et al., 2008). These processes are tightly controlled by thyroid releasing hormone (TRH) (Debonne et al., 2008; Marsh \& Dawson, 1989; Olson, Vleck, \& Vleck, 2006). The up 
and down regulation of TRH is responsible for the increased or decreased production of thyroxine $\left(\mathrm{T}_{4}\right)$ and triiodothyronine $\left(\mathrm{T}_{3}\right)$ from the thyroid gland (Marsh \& Dawson, 1989). In young precocial birds, $T_{4}$ and $T_{3}$ mediates mitochondrial oxygen consumption, metabolic function (Marsh \& Dawson, 1989) and plays an important role in skeletal muscle phenotype formation (Deaton et al., 1997; Gardahaut et al., 1992; Salvatore et al., 2014). Further, several studies suggest thyroid hormones contribute to incubation period regulation (DuRant et al., 2014, 2010; Iqbal et al., 1990), body composition (Loyau et al., 2013), and social behavior (Gilbert et al., 2013; Lyall et al, 2017; Thompson et al., 2018). Additional research is required to determine if the observed motor disruptions in the current study can be attributed, at least in part, to thyroid regulated processes.

The present study provides evidence that relatively small modulations in typical prenatal incubation temperature are sufficient to affect early postnatal motor performance. Quail chicks in the low temperature condition showed greater variability in their stride length, larger ITJ angles, and increased fall frequency relative to controls, while contrary to our predictions, high temperature chicks showed an increase in falls and a decrease in TARS length. Additionally, the observed delay in hatching and decrease in body mass were consistent with previous studies. We conclude that prenatal temperature microclimates are important for optimal early postnatal motor development. 
Table 1

Descriptive summary of incubation and data inclusion criteria

\begin{tabular}{cc|ccc|cccc}
\hline Condition & Set & Candled & Hatched & Off-Cycle & Tested & Emerge & No Move & Total N \\
\hline Low & 75 & 14 & 53 & $15^{*}{ }^{2}, \mathrm{~b}$ & 38 & 7 & 3 & 28 \\
Control & 75 & 8 & 54 & $6_{\mathrm{a}}$ & 48 & 14 & 6 & 28 \\
High & 75 & 16 & 47 & $1_{\mathrm{a}}$ & 46 & 11 & 2 & 33 \\
Total & 225 & 38 & 154 & 22 & 132 & 32 & 11 & 89 \\
\hline \hline
\end{tabular}

Set=total number of eggs set for incubation, Candled=number of non-viable eggs during incubation, Hatched=number of chicks who exited the egg, Off-Cycle=number of chicks who hatched one day early (a) or one day late (b), Tested=number of chicks actually tested, No Emerge=number of chicks who failed to leave the emergence box, No Move=number of chicks who failed to move after leaving the emergences box, Total $N=$ number of chicks retained for data analysis. 
Table 2

Means (standard error) for spatiotemporal parameters

\begin{tabular}{c|c|c|c}
\hline & \multicolumn{3}{|c}{ Incubation Temperature Treatment } \\
Gait Measure & Low $\left(36.9^{\circ} \mathrm{C}\right)$ & High $\left(38.1^{\circ} \mathrm{C}\right)$ & Control $\left(37.5^{\circ}\right)$ \\
\hline ITJ Angle & $86.49(4.32)^{*}$ & $73.83(3.68)$ & $69.82(3.93)$ \\
ITJ CV & $17.34(1.1)$ & $18.94(0.94)$ & $19.10(1)$ \\
Stride Length & $3.11(.11)$ & $3.40(.09)$ & $3.13(.10)$ \\
Stride Length CV & $27.30(1.36)^{*}$ & $24.74(1.16)$ & $21.87(1.24)$ \\
Step Width & $.63(.05)$ & $.59(.04)$ & $.66(.04)$ \\
Step Width CV & $39.96(2.69)$ & $42.85(2.29)$ & $35.96(2.45)$ \\
Stance Width & $1.42(.053)$ & $1.42(.045)$ & $1.35(.049)$ \\
Stance Width & $24.37(1.16)$ & $26.40(.99)$ & $24.04(1.06)$ \\
CV & & & \\
Velocity & $7.62(.62)$ & $6.62(.48)$ & $5.91(.59)$ \\
\hline \hline
\end{tabular}

ITJ angle=degrees, Stride Length=cm, Step Width=cm, Stance Width=cm,

$C V=$ Coefficient of variation, a percentage of variability for each kinematic parameter, Velocity $=\mathrm{cm} / \mathrm{s}$. Results reflect comparisons to the control incubation treatment after statistically accounting for the confounding influence of body composition, velocity and step cadence. *Bonferroni corrected $p<0.015$ 


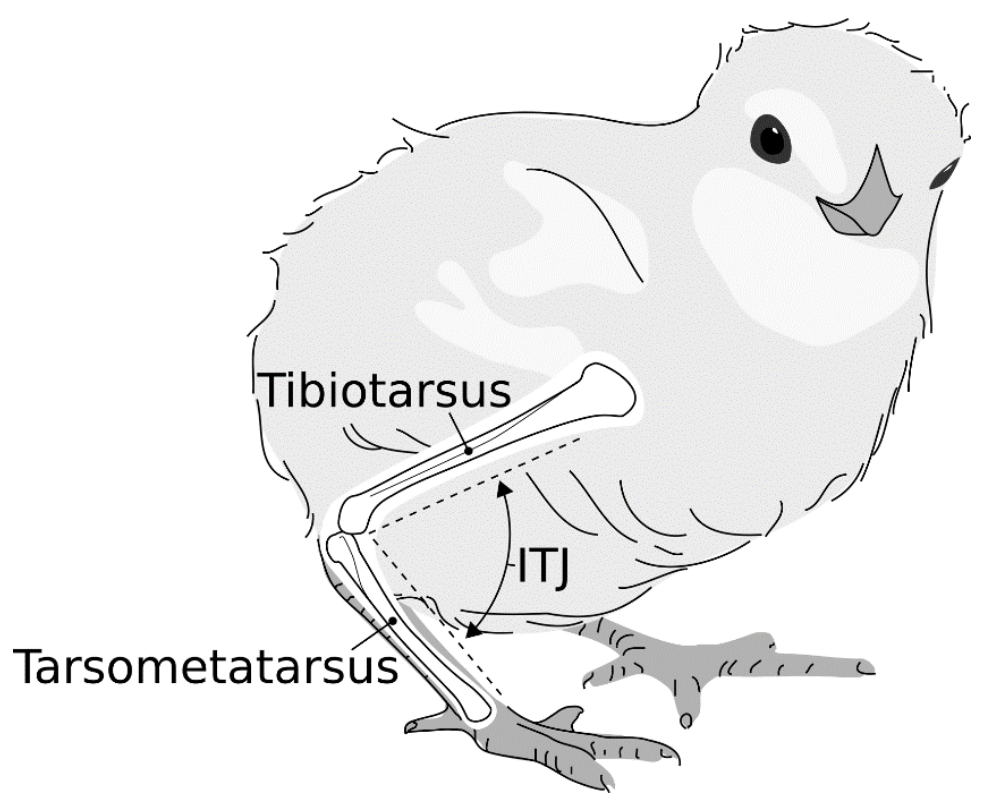

Figure 1

Illustrated measurements of TARS and ITJ angle in a neonatal quail chick. TARS length was measured from the intertarsal notch (ITJ junction) to the junction of the first digit. The protraction ITJ angle was calculated at initial touchdown during the gait cycle by drawing two reference lines depicted by the dashed lines. The first line followed the TARS bone and the second began at the intertarsal notch up the lateral side of the body, approximating the position of the tibiotarsus.

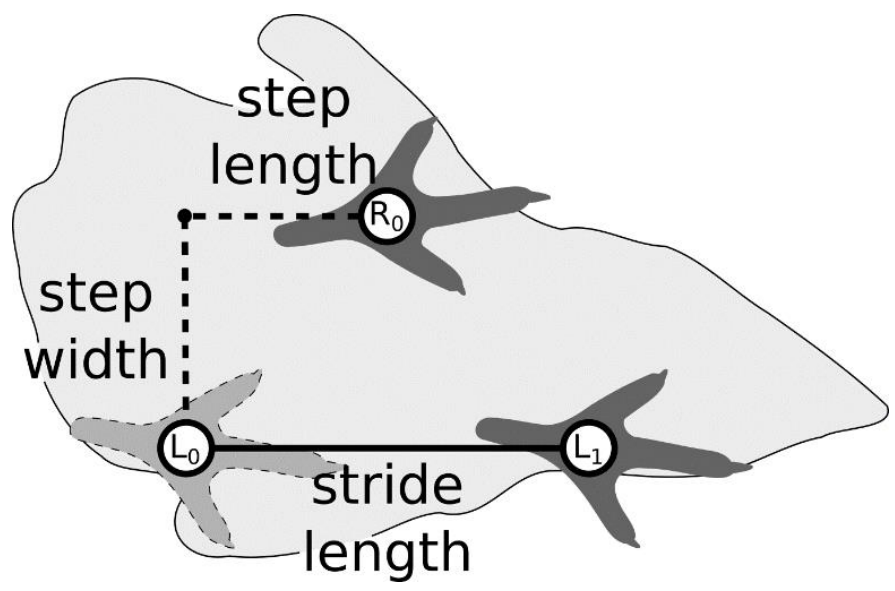

Figure 2

Illustrated spatiotemporal measurements from a posterior view of a neonatal chick. Stride length was defined as the distance between consecutive foot strikes of the same foot (i.e. $\mathrm{L}_{1}-\mathrm{L}_{0}$ ). Step width was equal to the distance between consecutive left and right foot strikes in the mediolateral direction. Stance width was the distance between the right and left foot during rest (i.e $\mathrm{R}_{0}$ to $\mathrm{L}_{1}$ after $\sim 3$ seconds of no movement). 


\title{
Body Composition
}

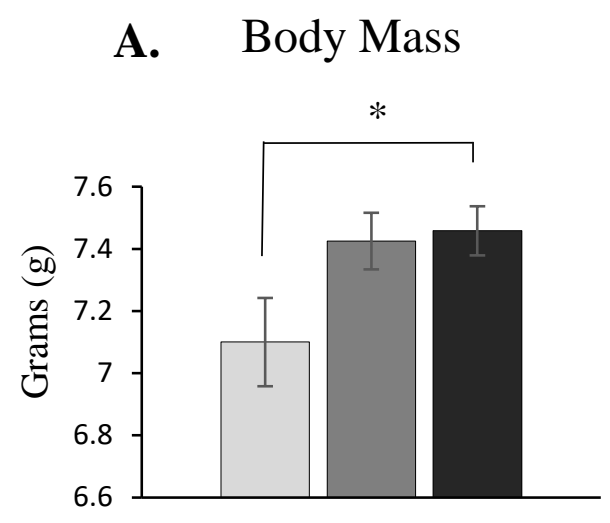

\author{
B. Tarsometatarsus Length
}

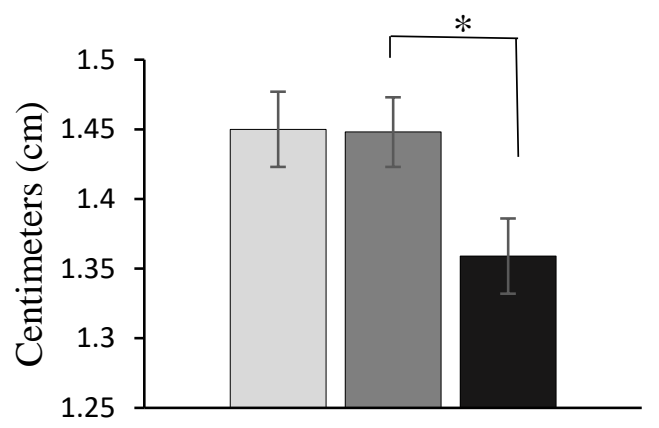

Temperature Treatment

$\square$ Low $\square$ Control $\square$ High

Figure 3

Body Composition measured 24-hours after hatching. Chicks experienced three prenatal temperature conditions: low $\left(36.9^{\circ} \mathrm{C}\right)$ illustrated in light gray, control $\left(37.5^{\circ} \mathrm{C}\right)$ illustrated in dark gray, and high $\left(38.1^{\circ} \mathrm{C}\right)$ illustrated in black. A: Body mass measured in grams for each temperature condition. B: Tarsometatarsus length measured in centimeters for each temperature condition. Bars show means; vertical lines depict SEM. *Bonferroni corrected $\mathrm{p}<.015$ 
Falls vs Step Cadence

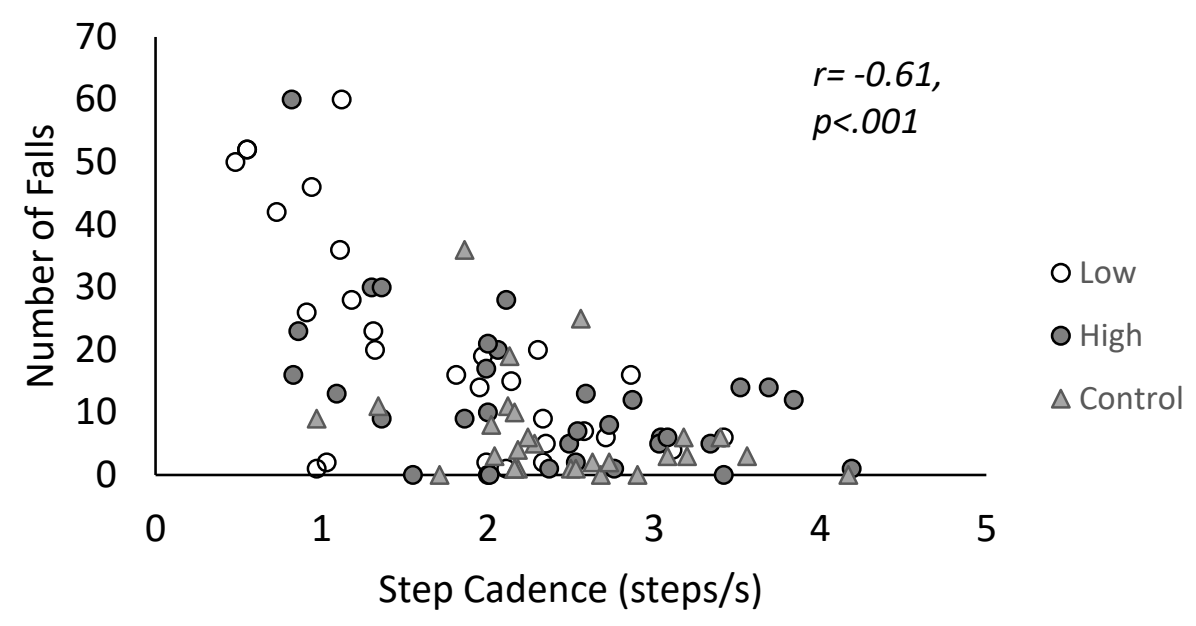

Figure 4

The correlation between fall frequency and step cadence (steps per second of activity) for each hatchling per incubation temperature treatment. Each dot represents a single bird from either low $\left(36.9^{\circ} \mathrm{C}\right.$ open circle), high $\left(38.1^{\circ} \mathrm{C}\right.$ closed circle), or control $\left(37.5^{\circ} \mathrm{C}\right.$ closed triangle). 


\section{Behavior Measurements}

A. Step Cadence

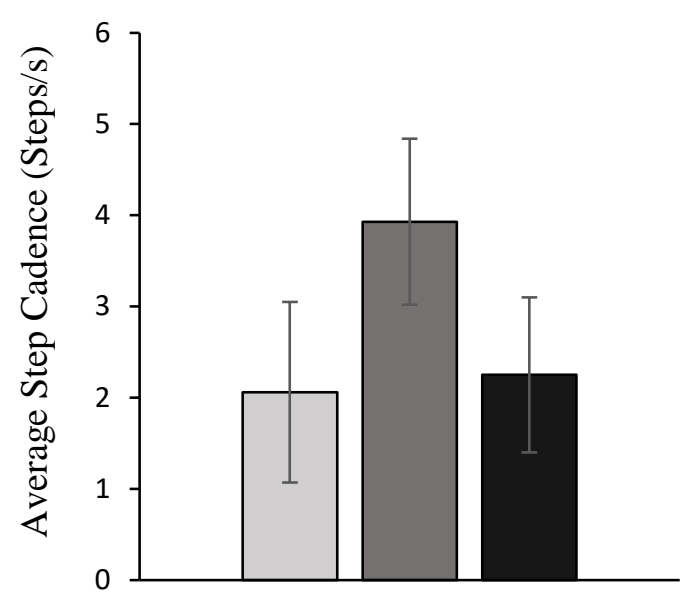

B. Fall Frequency

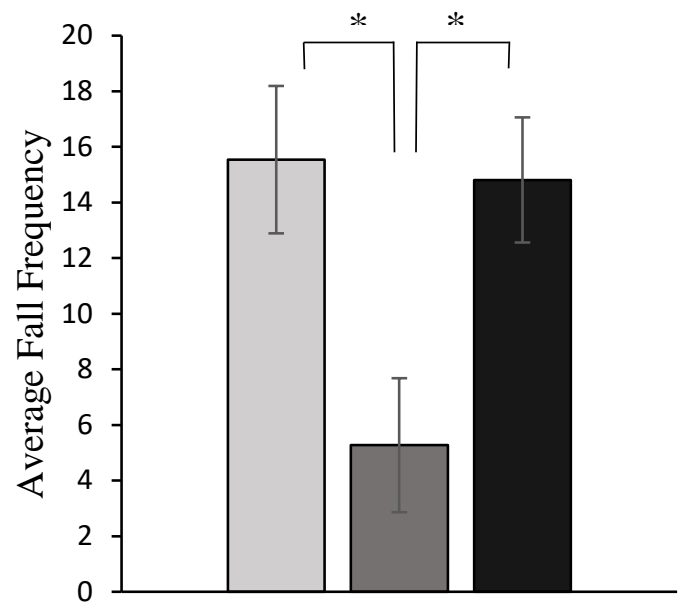

Temperature Treatment

$\square$ Low $\square$ Contro $\square$ High

\section{Figure 5}

Behavioral measurements for each temperature treatment measured at 24-hours after hatching. All values account for differences in body composition, and velocity. Chicks experienced four days of one of three prenatal temperatures during the treatment window (E5 -E8): low $\left(36.9^{\circ} \mathrm{C}\right)$ illustrated in light gray, control $\left(37.5^{\circ} \mathrm{C}\right)$ illustrated in dark gray, and high $\left(38.1^{\circ} \mathrm{C}\right)$ illustrated in black. A: Step cadence reflects the average number of steps taken per second of activity. B: Falls represents the number of falls per video after controlling for step cadence. Bars show means; vertical lines depict SE. *Bonferroni corrected $\mathrm{p}<.015$ 


\title{
Study 2
}

\author{
EFFECTS OF INCREASED AND DECREASED EARLY INCUBATION \\ MOVEMENT ON NEONATAL PRECOCIAL BIRDS' LOCOMOTOR BEHAVIOR
}

This manuscript was submitted for publication to the Journal of Developmental

Psychobiology August 2019

Starlie C. Belnap, M.S. ${ }^{1 *}$, Edgar R. Vieira, PT, MSc, $\mathrm{PhD}^{2}$, Robert Lickliter, $\mathrm{PhD}^{1}$

${ }^{1}$ Department of Psychology, Florida International University,

${ }^{2}$ Depeartment of Physical Therapy, Florida International University

*Corresponding author: Starlie Belnap, sbelnap@ fiu.edu, 305-348-1230, Department of Psychology, Florida International University, Miami, FL 33199.

\section{Acknowledgements}

This research was supported by NSF grant BCS 1525371 awarded to RL. We thank John P. Currea, M.S. for assistance with software development. We also thank all the student researchers who assisted with this project. 


\begin{abstract}
Comparative animal studies have aided in understanding how prenatal mechanical forces and vestibular and tactile sensory experiences resulting from maternal activity facilitate or interfere with growth and perceptual development. However, there is a paucity of information on how prenatal movement experience influences postnatal motor performance. In the present study we used an avian model, the bobwhite quail, to evaluate the effects of decreased and increased prenatal movement on growth and postnatal motor performance in $24 \mathrm{hr}$ quail neonates. We compared all metrics to chicks who received industry standard normal movement experience. Experimental movement treatment began on embryonic day eight (E8) and ended on E14. Motor performance and body mass and tarsometatarsus length (indicators of growth) were evaluated 24-hours after hatching. As anticipated, decreased movement exposure during incubation delayed hatching, reduced body mass, and decreased motor performance relative to normal movement exposure. Contrary to predictions, increased movement exposure also showed delayed motor performance and a reduced body mass compared to normal movement exposure, but increased movement exposure did not delay hatching. Further, limb length did not vary by treatment condition. Results confirmed that gait analysis can detect subtle changes in motor performance, previously not detected by other motor tasks. The study provides the first evidence that early prenatal movement can interfere with typical motor development and indicates that maternally regulated movement contributes to postnatal motor performance, independent of maternal metabolic processes.
\end{abstract} Keywords: Motor performance, prenatal, vestibular, tactile, sensorimotor, development 


\section{Introduction}

During prenatal development there are many maternal factors which contribute to the health and wellbeing of the fetus. Some of these factors have lasting epigenetic consequences which can extend into adulthood (Wu, Bazer, Cudd, Meininger, \& Spencer, 2004). For example, nutrition during the first few weeks of gestation plays an important role for placental growth and vascularization, which are necessary for oxygenation and nutrient transmission throughout gestation (Han \& Carter, 2001; Wu et al., 2004).

Humans and animals with restricted placental growth experience restricted fetal growth which corresponds to low weight at birth or hatch (Dwyer, Madgwick, Ward, \& Stickland, 1995; Godfrey, Robinson, Barker, Osmond, \& Cox, 1996; Redmer, Wallace, \& Reynolds, 2004; Robinson et al., 1997). Maternal diets low in protein and high in carbohydrates have been associated with small placental surface area and low birthweight (Godfrey et al., 1996). In addition to nutritional factors, maternal exercise and activity modulates placental vascularization and fetal growth (Clapp, 2006; Clapp \& Rizk, 1992; Mangwiro et al., 2018; Rosa et al., 2011). Whereas most research confirms that mild to moderate exercise is beneficial (Clapp \& Rizk, 1992; Rosa et al., 2011), an extreme sedentary lifestyle paired with obesity (Higgins, Greenwood, Wareing, Sibley, \& Mills, 2011) and extremely strenuous exercise (Oliveira, Fileto, \& Melis, 2004; Salvesen, Hem, \& Sundgot-Borgen, 2012; Szymanski \& Satin, 2012) appears to compromise fetal wellbeing. However, in mammalian research it is difficult to separate maternal movement and nutrition because of their metabolic dependency, making it difficult to fully understand the role of movement on fetal growth. Further, very few studies have investigated the effects of prenatal movement on other neurodevelopmental outcomes. 
Precocial birds (e.i., chicken and quail) are a particularly useful animal model for research because the maternal hen only influences the nutrition available to the embryo during egg formation. Like mammals, avian embryos benefit from prenatal movement experience (Deeming, 2009; Robertson, 1961; Wilson, 1991), which also facilitates vascular development (Deeming, 1989) and embryonic growth (Deeming, 2009; Lilienthal, Drotleff, \& Ternes, 2015; Tullett \& Deeming, 1987). Further, the amount of movement an embryo receives, under natural conditions, is regulated by the behavior of the nesting hen (Olsen, 1930; Orcutt \& Orcutt, 1976). Olsen (1930) reported that wild chickens rotate their eggs approximately every 15 minutes during early incubation by nudging eggs with their beak or feet. Similar behavior was observed in pheasants and quail (Orcutt \& Orcutt, 1976). Additionally, there are several important neurodevelopmental similarities in the organization of motor and sensory systems (Gottlieb, 1971; Muir, 2000; Oppenheim, 1972), and because they are precocial, their sensory and motor systems are fully functional at hatching, making it possible to evaluate motor function with little confounding effects from postnatal experience. Unlike, mammalian research, with precocial birds we can precisely control embryonic developmental age as well as prenatal experiential history, since incubation is possible within the lab. By leveraging these unique properties, it is possible to isolate the effects of prenatal movement exposure and investigate its role in postnatal motor development.

Even though precocial birds are capable of locomotion shortly after hatching, they still require several days of experience to develop an efficient oscillating gait pattern to minimize energy expenditure while maximizing mobility (Muir \& Chu, 2002; Muir et al., 1996). Inexperienced young chicks prefer a fast gait with short strides which reduces 
energy expenditure and aides with stabilization and balance, while more experienced chicks prefer a slower gait with longer strides (Muir et al., 1996; Nudds et al., 2011). Research has shown these patterns can be altered by prenatal sensory experience (Belnap, Currea, \& Lickliter, 2019; Belnap \& Lickliter, 2017) and by postnatal locomotor deprivation (Muir \& Chu, 2002). Young chickens who were postnatally deprived of locomotor experience continued to show an immature gait pattern, with an increase in step cadence and a decrease in stride length compared to age matched controls (Muir \& Chu, 2002). Likewise quail hatchlings with limited prenatal light experience showed greater instability and fell more frequently compared to control hatchlings (Belnap \& Lickliter, 2017).

Prenatal movement experience through maternal egg turning exposes the growing embryos to mechanical stress and sensory experience. During egg turning, the fluid within the egg flows and presses against the amnion, which is also filled with protective amniotic fluid, gently shifting the embryo. Maternal egg movements not only subjects the structural components of the egg to mechanical forces, but they also provide the growing embryo with tactile and vestibular sensory stimulation. Detailed research provides some understanding of how the mechanical stress of egg turning influences biological processes and structure formation (Baggott, Deeming, \& Latter, 2008; Deeming, 1989, 2009; Fermin et al., 1996; Lychakov et al., 1993; Wilson, 1991), but very few studies have evaluated how the sensory (vestibular and tactile) stimulation of egg turning influences perceptual processes and behavior (Carlsen \& Lickliter, 1999; Honeycutt \& Lickliter, 2003). From the few studies that have investigated the role of movement on perception, we know that increased prenatal vestibular and tactile experience can alter the 
sensory responsiveness of other developing sensory modalities (Carlsen \& Lickliter, 1999); however, the nature of its effect depends on the developmental timing of when the exposure occurs (Honeycutt \& Lickliter, 2003). These two studies highlight the importance vestibular and tactile experience and its timing for typical development. However, to date no study has investigated the effects of egg turning on gait performance, a highly complex motor skill which relies on tactile and vestibular feedback.

We know from surgical and pharmacological sensory deprivation studies in birds and mammals that vestibular and tactile sensory experience are also important for optimal motor performance (Geisler \& Gramsbergen, 1998; Ronca, Fritzsch, Alberts, \& Bruce, 2000). The vestibular system aides in the stabilization of eye, neck, body position, and spatial orientation in response to changes in the head (Dickman \& Lim, 2004; Geisler \& Gramsbergen, 1998; Gottesman-Davis, 2011; Kenyon, Kerschmann, \& Silbergleit, 1988). In birds, a loss of vestibular feedback results in a loss of equilibrium, balance, and postural control, which effectively inhibits goal oriented locomotion (Dickman \& Lim, 2004; Kenyon et al., 1988). Research with preterm infants who receive unusually early augmented vestibular and tactile experience (Field, Diego, \& Hernandez-Reif, 2010; Nelson et al., 2001) and animal neural injury studies (Maldonado, Allred, Felthauser, \& Jones, 2008; Muir \& Steeves, 1995) indicate that sensorimotor training, including touch and vestibular sensation, can aide in motor recovery. However, the role of prenatal movement experience on early motor development is not well understood. The current experiment was designed to address this knowledge gap. 
In the current study we examined if gait performance, body composition, and incubation length in $24 \mathrm{hr}$ bobwhite quail hatchlings would be influenced by prenatal movement experience. Since previous avian research has documented the negative effects of the absence of egg turning experience (Robertson, 1961; H. R. Wilson, 1991), our study used a decreased prenatal movement treatment, reducing egg-turning by $50 \%$ during incubation, to evaluate how reduced vestibular and tactile experience could influence hatching, growth, and postnatal motor performance. We predicted that decreased prenatal movement would delay development by increasing incubation length, decreasing body weight and tarsometatarsus length (measurements of body composition), and delay motor performance when compared to chicks who received industry standard normal movement stimulation of one turn every two hours. In contrast to research on decreased levels of turning, research on increased levels of turning have found little to no effect on hatchability, nor were there any reported difficulties in motor performance (Carlsen \& Lickliter, 1999; Honeycutt \& Lickliter, 2003; Robertson, 1961). Given thier findings, we predicted chicks incubated under conditions of increased prenatal movement would show typical developmental patterns, by hatching within the typical incubation period, have similar body composition as controls, and show accelerated motor development compared to normal prenatal movement controls. Further we anticipated that gait analysis would accurately predict movement treatment.

\section{Methods}

\section{Incubation \& Housing}

Two hundred and fifty fertilized bobwhite quail eggs were obtained from a commercial game bird supplier (Strickland) over the course of ten weeks. Each week 25 
eggs were randomly selected and placed into one of three digital temperature and humidity-controlled incubators (King R-com) and placed upon an automatic turning cradle until 75 eggs were assigned to each of the experimental treatment conditions. The standard cradle rotated the entire incubator $45^{\circ}$ per turn cycle at a rate of 30 s every $2 \mathrm{hrs}$ providing embryos with vestibular and tactile sensory experience during incubation. The day fertile eggs were placed in an incubator was recorded as day zero and eggs remained in their assigned incubator until embryonic day 21 (E21) when they were removed from the turning cradle until day of hatching (E23). The incubators were maintained at 65\% $\pm 5 \%$ humidity and $37.5^{\circ} \mathrm{C} \pm 0.2^{\circ} \mathrm{C}$ on a $12 \mathrm{hr}$ light/dark cycle within a four-chamber stimulation cabinet (Belnap et al., 2017, 2019) throughout the entire incubation period. To ensure the stability of temperature and humidity, the incubators were monitored every four hours throughout the day.

Starting on embryonic day E8 embryos received one of three movement treatments, increased, decreased, or normal for a seven-day period. The selected manipulation period overlaps with the onset of vestibular (Ginzberg \& Gilula, 1980) and tactile (Gottlieb, 1968) sensory functioning, and the onset rhythmic embryonic movement (Hamburger \& Balaban, 1963), after accounting for small variations in the timing between chicken and quail developmental stages (Hendrickx \& Hanzlik, 1965). The increased movement condition received 10 times the normal amount of movement experience following the same pattern described by Carlsen and Lickliter (1999). Eggs turned at a rate of one turn per 30s for 10mins every $4 \mathrm{hrs}$ resulted in a total of 420 mins of vestibular and tactile experience during the seven-day manipulation period. Because of the possible negative effects of a severe reduction in movement experience (Deeming, 
1989; Robertson, 1961), embryos in the decreased movement condition received only $50 \%$ less the normal amount of movement experience. In the reduced movement treatment, eggs turned at a rate of one turn per 30 s every 4 hrs resulted in a total $21 \mathrm{mins}$ of vestibular and tactile experiences during the seven-day manipulation period. The normal movement condition received standard incubation care resulting in a total of 42 mins of movement experience during the seven-day manipulation period. Modified turning experience was provided through a specially customized turning cradle (King Rcom) which was controlled by a customizable automation program designed for Samsung Smartthings controllers.

On day 15 , the eggs were candled to identify infertile eggs and non-developed embryos (see table 1). Upon hatching, chicks were transferred by movement condition and day of hatch (E22, E23, and E24) into clear plastic rearing tubs in clutches of 12-15 to simulate naturally occurring brooding conditions. Chicks who hatched on day 22 were excluded from testing. Chicks' distal phalanges were marked with red paint (Revlon quick drying nail polish) for individual identification (traditional leg bands were avoided to prevent confounds during motor testing). Tubs were maintained in a sound-attenuated rearing room at $30^{\circ} \mathrm{C}$ on a $12 \mathrm{hr}$ light/dark cycle. Food and water were available ad libitum. All testing and animal care followed procedures and protocols approved by the Institutional Animal Care and Use Committee \#200826.

\section{Gait Task and Body Composition Measures}

Chick motor coordination was evaluated using a 5-minute gait task one day following hatch. The 5-minute gait task consisted of a 1-minute acclimation period within 
a dark emergence box $(10 \mathrm{~cm} \times 10 \mathrm{~cm} \times 10 \mathrm{~cm})$ followed by a 4 -minute free roam exploration period within a rectangular Plexiglass runway. One hour prior to testing, square reflective tape $(.5 \times .5 \mathrm{~cm})$ was placed in the center of the right and left tarsal pads to enhance visibility of foot placement during video scoring. To decrease handling effects and stress, chicks were transported in an opaque plastic container to the testing runway. Only chicks who emerged from the box were evaluated for motor coordination (see Table 1). Following testing, chicks were immediately returned to the rearing room, where they were weighed and the right tarsometatarsus (TARS) length was measured. The TARS length was measured as the distance between the notch at the intertarsal joint (ITJ) and the juncture of the TARS and the first digit.

The entire 5-minute testing session occurred within a Plexiglas rectangle runway outfitted with a dark removable lid $(95 \mathrm{~cm}$ long x $13 \mathrm{~cm}$ wide $\mathrm{x} 20 \mathrm{~cm}$ tall) raised approximately one meter above the floor. The Plexiglas rectangle runway sat within a wooden support frame housed in a temperature-controlled room $\left(30^{\circ} \mathrm{C} \pm 1^{\circ} \mathrm{C}\right)$. A fake black foamboard floor suspended approximately $40 \mathrm{~cm}$ below the runway was fitted around a black USB camera (60fps 1230x 720dpi) which was connected to a computer located in an adjacent room. The floor hid the inferior camera from the hatchlings view and provided the visual effect of a solid floor. A second white USB camera was affixed on the white wall outside the runway to provide a lateral view and was connected to the same computer. The start area contained the emergence box which remotely opened after the 1-minute acclimation period. A speaker isolated from the main runway located opposite from the start area calibrated at $65 \mathrm{~dB}$ continuously played a bobwhite quail maternal call to motivate hatchlings to cross the runway during the test period. The lateral 
and inferior camera views were recorded using Kinovea, an open source kinematic software program.

\section{Gait parameters}

Inferior videos were behaviorally scored using Datavyu (Datavyu Team, 2014), a quantitative event-recording behavioral program, for frequency of forward steps and falls. Steps were defined as controlled forward movement, (walk, ground run, or aerial run) relative to orientation of the chick's head (Belnap \& Lickliter, 2017). The definition excluded lateral movement (i.e. side steps used in turning), movement with no distance traveled (i.e. shifting of center of gravity from foot to foot), and backwards movement (relative to chick orientation not the speaker area). Chicks were scored as falling, a measurement of instability, when their upper thorax or head contacted with the runway's floor. Chicks in a crouched position, knees and hip joints flexed with the thorax resting on the ground, were not scored as falling. Step cadence was defined as steps per second of active movement.

Spatiotemporal parameters and body kinematics including stride length, step width, stance width, velocity, and ITJ were measured using Kinovea. Inferior videos were evaluated for each parameter on separate frame by frame viewing passes until 20 usable measurements of each parameter were collected. The supporting wooden frame was used as a reference for measurement calibration. Stride length, step width, and stance width were scored using the inferior view. Velocity and ITJ angles were scored using the lateral camera view. Chicks who failed to emerge from the start box or who failed to produce 
more than 10 useable data points because of lack of movement were excluded from analysis (see Table 1).

For stride length and step width, only successfully completed gait cycles were used to calculated averages. Measurements for stride length were acquired at touch down for each limb throughout the entire video. Stance width, distance between left and right limb during standing, was acquired during moments of rest. Rest was defined as $180(\sim 3$ seconds) frames of non-movement, including forward movement, falls, jumps, and other forms of instability.

Gait speed and ITJ angles were evaluated using the lateral view and calibrated using the wood support frame. The ITJ angle was measured at initial touchdown of the gait cycle to capture maximum leg extension. Measurements occurred when the hatchling's locomotion was perpendicular to the camera, providing a clear profile view without video distortion of the right limb. Approximately 20 useable frames were acquired throughout the video for each bird with a minimum of 10 useable frames were needed to be included in the analysis. The ITJ angles were estimated by drawing two digital reference lines within Kinovea. The first line began at the juncture of the TARS and the third digit and followed the length of the TARS. The second line began at the notch of the ITJ and extended up the lateral side of the body approximating the position of the tibiotarsus (see Belnap, Currea, \& Lickliter, 2019). The base of the digital protractor tool within Kinovea was placed on the first reference line and then extended to the second reference line producing the estimated protraction ITJ angle per stride for the right limb. Gait speed was calculated only during bouts of forward locomotion using the following formula $\frac{x_{2}-x_{1}}{t_{2}-t_{1}}$, where $\mathrm{x}$ represents the bird's position and t represents time for 
each frame within a bout. Locomoting bouts were scored manually by starting and stopping the tracking feature within Kinovea. A bout initiated at toe off and ended at touchdown of a sequence of forward locomotion. In the event a bird became unstable during the sequence, the locomoting bout concluded the gait cycle prior to the instability. Gait speed reflected the change in position over the change in time averaged across all locomotion bouts.

Four observers were assigned to each performance measure. Prior to scoring, observers were trained until inter- and intra-rater reliability of $>85 \%$ was achieved. Upon completion of scoring, roughly $10 \%$ of videos were randomly selected to be rescored to verify accuracy for each measure. Observers were blind to the movement condition and relied upon individual bird identification markings, which were void of treatment assignment, to report results.

\section{Data Analysis}

Because of nonnormality, all behavioral (step cadence and fall frequency) and spatiotemporal parameters were bootstrapped with replacement for 2,000 iterations. Right and left limb measurements for stride length were evaluated using paired sample t-tests. Chi-square likelihood tests and simple binomial outcome tests were used to characterize the influence of movement on developmental age and gait task emergence. Differences in body composition and gait speed between movement conditions were evaluated using one-way analysis of variance (ANOVA) and were controlled for in subsequent analysis. Spatiotemporal parameters and behavior were analyzed using multivariate analyses of variance (MANOVA; three movement conditions on 4 gait measures and two behavior 
measures) with body composition, gait speed, and developmental age listed as covariates. The MANOVA analysis accounts for potential correlations between dependent variables allowing for differences to be assessed without increasing type I error (Warne, 2014). Significant gait variables were further analyzed using univariate Bonferroni post hoc analyses with alpha adjustment set at p-values 0.015 . Since the frequency of falls may be attributed to differences in activity between movement treatments, the relationship between step cadence and fall frequency was evaluated using Pearson correlation. If the preceding relationship was significant then step cadence was included as an additional covariate in post hoc analyses for falls. To evaluate the sensitivity, and odds ratios of gait parameters and body composition on predicting movement treatment condition a multinomial logistic regression was performed. To aide with interpretation all values were mean centered. model fit was assessed using the likelihood ratio test. All statistical tests were conducted using SPSS v.22. Statistical significance was set at alpha .05. 95\% confidence intervals and effect sizes were calculated and interpreted using guidelines suggested by Durlak (2009).

\section{Results}

\section{Hatch Description}

A total of 32 eggs were removed during the candling process with no significant differences between conditions (see Table 1). Because of health concerns and physical leg deformities a total of 5 chicks from the decreased movement treatment were not tested. Three of these chicks hatched on E24 and two hatched on E23. Chi-square comparisons of developmental age by movement treatment showed significant differences $\left(X^{2} \mathrm{df}(4)=44.07 \mathrm{p}<0.001\right)$. A total of 183 chicks hatched with 16 chicks 
hatching early, 20 chicks hatching late, and 147 chicks hatching on-time. Six of the early hatching chicks belonged to normal movement treatment while the remaining ten chicks belonged to the increased movement treatment. All the late hatching chicks belonged to the decreased movement treatment. However, the distribution of chicks who hatched on E23 did not vary by movement condition. When evaluating the likelihood of emerging during the gait task, chi-square results revealed chicks behaved similarly across all movement treatments $\left(X^{2} \mathrm{df}(2)=3.064 \mathrm{p}=0.22\right)$. The failure to move after exiting the start box was also similar across movement treatments $\left(\mathrm{X}^{2} \mathrm{df}(2)=.44 \mathrm{p}=0.8\right)$. Even though significant differences were not detected, the decreased movement treatment condition had a slightly higher number of chicks excluded from analysis and a slightly lower hatch rate which required an additional batch of eggs be set to obtain sufficient power for statistical analysis.

\section{Body Composition \& Developmental Age}

Evaluations of body mass found significant differences between movement treatments $\left(\mathrm{F}(2,90)=15.753, \mathrm{p}<0.001, \eta^{2}=.26\right)$ and developmental age $(\mathrm{F}(1,90)=4.58$, $\left.\mathrm{p}<0.05, \eta^{2}=.05\right)$. Hatchlings incubated with normal movement stimulation $(\mathrm{M}=7.43$, $\mathrm{SE}=.13,95 \% \mathrm{CI}[7.25-7.60])$ weighed significantly more compared to chicks incubated with increased prenatal movement stimulation $(\mathrm{M}=6.46, \mathrm{SE}=.12,95 \% \mathrm{CI}[6.16-6.73])$ and chicks incubated with decreased prenatal movement stimulation $(\mathrm{M}=6.74, \mathrm{SE}=.14$, 95\%CI [6.5 - 6.98]). Further the weight between increased movement and decreased movement treatment conditions also significantly varied (see Figure 1). Chicks who hatched on E23 (M=6.97, SE=.07, 95\%CI [6.82 - 7.11] weighed significantly more than 
chicks who hatched on E24 (M=6.44, SE=.24, 95\%CI [6.04 -6.88]). However significant differences were not found for TARS length for developmental age $(F(1,90)=.88$, $\left.\mathrm{p}=0.352, \eta^{2}=.01\right)$ or movement treatment $\left(\mathrm{F}(2,90)=2.80, \mathrm{p}=0.07 \eta^{2}=.06\right)$.

\section{Gait Parameters}

Evaluations of gait speed found significant differences between movement treatment $\left(\mathrm{F}(2,89)=11.194, \mathrm{p}<0.001, \eta^{2}=.20\right)$, but not developmental age $(\mathrm{F}(1,89)=1.02$, $\left.\mathrm{p}=0.32, \eta^{2}=.01\right)$. Chicks in the normal prenatal movement treatment $(\mathrm{M}=5.91, \mathrm{SE}=.62$, $95 \% \mathrm{CI}[4.83-7.20])$ walked slower compared to chicks in the increased prenatal movement treatment $(\mathrm{M}=9.79, \mathrm{SD}=.59,95 \% \mathrm{CI}[8.71-10.96])$ and the decreased prenatal movement treatment $(\mathrm{M}=8.28, \mathrm{SE}=.66,95 \% \mathrm{CI}[7.0-9.60])$. However, chicks in the increased movement and decreased movement treatments did not differ (see Table 2). Because gait speed may influence step cadence and fall frequency, gait speed continued to be listed as a covariate in subsequent analysis. Pearson correlation test between step cadence and falls revealed a non-significant negative relationship ( $\mathrm{r}=-.07$, $\mathrm{p}=.53,95 \% \mathrm{CI}[-.17-.04])$, indicating fall rate was not related to the number of steps taken when active. A paired t-test comparison between right and left limb stride length for each movement treatment revealed no significant differences between limbs for the normal movement and increased movement treatments. However, there was a significant difference in stride length between the right and left limb for the decreased prenatal movement treatment $(\mathrm{t}(33)=-3.64, \mathrm{p}<.001)$. The left limb $(\mathrm{M}=2.65, \mathrm{SE}=.44)$ on average had shorter strides compared to the right limb ( $\mathrm{M}=2.82, \mathrm{SE}=.58)$. Subsequent analysis included only the right limb values since TARS length and ITJ angles were only acquired for the right limb. 
We ran a MANOVA with movement treatment as the independent variable and the gait parameters as the dependent measures with gait speed, body composition, and developmental age as covariates. Of the covariates included in the model, only gait speed significantly contributed to the overall model (Pillai's Trace $=0.37 \mathrm{~F}(6,80)=7.87, \mathrm{p}<0.001$, $\left.\eta_{\mathrm{p}}{ }^{2}=0.37\right)$. Gait speed was significantly related to stride length $(\mathrm{F}(1,85)=21.72, \mathrm{p}<0.001$, $\left.\eta_{\mathrm{p}}{ }^{2}=.20\right)$, stance width $\left(\mathrm{F}(1,85)=4.33, \mathrm{p}<0.05, \eta_{\mathrm{p}}{ }^{2}=.05\right)$, and cadence $(\mathrm{F}(1,85)=22.22$, $\left.\mathrm{p}<0.001, \mathrm{\eta}_{\mathrm{p}}^{2}=.21\right)$. As speed increased stride length, step cadence and the BoS increased. After controlling for gait speed, the movement treatment continued to contributed to the model (Pillai's Trace $\left.=0.55 \mathrm{~F}(12,162)=5.15, \mathrm{p}<0.001, \eta_{\mathrm{p}}{ }^{2}=0.28\right)$. Movement treatment significantly influenced stride length $\left(\mathrm{F}(2,85)=8.57, \mathrm{p}<.001, \mathrm{\eta}_{\mathrm{p}}{ }^{2}=0.17\right)$ and step width $\left(\mathrm{F}(2,85)=11.07, \mathrm{p}<.001, \mathrm{\eta}_{\mathrm{p}}{ }^{2}=0.21\right)$ as shown in Table 2. However, movement treatment failed to significantly influence fall rate $\left(\mathrm{F}(2,85)=11.07, \mathrm{p}<.001, \eta_{\mathrm{p}}{ }^{2}=0.21\right)$ or step cadence $\left(\mathrm{F}(2,85)=11.07, \mathrm{p}<.001, \mathrm{\eta}_{\mathrm{p}}{ }^{2}=0.21\right)$ as anticipated. Chicks who experienced decreased prenatal movement $(\mathrm{M}=2.75, \mathrm{SE}=. .09,95 \% \mathrm{CI}[2.57-2.94])$ and increased prenatal movement $(\mathrm{M}=2.62, \mathrm{SE}=.11,95 \% \mathrm{CI}[2.41-2.83])$ during incubation took significantly smaller steps compared to chicks who experienced normal prenatal movement $(\mathrm{M}=3.26, \mathrm{SE}=.11,95 \% \mathrm{CI}[3.04-3.47])$. Additionally, increase movement treated chicks took narrower steps $(\mathrm{M}=.51, \mathrm{SE}=.04,95 \% \mathrm{CI}[.44-.57])$ when compared to decrease movement $(\mathrm{M}=.73, \mathrm{SE}=.03,95 \% \mathrm{CI}[.67$ - .79]) and normal movement $(\mathrm{M}=.66$, $\mathrm{SE}=. .04,95 \% \mathrm{CI}[.58-.73])$ treated chicks. However, decreased movement treated chicks did not differ significantly from normal movement treated chicks. No significant differences were discovered for other gait parameters (see Table 2). 
The multinomial logistic regression model which used gait parameters and body composition to predict movement treatment was significant $\left(\mathrm{R}^{2}\right.$ mcfadden $=.51$, $\left.\chi^{2}(18)=102.16, \mathrm{p}<.001\right)$. Gait and body composition accurately predicted vestibular treatment condition $79 \%$ of the time, with normal movement treated chicks being accurately predicted $80 \%$ of the time, increase movement treated chicks being accurately predicted $74 \%$ of the time, and decreased movement treated chicks being accurately predicted $86 \%$ of the time. This predictive model reflects a $49 \%$ increase in accuracy from the base model. Body mass $(b=-1.86, \mathrm{OR}=0.16, \mathrm{Wald}=7.00, \mathrm{p}<.0195 \% \mathrm{CI}[.04-$ $.62])$, gait speed $(b=0.42$, Wald $=6.35 \mathrm{OR}=1.52, \mathrm{p}<.0595 \% \mathrm{CI}[1.1-2.11])$, stride length $(b=-2.63, \mathrm{OR}=0.7, \mathrm{Wald}=9.19, \mathrm{p}<.0195 \% \mathrm{CI}[.01-0.395])$, and fall frequency $(b=-.286$, $\mathrm{OR}=.75, \mathrm{Wald}=4.14, \mathrm{p}<.05,95 \% \mathrm{CI}[.57-.99)$ were significantly different for decreased movement treated chicks when compared to normal movement treated chicks. The odds of a chick being successfully classified as receiving decreased movement treatment decreased as body mass and stride length increased. Further, as fall frequency increased the odds of being classified as receiving decreased movement treatment as opposed to normal movement treatment decreased. Whereas, chicks who locomoted faster were 1.5 times more likely to be successfully classified in the decreased movement treatment as opposed to normal movement treated chicks.

When considering the increased prenatal movement treatment classification as opposed to decreased movement prenatal treatment, body mass $(b=-2.82, O R=0.06$, Wald $=11.16, \mathrm{p}<.001,95 \% \mathrm{CI}[.01-.31])$, gait speed $(\mathrm{b}=.74, \mathrm{OR}=2.09$, Wald=11.97, $\mathrm{p}<.001,95 \% \mathrm{CI}[1.38-3.17])$, step length $(\mathrm{b}=-3.62, \mathrm{OR}=.03$, Wald $=10.28, \mathrm{p}<.001$, $95 \% \mathrm{CI}[.003-.25])$, and step width $(\mathrm{b}=-11.65$, OR $<0.001$, Wald $=11.85, \mathrm{p}<.001$, 
95\% CI[0 - .001]), significantly aided in accurate classification. Decreasing step width, step length, and body mass increased the odds of being classified into the increased movement treatment. Whereas, an increase in chicks speed showed 2 times increase in the odds of receiving an increased movement treatment classification as opposed to normal movement treatment classification.

\section{Discussion}

Here in we provide evidence for the importance of prenatal movement for motor development in 24-hour old bobwhite quail hatchlings. Our results indicate that quail embryos who experienced decreased or increased movement during early incubation showed a notable perturbation in postnatal body mass, and motor skill development 24hours after hatching. As anticipated, chicks who were exposed to decreased prenatal movement weighed less and showed minor delays in gait skill acquisition compared to chicks who received normal prenatal movement. Further, chicks who experienced decreased prenatal movement stimulation also showed delays in hatching. The delay in hatch results follow those reported for meat poultry (Boleli et al., 2016; Deeming, 1989; 2009; Tullett \& Deeming, 1987). Contrary to our predictions, chicks who were exposed to increased prenatal movement stimulation also showed decreased body weight and perturbations in motor performance; interestingly, these outcomes occurred despite hatching within the normal developmental window (E23). Moreover, body weight and gait outcomes were sensitive enough to accurately predict chick's prenatal movement treatment. Thus, our study identifies incubation movement as a maternal effect which contributes to postnatal motor outcomes at hatching. 
In chicken embryos, egg turning is closely associated with hatchability (Deeming, 2009; Robertson, 1961), with optimal rates of hatchability occurring at 96 turns per day throughout incubation (Robertson, 1961). Field reports corroborate that wild chickens turned their eggs roughly every 15 mins during the day (Olsen, 1930), resulting in approximately 96 turns per day. Robertson (1961) further reported increased turning rates, up to 480 turns per day, only showed slight decreases in hatchability relative to the optimal frequency; whereas, decreased rates, down to 0 turns per day, showed significantly large decreases in hatchability. Moreover, Deeming (1989) found that egg turning during the first week of incubation was critical for the development of the blood vessels near the eggshell, which facilitated gas exchange and nutrient uptake from the yolk. In our study, chicks received their assigned turning treatment for only seven days during the second week of incubation and normal turning experience (1 turn every two hours), during all non-treatment periods. During the seven-day treatment period, increase movement treated birds received 120 turns per day; whereas, decreased movement treated birds received 6 turns per day. Similar rates of hatchability between the increased and decreased treatments suggest the extreme turning regimens did not interfere with vascular development, which typically develops during the first week of incubation, but the variation in incubation length and the number of sickly birds reported in the decreased movement treatment suggests prenatal movement does generally influence development.

Several experiments have highlighted the importance of the albumen (egg white) for nutrition and growth (Everaert et al., 2013; Hill, 1993; Willems et al., 2014; Willems et al., 2015). In domestic fowl the albumen is regarded as the main source of water and represents $67 \%$ of the protein available for developing embryos (Willems et al, 2014). 
The fluid from the albumen flows into the yolk sac to form the subembryonic fluid (SEF) which surrounds the developing embryo and supports the transfer of nutrients (Babiker \& Baggott, 1992; Deeming, 2009). Egg turning plays an important role in nutrient transfer. The lack of turning slows the distribution of fluid, thereby changing the protein and ion concentration found in the SEF and ultimately delaying development (Baggott, Deeming, \& Latter, 2008; Deeming, 2009; Lilienthal, Drotleff, \& Ternes, 2015). During the second half of incubation, once the fluid has been completely transferred to the yolk, the dehydrated albumen is then absorbed and comingles with amniotic fluid where it is ingested by the embryo and assimilated into tissue (Willems et al., 2014). However, the rate of absorption and subseqent ingestion is also dependent upon the mechanical stress of egg turning and ultimately influences total body mass at hatch (Deeming, 2009; Lilienthal et al., 2015; Tullett \& Deeming, 1987). According to Deeming (2009) the cessation of turning prior to the albumen being absorbed into the amniotic fluid (on E12 in domestic chickens) has negative consequences to postnatal body mass, while the cessation of turning after absorption, but prior to ingestion (on E16 in domestic chickens), has little effect on postnatal body mass. In our study, the movement treatments began during the second week of incubation which corresponds to the onset of the second phase of incubation for quail (Yoshizaki, Ito, Hori, Saito, \& Iwasawa, 2002). As reported by Yoshizaki and colleagues (2002) the absorption process for quail begins on E8 but the digestion process is not complete until E16. Therefore, the decreased movement treatment, in the present study, likely interfered with the absorption process and contributed to the observed reduction in body mass. However, both treatment conditions showed a reduction in body mass. Very few studies have investigated the effects of 
increased egg turning on albumen absorption, making it less clear if the reduction in weight in the increased movement treated chicks was due to alter albumen absorption processes. Additional research is necessary to assess the effects of increased turning on the albumen absorption process.

The second week of incubation is also a sensitive period for the development of the vestibular reflex, which connects specialized hair cell mechanosensors of the innerear to brainstem interneurons which then transform and transmits the signals from hair sensors to motor neurons (Ginzberg \& Gilula, 1980; Represal, Van De Water, \& Bernd, 1991). The mechanosensors of the inner-ear provide information about angular and linear motion of the head, which facilitates postural control, balance, and head and eye movement; all necessary components of motor performance. In chickens, the hair cells of the vestibular system begin to develop around E3 (Ginzberg \& Gilula, 1980; Gottlieb, 1971) with chemical synapse with interneurons forming between E5 -E9 (Glover, 2003; Gottesman-Davis, 2011). Stimulation to the vestibular interneurons can evoked motoric responses as early as E10 (Hamburger \& Balaban, 1963; Sharp, 2015). However, compound action potentials directly from vestibular sensory hair cells are not detectable until after myelination which occurs around E14 (Peusner \& Giaume, 1997). While there is not direct corroborating evidence that the chicken timeline is accurate for quail embryos, there are several morphological and physiological studies which suggest quail ontogeny follows a similar, but slightly elongated temporal pattern, as the incubation period for chickens is slightly shorter (21 days) compared to the incubation period for bobwhite quail (23 days) (Ainsworth et al., 2010; Hamburger \& Hamilton, 1951; Hendrickx \& Hanzlik, 1965; Jones \& Jones, 1996). Since the manipulation period for the 
current study began on E8 and continued through E14, it is possible that the movement treatments may have affected these processes, especially since gait analysis showed a difference in early postnatal motor performance.

Studies investigating altered vestibular feedback consistently show perturbations in motor performance. For example, adult pigeons who's vestibular hair cells were pharmacologically removed showed severe postural and head instability, making them incapable of successfully completing a previously learned gait task (Dickman \& Lim, 2004). Motor decline also occurs in young animals deprived of vestibular experience (Geisler \& Gramsbergen, 1998; Kenyon et al., 1988). Both young rats and chicks who experienced severe reductions in vestibular experience showed difficulties with postural control. Further, chick and rat studies investigating the effects of altered vestibular experience through microgravity found delays in gravistatic synapse development, but not linear acceleration-sensitive synapses (Fermin et al., 1996; Lychakov et al., 1993; Ronca et al., 2000), suggesting vestibular sensory development is plastic and adaptable to the environmental context. Interestingly, microgravity studies with chicken and quail chicks do not report motor delays (Fermin et al., 1996; Lychakov et al., 1993), whereas investigations with rat pups only detect delays after accounting for other compensatory sensory (e.g., proprioceptive and cutaneous) systems (Ronca et al., 2000), indicating that changes in vestibular perception associated with environmental factors may only have subtle effects on motor performance. In the current study, only subtle motoric delays were found in both the increased movement and decreased movement treatments, indicating that prenatal movement treatment does not cause severe harm to sensorimotor function. Nevertheless, our results do highlight the importance of prenatal vestibular 
experience for typical motor development. Specifically, increases or decreases in prenatal movement stimulation produces similar delayed outcomes as those reported in postnatal locomotor restricted chicken hatchlings (Muir \& Chu, 2002). However, unlike postnatal motoric enrichment treatments (Adkins, Boychuk, Remple, \& Kleim, 2006; Muir \& Steeves, 1995), an increase in prenatal movement stimulation does not facilitate motor skill development.

The present study provides evidence that both increased and decreased prenatal movement during early incubation can affect growth and early postnatal motor performance. Quail chicks exposed to increased or to decreased early incubation movement had locomotor perturbations (i.e., decreased stride length and increased gait speed) and reduced postnatal body mass. However, increased and decreased prenatal movement stimulation did not influence fall frequency. Quail chicks exposed to decreased prenatal movement stimulation presented higher rates of delayed hatching. Further, gait analysis paired with the growth measure was sensitive enough to detect and distinguish between prenatal movement treatments. We conclude that both increased and decreased prenatal movement stimulation has negative consequences on growth and subtle alternations early postnatal (24-hour) motor development in bobwhite quail chicks. 
Table 1

Descriptive summary of movement treatments and data inclusion criteria

\begin{tabular}{|c|c|c|c|c|c|c|c|c|c|}
\hline $\begin{array}{l}\text { Movement } \\
\text { Treatment }\end{array}$ & Set & Candled & Early & Ontime & Late & Tested & $\begin{array}{c}\text { No } \\
\text { Emerge }\end{array}$ & $\begin{array}{l}\text { No } \\
\text { Move }\end{array}$ & Total N \\
\hline Control & 75 & 8 & $6^{a}$ & 48 & 0 & 48 & 14 & 8 & 28 \\
\hline Decreased & 100 & 15 & 0 & 51 & $20^{a}$ & 66 & 20 & 12 & 34 \\
\hline Increased & 75 & 9 & $10^{\mathrm{a}}$ & 48 & 0 & 48 & 8 & 8 & 32 \\
\hline Total & 250 & 32 & 16 & 147 & 20 & 162 & 42 & 28 & 94 \\
\hline
\end{tabular}

Note: 5 unhealthy chicks from the decreased treatment were not tested. Set=total eggs incubated, Candled=non-developing eggs, Early= chicks who hatched on E22, Ontime=chicks who hatched on E23, Late=chicks who hatched on E24, Tested=healthy chicks administered gait task, No Emerge $=$ chicks who failed to exit the emergence box, No Move=number of chicks who failed to move after exiting the emergences box, Total $N=$ number of chicks retained for data analysis. $a=$ significant $(p<.05)$ between group difference. 
Table 2

Gait Measures means (standard error) by movement treatment

\begin{tabular}{l|ccc}
\hline \multirow{1}{*}{ Gait Measure } & \multicolumn{3}{|c}{ Incubation Movement Treatment } \\
Normal & Decreased & Increased \\
\hline ITJ Angle $\left(^{\circ}\right)$ & $70(4.21)$ & $75(3.53)$ & $83(3.97)$ \\
Stride Length (cm) & $3.28(.11)$ & $2.76(.09)^{\mathrm{a}}$ & $2.63(.11)^{\mathrm{a}}$ \\
Base of Support & $1.37(.04)$ & $1.41(.03)$ & $1.42(.03)$ \\
$(\mathrm{cm})$ & & & \\
Step Width (cm) & $.66(.04)$ & $.73(.03)^{\mathrm{b}}$ & $.51(.04)^{\mathrm{a}, \mathrm{b}}$ \\
Gait Speed (cm/s) & $6.07(.67)$ & $8.84(.60)^{\mathrm{a}}$ & $9.41(.66)^{\mathrm{a}}$ \\
Cadence (step/s) & $2.93(.36)$ & $3.46(.3)$ & $2.55(.34)$ \\
Falls (frequency) & $3.57(.65)$ & $1.85(.55)$ & $2.41(.62)$ \\
Activity (s) & $78(8.36)$ & $75(5.73)$ & $85(6.15)$ \\
\hline \hline
\end{tabular}

Means reflect prenatal movement treatment after statistically accounting for body composition, velocity and developmental age. $a=$ Significant differences $(p<.015)$ between normal movement (NM) treatment. $b=$ significant differences $(p<.015)$ between decreased (DM) and increased (IM) treatment. 


\section{Body Composition by Movement Treatment}

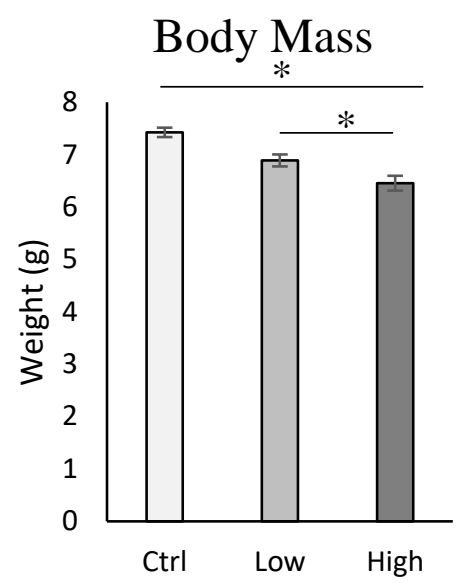

TARS Length

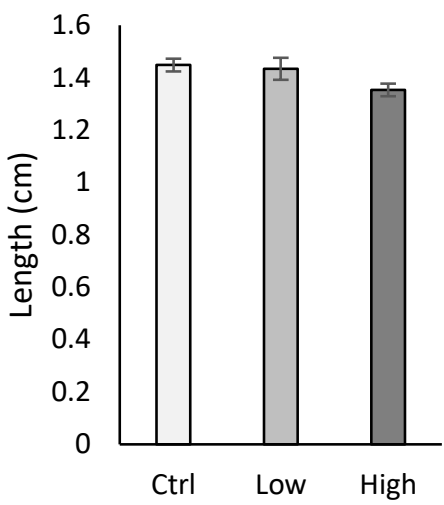

Figure 1

Body composition for movement treatments measured 24hours after hatching. Hatchlings were incubated under one of three prenatal movement conditions: control (ctrl) illustrated in white, decreased movement (Low) illustrated in light gray or, and increased movement (High) illustrated in dark gray.

Body mass measured in grams for each treatment.

Tarsometatarsus (TARS) length measured in centimeters for each. ${ }^{*} \mathrm{p}<.015$, vertical lines represent SE. 


\section{Probability of Movement Treatment}

A.

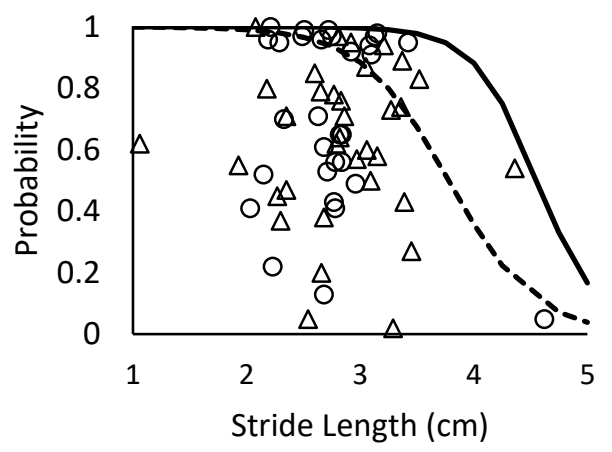

B.

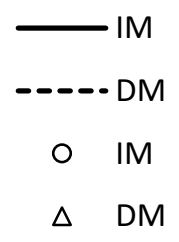

GAIT SPEED

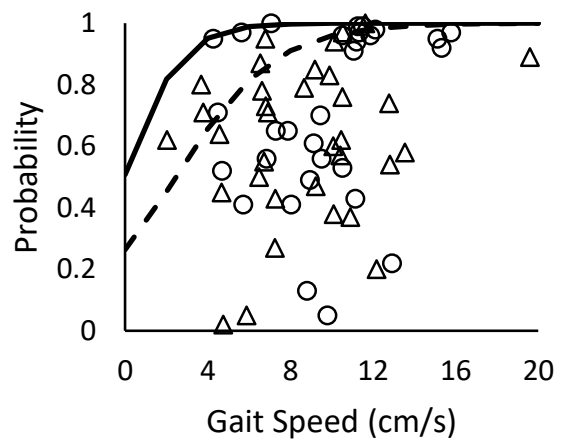

Figure 2

Predicted probabilities of movement treatment by stride length (A.) and gait speed (B.) measured 24-hours after hatching. During incubation embryos received either increased (IM), decreased (DM) or normal (NM) movement stimulation. The solid line represents the change in the predicted probability of IM classification (1) by stride length in comparison to the NM (0) classification. The circles represent actual probability outcomes of individual IM treated chicks. The dashed line represents the change in the predicted probability of DM classification by stride length in comparison to NM classification. The triangles represent actual probability outcomes of individual DM treated chicks. 


\title{
Study 3 \& Study 4
}

\section{PRENATAL LIGHT EXPOSURE INFLUENCES GAIT PERFORMANCE AND BODY COMPOSITION IN BOBWHITE QUAIL CHICKS}

This manuscript was published in the Journal of Physiology and Behavior on October 19, 2019

\author{
Starlie C. Belnap, M.S.*, Robert Lickliter, Ph.D. \\ Department of Psychology, Florida International University, Miami, FL 33199, USA
}

*Corresponding author: Starlie Belnap, sbelnap@fiu.edu, 305-348-1230, Department of Psychology, Florida International University, Miami, FL 33199.

\section{Acknowledgements}

This research was supported by National Science Foundation grant BCS 1525371 awarded to RL. We thank all the student researchers who assisted with this project, especially Amanda Del Risco, Taelor Aasen, and Abdullah Ahmad. 


\begin{abstract}
Maternal nesting behavior provides key elements essential for avian embryonic development. For example, avian research consistently shows the importance of prenatal light exposure for several developmental processes; however, previous research has primarily focused on artificial light regimens (i.e., $24 \mathrm{hr}$, $0 \mathrm{hr}$ light). Comparatively less is known about how exposure to naturally occurring light patterns during incubation influence motor performance, body composition, and incubation length. Herein, we conducted two experiments which investigated the effects of prenatal light exposure on incubation length, body composition (i.e., body mass, and bone length), and gait performance in 1-day-old bobwhite quail hatchlings. Study3 investigated discontinuous light exposure during the last two days of incubation under two light exposure treatments ( $2 \mathrm{hr}$ vs $6 \mathrm{hr}$ ) compared to a $12 \mathrm{hr}$ continuous light schedule. Results indicated discontinuous prenatal light experience extended the incubation period for $2 \mathrm{hr}$ exposed embryos, but not for 6hr exposed embryos, and negatively influenced postnatal body composition and postnatal gait performance when compared to $12 \mathrm{hr}$ exposed embryos. Study 4 examined the influence of prenatal light duration (2hr vs $6 \mathrm{hr}$ ) and light presentation (discontinuous vs sporadic). Results demonstrated sporadic light presentation improved gait performance in $2 \mathrm{hr}$ exposed hatchlings, but not $6 \mathrm{hr}$ exposed hatchlings, improved body composition in $6 \mathrm{hr}$ exposed hatchlings, but not $2 \mathrm{hr}$ exposed hatchlings, and did not alter incubation length when compared to discontinuous light counterparts. Our study provides further evidence for the importance of maternally regulated sensory stimulation during the prenatal period for early motor performance. Keywords: avian, prenatal light, kinematics, motor, body composition, incubation
\end{abstract}




\section{General Introduction}

Avian parents sit on their nests, getting up occasionally to reposition eggs, forage for food, or protect the nest from predation (Carroll et al., 2018; Kendeigh, 1952; Orcutt $\&$ Orcutt, 1976). The periodic episodes when avian parents are away from the nest are called inattentive periods (Kendeigh, 1952; Orcutt \& Orcutt, 1976). During early incubation, ground nesting precocial birds (i.e., ducks, chickens, quail) have periods of inattention during the early morning and late evening hours, while during late incubation inattentive periods shift to mid-day (Kendeigh, 1952). Moreover, inattentive periods become progressively increased over the course of incubation with long nest absences occurring late during incubation (Kendeigh, 1952; Orcutt \& Orcutt, 1976). Nesting behavior thus exposes developing precocial avian embryos to sporadic episodes of prenatal light experience, primarily during late incubation, contributing to species-typical phenotypic variability (Casey \& Sleigh, 2014; Rogers, 2014).

Research with chickens and quail consistently indicate the importance of prenatal light exposure for several developmental processes, including incubation duration (Lauber, 1975; Siegel, Isakson, Coleman, \& Huffman, 1969), metabolic function (Cooper, Voss, Ardia, Austin, \& Robinson, 2011; Gimeno, Roberts, \& Webb, 1967), body composition (Rozenboim, Piestun, et al., 2004; Sindhurakar \& Bradley, 2012), hemispheric lateralization (Ocklenburg \& Güntürkün, 2012; Rogers, Zucca, \& Vallortigara, 2004), and motor development (Belnap \& Lickliter, 2017; Bradley \& Jahng, 2003; Sindhurakar \& Bradley, 2012). Further, research indicates that within four days of incubation duck embryos can respond to light exposure, well before the development of the visual system (Gottlieb, 1968). Vallortigara and colleagues $(2013 ; 2019)$ found that 
early prenatal light exposure or lack of light exposure can affect gene expression in photosensitive regions, which affects the development of cerebral lateralization. Similarly, studies investigating light exposure during late-stages of incubation, following the development of the visual system, have demonstrated visual-motor asymmetries with as little as 2.5 to 6 hours of total light experience (Rogers, 1990). Prenatal light exposure has also been shown to accelerate development, thereby shortening the incubation period. Embryos who experience $24 \mathrm{hrs}$ of continuous light hatch one day earlier when compared to embryos who experience 12hrs of continuous light (Lauber, 1975; Siegel et al., 1969). In contrast, embryos who experience complete darkness hatch one day later when compared to embryos who experience 12hrs of continuous light (Lauber, 1975; Siegel et al., 1969).

Research has also indicated that the color, intensity, and timing of prenatal light experience can affect the incubation period (Rozenboim, Piestun, et al., 2004;

Sindhurakar \& Bradley, 2012) and can have long term effects on musculoskeletal physiology (Rozenboim, Biran, et al., 2004; Sindhurakar \& Bradley, 2012; van der Pol et al., 2019; L. Zhang et al., 2012). For example, adult fast-growing male broiler chickens incubated under monochromatic green LED (light emitting diode) light on an intermittent $15 \mathrm{~min}$ on/15min off schedule showed an increase in total body mass and breast muscle compared to broiler chickens incubated in the dark (Rozenboim, Piestun, et al., 2004). Further, common chicken embryos incubated under continuous full-spectrum white light showed longer tibia lengths compared to embryos incubated in the dark (Sindhurakar \& Bradley, 2012), while faster growing broiler chicken embryos incubated under 
continuous white LED light showed shorter tibia lengths compared to dark incubated embryos (van der Pol et al., 2019).

Since chickens and quail are precocial walkers and are able to maneuver and explore their surrounding within a few hours after hatching, they are a useful model for examining the effects of prenatal light exposure on early motor development. Several studies have shown that controlled stepping is established 1 to 3 days before hatching (Bradley, Solanki, \& Zhao, 2005; Ryu \& Bradley, 2009), with symmetric inter-limb alternations improving with light experience (Sindhurakar \& Bradley, 2012). Kinematic evaluations of newly hatched chickens who experienced $24 \mathrm{hrs}$ of full spectrum light showed that chicks performed similarly to those incubated under $12 \mathrm{hrs}$ of light or complete darkness (Sindhurakar \& Bradley, 2010), despite differences in developmental age. In other words, chicks who hatched a full day early performed similar to chicks who hatched a full day late (Sindhurakar \& Bradley, 2010). A recent study conducted in our laboratory using full spectrum white florescent light found similar locomotor competence in quail chicks incubated under $12 \mathrm{hrs}$ of continuous light or complete darkness, but a sharp decline in locomotor performance in quail incubated under $2 \mathrm{hrs}$ or $6 \mathrm{hrs}$ of discontinuous prenatal light during the last two days of incubation (Belnap \& Lickliter, 2017). Quail hatchlings in the discontinuous light conditions (2hrs or 6hrs) fell more frequently at hatch and 2-days later when compared to continuous light (12hrs or dark) conditions (Belnap \& Lickliter, 2017). However, their study did not address if developmental age, musculoskeletal physiology, or neurological control contributed to the observed differences. 
Gait and balance are complex sensorimotor behaviors which are sensitive to subtle changes in neuronal activity (Kannape \& Blanke, 2013; Pearson, 2004; Snijders et al., 2016). In humans, subtle changes in gait can predict the development of several diseases (Hausdorff, 2007; Pettersson, Olsson, \& Wahlund, 2005; Sawacha et al., 2012) and can help to clinically diagnose not only neuromotor disorders (Ebersbach et al., 1999; Pradhan et al., 2015), but also depression (Schrijvers, Hulstijn, \& Sabbe, 2008) and developmental disorders such as autism spectrum disorder (Cook, 2016; Travers, Powell, Klinger, \& Klinger, 2013). In animals, gait analysis is used to evaluate toxicology, neuroteratogens, and to elucidate mechanistic neural pathways (Kulig, 1996; Tecott \& Nestler, 2004; Tilson, 1993). Gait analysis appears to be a functional tool which can identify changes in neurological control, measure functional improvement, and inform therapeutic treatment.

The present study examined if gait performance, body composition, and developmental age in 1-day old bobwhite quail were influenced by the duration and pattern of presentation of prenatal light exposure. Specifically, in the first experiment we evaluated the role of discontinuous light duration using full spectrum white LED light under three light exposure conditions, $12 \mathrm{hrs}$ continuous (12C), $2 \mathrm{hrs}$ discontinuous (2D), and 6hrs discontinuous (6D). In the second experiment both the duration of the light exposure (i.e., $2 \mathrm{hr}$ and $6 \mathrm{hr}$ ) and the pattern of presentation of the light exposure (discontinuous vs. sporadic) were investigated. If gait performance and body composition are affected by prenatal light exposure, results would suggest that parental nesting behavior can influences prenatal motor development. 


\section{General Methods}

Fertilized bobwhite quail eggs (Colinus virginianus) were obtained weekly from a commercial game bird supplier (Strickland) and incubated in a GQF Sportsman Incubator with automatic turning under naturally low sunlight conditions (varied between 50-500 lux depending on weather and time of day) on approximately a $12 \mathrm{hr}$ light/dark cycle until embryonic day 17 (E17). The day fertile eggs were initially placed in the incubator was recorded as embryonic day zero out of 23 (E0). On E8 and E15, eggs were candled to identify infertility and non-developing embryos. On E17, experimental eggs were randomly selected and transferred to portable incubators (Brinsea Octagon 20 Advance) outfitted with an external automatic turning cradle. The incubators and cradle were placed within a specially designed light stimulus enclosure and administered their designated prenatal light exposure treatment. All incubators were maintained at $37.5^{\circ} \mathrm{C} \pm$ $0.2^{\circ} \mathrm{C}$, with a relative humidity of $60-65 \%$.

The light stimulation cabinet $(0.61 \mathrm{~m} \times 0.61 \mathrm{~m} \times 1.72 \mathrm{~m})$ contained four equal sized chambers $(0.61 \mathrm{~m} \times 0.61 \mathrm{~m} \times 0.43 \mathrm{~m})$, each with independent doors for selectable access. Each chamber independently controlled the intensity, duration and presentation of a customizable LED strip lighting $(4.5 \mathrm{~m})$ capable of producing up to 10,000 lux within a single chamber without affecting neighboring chambers. Intensity, duration, and presentation of light for each chamber was controlled through an automated lighting system produced by Samsung Smartthings. Light intensity was set at 1600 lux,

replicating the light stimulus utilized by Belnap and Lickliter (2017), with the modification from florescent light to LED light. 
On day E20, eggs were removed from the turning cradle in preparation for hatching. Following hatching, chicks' distal phalanges were marked for individual identification (Belnap et al., 2019; Belnap \& Lickliter, 2017). Because we wanted to account for developmental age, hatchlings were transferred by light treatment group and by day of hatch (E22, E23, and E24) into clear plastic rearing tubs in clutches of 12-15 to simulate naturally occurring brooding conditions. Chicks who hatched on day 22 and did not receive the entire light exposure treatment were excluded from testing. Tubs were maintained in a sound-attenuated rearing room at $30^{\circ} \mathrm{C}$ on a $12 \mathrm{hr}$ light/dark cycle. Food and water were available ad libitum.

\section{Gait analysis task}

Hatchling' motor coordination was evaluated using a 5min gait task administered one day after hatch. The $5 \mathrm{~min}$ gait task consisted of a $1 \mathrm{~min}$ acclimation period within a dark emergence box $(10 \mathrm{~cm} \times 10 \mathrm{~cm} \times 10 \mathrm{~cm})$, followed by a $4 \mathrm{~min}$ free roam exploration period within a Plexiglass runway. One hour prior to testing, square reflective tape $(.5 \mathrm{x}$ $.5 \mathrm{~cm}$ ) was placed in the center of the right and left tarsal pads to enhance visibility of foot placement during video scoring. To decrease handling effects and stress, chicks were transported in an opaque plastic container to the testing runway. Only chicks who emerged from the box were evaluated for motor coordination. Following testing chicks were immediately returned to the rearing room, where they were weighed and the right tarsometatarsus (TARS) length was measured. The TARS length was measured as the distance between the notch at the ITJ and the juncture of the TARS and the first digit.

The entire 5min testing session occurred within a Plexiglas rectangle runway outfitted with a dark removable lid $(95 \mathrm{~cm}$ long x $13 \mathrm{~cm}$ wide x $20 \mathrm{~cm}$ tall) raised 
approximately one meter above the floor in the same manner as reported by Belnap and colleagues (2019). The Plexiglas rectangle runway sat within a wooden support frame housed in a temperature-controlled room $\left(30^{\circ} \mathrm{C} \pm 1^{\circ} \mathrm{C}\right)$. A speaker calibrated at $65 \mathrm{~dB}$ located opposite from the emergence box continuously played a bobwhite quail maternal call to motivate hatchlings to cross the runway during the test period. Cameras were positioned to capture a lateral and inferior view and recorded the gait task using Kinovea, an open source kinematic software program (see Belnap et al., 2019 for more details).

\section{Gait parameters}

Steps and falls were acquired using Datavyu (Datavyu Team, 2014). Steps were defined as controlled forward movement, (walk, ground run, or aerial run) relative to orientation of the hatchling's head (Belnap \& Lickliter, 2017) and excluded lateral movement (i.e. side steps used in turning), weight transfer, and posterior movement. Step cadence was defined as the ratio of steps per seconds of active movement. Falls were defined as unstable movement, which resulted in the upper thorax or head contacting the runway floor. Hatchlings in a crouched position resting their upper thorax on the runway floor, were not scored as falling.

Spatiotemporal and body kinematics parameters were measured on separate frame by frame viewing passes until 20 usable measurements of each parameter were collected using Kinovea and followed the same techniques described by Belnap and colleagues (2019). Stride length, the distance between steps of the same limb, step width, the distance between contralateral limbs steps, and stance width, the distance between left and right limbs during double limb support, were scored using the inferior view, while velocity and ITJ angles were captured using the lateral camera view. Chicks who failed 
to emerge from the start box or who failed to produce more than 10 useable data points due to lack of movement were excluded from analysis (see Table 1).

Stride length and step width were acquired at touch down, when the tarsal pad makes initial contact with the surface. Stance width was acquired during moments of rest. Rest was defined as 180 ( 3 seconds) frames of non-movement, including forward movement, falls, jumps, and other forms of instability. ITJ was measured at initial touchdown of the gait cycle to capture maximum leg extension. Measurements occurred when the hatchling's locomotion was perpendicular to the camera, providing a clear profile view without video distortion of the right limb (for more details see Belnap et al., 2019). Spatiotemporal data per subject per parameter were averaged and the coefficient of variation $(\mathrm{CV}=\mathrm{SD} / \mathrm{mean} * 100)$ was calculated (Searls, 1964), providing the percentage of variability for ITJ, stride length, step width, and stance width, resulting in a total of eight measures (i.e. stride length, CV stride length, step width, CV step width, stance width, CV stance width, ITJ, and CV ITJ). Velocity was calculated only during bouts of forward locomotion using the following formula, $=\frac{x_{2}-x_{1}}{t_{2}-t_{1}}$, reflecting the change in position over the change in time averaged across all locomotion bouts.

Four observers were assigned to each performance measure. Prior to scoring observers were trained until inter- and intra-rater reliability of $>85 \%$ was achieved. Upon completion of scoring, approximately $10 \%$ of videos were randomly selected to be rescored to verify accuracy for each measure. Observers were blind to light treatment and relied upon individual bird identification markings, which were void of treatment assignment information. 


\section{Study 3: The effect of discontinuous prenatal light exposure on body composition and gait performance}

Discontinuous light exposure has been shown to negatively influence motor coordination in quail chicks by increasing fall rate during the first few days after hatching (Belnap \& Lickliter, 2017). Since no differences were found between the $12 \mathrm{hrs}$ light exposure condition and the dark exposure condition in the original study (Belnap \& Lickliter, 2017), and complete darkness is ecologically implausible for quail, we removed the dark condition from the current study. The remaining three light exposure treatments, 12C, 2D, and 6D were tested using the gait task. The purpose of Experiment 1 was to replicate previous behavioral results (i.e., fall frequency and step cadence) and to determine if exposure to discontinuous light influenced developmental age, body composition, and motor performance. We expected 6D and 2D chicks would hatch one day late (E24). However, based on pervious findings with chickens, we did not anticipate developmental age would directly affect gait. Rather, we expected that developmental age would predict body composition, specifically that chicks who hatched one day later would have larger body mass and shorter tarsometatarsus (TARS) lengths. We anticipated behavior measurements would mirror results from our pervious study, such that $6 \mathrm{D}$ and $2 \mathrm{D}$ hatchlings would fall more frequently compared to hatchlings in the $12 \mathrm{C}$ treatment. Lastly, we predicted the 6D and 2D treatments would show difference in their gait patterns, which may help explain differences in motor trajectories observed by Belnap and Lickliter (2017). 


\section{Methods}

On E17 75 eggs were randomly selected and distributed into one of three portable incubators located within the light stimulus cabinet. Each incubator was assigned to a single light exposure treatment. This process was repeated every two weeks until 50 embryos were assigned to each treatment condition, with eggs originating from at least two different egg batches, utilizing a total of 150 eggs. For the $12 \mathrm{C}$ treatment, the light stimulus continued as before, following a $12 \mathrm{hr}$ on/off cycle, but within the portable incubator within the stimulus cabinet. For the discontinuous treatments, the eggs remained in darkness until E21, following the same regime described in Belnap \& Lickliter (2017). The 2D treatment received 30min of continuous light experience in the morning and 30min of continuous light experience in the evening for two days prior to hatch, equaling a total of $2 \mathrm{hr}$ of overall light exposure. Similarly, the 6D treatment received 90min of continuous light experience twice a day for two days, equaling $6 \mathrm{hr}$ of total light exposure. On hatch day (E23) embryos received no light experience.

Following hatching, neonatal quail motor coordination was evaluated using the $5 \mathrm{~min}$ gait analysis task.

Data Analysis. Because of some of the data did not have normal distributions, all behavioral (step cadence and fall frequency) and spatiotemporal parameters were bootstrapped with replacement for 2,000 iterations. Right and left limb measurements for stride length were evaluated using paired sample t-tests. Chi-square likelihood tests and simple binomial outcome tests were used to characterize the influence of prenatal light experience on developmental age and gait task emergence. Differences in body composition and velocity between light conditions were evaluated using one-way 
analysis of variance (ANOVA) and were controlled for in subsequent analysis.

Spatiotemporal parameters and behavior were analyzed using multivariate analyses of variance (MANOVA; three light conditions on eight gait measures and two behavioral measures) with developmental age, body composition and velocity listed as covariates. MANOVA analysis accounts for potential correlations between dependent variables allowing for differences to be assessed without increasing type I error (Warne, 2014). Significant gait variables were further analyzed using univariate Bonferroni post hoc analyses with alpha adjustment set at p-values 0.015 . Since the frequency of falls may be attributed to differences in activity between temperature treatment groups, the relationship between step cadence and fall frequency was evaluated using Pearson correlation. If the preceding relationship was significant then step cadence was included as an additional covariate in post hoc analyses for falls. All statistical tests were conducted using SPSS v.22. Statistical significance was set at alpha $.05 .95 \%$ confidence intervals and effect sizes were calculated and interpreted using guidelines suggested by Durlak (2009).

\section{Results}

\section{Hatch description}

Developmental age did differ between light treatment conditions $(\chi 2(4)=36.441$, $\left.\mathrm{p}<0.001, \varphi_{\mathrm{c}}=0.525\right)$, with $71 \%(\mathrm{n}=22)$ of the late hatches occurring in the $2 \mathrm{D}$ treatment and $29 \%(n=9)$ occurring in the 6D treatment, and all the early hatches occurring in the $6 \mathrm{D}$ treatment $(\mathrm{n}=2$; see Table 1$)$. Early hatches were excluded from analysis. Despite, the variability in light exposure, the majority of birds $(n=99)$ hatched on E23 $(\chi 2(2)=6.18$, $\mathrm{p}<0.05)$. When evaluating the likelihood of emerging during the gait task by light 
treatment, chi-square test results revealed chicks behaved similarly across all light exposure treatments $\left(\chi^{2}(4)=4.124, \mathrm{p}=0.127, \varphi_{\mathrm{c}}=0.177\right)$. The failure to move after exiting the start box was also similar across light treatments $\left(\chi^{2}(2)=0.69, \mathrm{p}=0.71, \varphi_{\mathrm{c}}=0.07\right)$.

\section{Developmental age \& body composition}

Evaluations of body mass found significant differences between light exposure conditions $\left(F(2,81)=28.03, p<0.001, \eta^{2}=0.41\right)$ and developmental age $(F(1,81)=1.65$, $\left.\mathrm{p}<0.001, \eta^{2}=0.02\right)$. Chicks is the $12 \mathrm{C}$ treatment $(\mathrm{M}=7.47, \mathrm{SE}=0.10, \mathrm{p}<.05,95 \% \mathrm{CI}[7.28$ - 7.66]) weighed significantly more than chicks in the $6 \mathrm{D}(\mathrm{M}=6.14, \mathrm{SE}=0.14, \mathrm{p}<.05$, $95 \% \mathrm{CI}[5.86-6.41])$ or $2 \mathrm{D}(\mathrm{M}=6.43, \mathrm{SE}=0.12, \mathrm{p}<.05,95 \% \mathrm{CI}[6.18-6.66])$ treatments (see Figure 1). Chicks who hatched on E23 (M=6.73, SE= 0.09, p<.05, 95\%CI[6.55 6.91]) weighed significantly more than chicks who hatched on $\mathrm{E} 24(\mathrm{M}=6.55, \mathrm{SE}=0.23$, $\mathrm{p}<.05,95 \% \mathrm{CI}[6.12-7.01])$. Also, significant differences were found for TARS length by light treatment $\left(\mathrm{F}(2,81)=14.77, \mathrm{p}<0.001, \eta^{2}=0.15\right)$ and developmental age $\left(\mathrm{F}(1,82)=4.54, \mathrm{p}<0.05, \eta^{2}=0.05\right)$. Chicks in the $12 \mathrm{C}$ treatment $(\mathrm{M}=1.41, \mathrm{SE}=0.05$, 95\% CI $[1.311-1.51])$ had longer TARS lengths compared to chicks in the 6D $(\mathrm{M}=1.22$, $\mathrm{SE}=0.03, \mathrm{p}<.015,95 \% \mathrm{CI}[1.14-1.25])$ and $2 \mathrm{D}(\mathrm{M}=1.31, \mathrm{SE}=0.03, \mathrm{p}<.015,95 \% \mathrm{CI}[1.26$ $-1.36]$ ) treatments (see Figure 1). Additionally, chicks in the 6D treatment had significantly shorter TARS lengths compared to chicks in the $2 \mathrm{D}$ treatment $(\Delta \mathrm{M}=-0.1$, $\mathrm{SE}=0.03, \mathrm{p}<0.015,95 \% \mathrm{CI}[-0.18--0.04])$. Chicks who hatched one day late $(\mathrm{M}=1.41$, $\mathrm{SE}=0.05, \mathrm{p}<.05,95 \% \mathrm{CI}[1.311-1.51])$ had longer TARS lengths compared to chicks who hatched on time $(\mathrm{M}=1.32, \mathrm{SE}=0.02, \mathrm{p}<.05,95 \% \mathrm{CI}[1.28-1.36])$. 


\section{Gait \& behavior}

Evaluations of velocity found no significant differences between light treatments $(\mathrm{F}(2,80)=1.47, \mathrm{p}=0.24)$ or developmental age $(\mathrm{F}(1,80)=0.16, \mathrm{p}=0.69)$. Chick's average speed was $5.34 \mathrm{~cm} / \mathrm{s}(\mathrm{SE}=0.27)$ with a minimum speed of $1.27 \mathrm{~cm} / \mathrm{s}$ and a maximum speed of $14.21 \mathrm{~cm} / \mathrm{s}$ (see Table 2). Pearson correlation test between step cadence and falls revealed a non-significant negative relationship $(\mathrm{r}=-0.13, \mathrm{p}=0.23,95 \% \mathrm{CI}[-0.31-$ $0.11]$ ), indicating fall rate was not related to the number of steps taken when active. Because velocity is tightly related to step cadence and may influence fall frequency, velocity continued to be listed as a covariate in subsequent analysis. Furthermore, since there were no significant differences between the right $(\mathrm{M}=3.05, \mathrm{SD}=0.51)$ and left $(\mathrm{M}=3.06, \mathrm{SD}=0.50)$ limb stride length $(\mathrm{t}(89)=-0.265, \mathrm{p}=0.79)$, further analysis included only measurements for the right limb.

We ran a MANOVA with light treatments as the independent variable, 8 gait parameters and 2 behavioral measurements as the dependent variables, and velocity and developmental age as covariates. Of the covariates included in the model, only velocity significantly contributed to the overall model (Pillai's Trace $=0.238, \mathrm{~F}(10,68)=2.12$, $\left.\mathrm{p}<.05, \eta^{2}=0.24\right)$. However, body mass and TARS length contributed to individual parameters within the model. Velocity was significantly related stride length $(\mathrm{F}(1$, $\left.77)=7.96, p<.01, \eta^{2}=0.09\right)$, and stance width $\left(F(1,77)=6.38, p<.05, \eta^{2}=0.08\right)$, showing that faster hatchlings took longer strides and had significantly larger stance widths when at rest. Body mass significantly influenced $\mathrm{CV}$ stride length $\left(F(1,77)=6.99, \mathrm{p}<.01, \eta^{2}=0.08\right)$ with heavier hatchlings showing less variability in stride length. TARS length significantly influenced stride length $\left(F(1,77)=7.96, \mathrm{p}<.01, \eta^{2}=0.09\right)$ and fall frequency 
$\left(\mathrm{F}(1,77)=5.96, \mathrm{p}<.05, \eta^{2}=0.07\right)$. Chicks with shorter TARS lengths took longer strides and fell more frequently.

Light treatment continued to significantly contributed to the model (Pillai's Trace $\left.=0.45, \mathrm{~F}(20,138)=2.01, \mathrm{p}<.05, \eta^{2}=0.23\right)$ after accounting for the body composition and velocity. As shown in Table 2, stride length (Pillai's Trace $=0.24, F(2,77)=3.59, \mathrm{p}<.05$, $\eta^{2}=0.09$ ), stance width (Pillai's Trace $=0.26, \mathrm{~F}(2,77)=4.27, \mathrm{p}<.05, \eta^{2}=0.10$ ) and fall frequency (Pillai's Trace $=0.016, F(2,77)=3.4, \mathrm{p}<.05, \eta^{2}=0.08$ ) all significantly differed by light treatment, and step cadence (see Figure 2) trended toward significance (Pillai's Trace $\left.=0.05, \mathrm{~F}(2,77)=2.99, \mathrm{p}=.056, \eta^{2}=0.07\right)$. Chicks in the $6 \mathrm{D}(\mathrm{M}=3.12, \mathrm{SE}=0.07$, $\mathrm{p}<.05,95 \% \mathrm{CI}[-0.59--0.14])$ and $2 \mathrm{D}(\mathrm{M}=3.21, \mathrm{SE}=0.08, \mathrm{p}<.05,95 \% \mathrm{CI}[-0.6--0.13)$ treatments took significantly longer strides compared to the $12 \mathrm{C}$ treatment $(\mathrm{M}=2.76$, $\mathrm{SE}=0.09)$. Chicks in the 2D $(\mathrm{M}=1.46, \mathrm{SE}=0.04, \mathrm{p}<.05,95 \% \mathrm{CI}[1.38-1.54)$ treatment had significantly wider stance widths compared to chicks in the $12 \mathrm{C}$ treatment $(\mathrm{M}=1.24$, $\mathrm{SE}=0.06,95 \% \mathrm{CI}[1.12-1.37])$, but not compared to chicks in the $6 \mathrm{D}$ treatment $(\mathrm{M}=1.38$, $\mathrm{SE}=0.06,95 \% \mathrm{CI}[1.25-1.5)$.

When considering behavior, chicks in the $6 \mathrm{D}$ treatment $(\mathrm{M}=7.8, \mathrm{SE}=1.72, \mathrm{p}<.05$, $95 \% \mathrm{CI}[4.37-11.29)$ and 2D treatment $(\mathrm{M}=8.4, \mathrm{SE}=1.11, \mathrm{p}<.05,95 \% \mathrm{CI}[6.18-10.62)$ fell significantly more compared to the $12 \mathrm{C}$ treatment $(\mathrm{M}=2.21, \mathrm{SE}=1.21,95 \% \mathrm{CI}[0.03-$ 5.45), which replicated the findings of Belnap and Lickliter (2017) (see Figure 2). Lastly, posthoc evaluations of step cadence indicated that chicks in the $2 \mathrm{D}(\mathrm{M}=2.77, \mathrm{SE}=0.16$, $95 \% \mathrm{CI}[2.44-3.10)$ took significantly more steps per second compared to the $6 \mathrm{D}$ light treatment $(\mathrm{M}=2.19, \mathrm{SE}=0.24,95 \% \mathrm{CI}[1.67-2.69])$, but not when compared to the $12 \mathrm{C}$ 
treatment $(\mathrm{M}=2.32, \mathrm{SE}=0.24,95 \% \mathrm{CI}[1.84-2.8)$, indicating that chicks in the $2 \mathrm{D}$ treatment were more active than their $6 \mathrm{D}$ and $12 \mathrm{C}$ counterparts.

\section{Discussion}

The findings from Experiment 1 replicated previous findings and extended them by identifying that discontinuous light duration did influence developmental age, body composition and gait. As anticipated, hatchlings who experienced $2 \mathrm{hrs}$ of discontinuous light were more likely to hatch late, but surprisingly hatchlings in the 6D treatment had relatively few late hatches, which suggests that $6 \mathrm{hrs}$ of total light presented in $90 \mathrm{~min}$ bouts during the last two days of incubation was sufficient to accelerate development and stimulate the hatching process. Studies have consistently demonstrated that light stimulation increases oxygen consumption and heart rate (Gimeno et al., 1967; Lauber, 1975), whereas more recent research has identified that light exposure during incubation affects neuroendocrine signals through the cyclic production of neurohormones such as

melatonin, corticosterone, and thyroid hormones (Khalil, 2009; Özkan et al., 2012; Tong et al., 2018). Interestingly, these hormones also play a significant role in physiological metabolic processes and embryonic growth performance (Khalil, 2009; Özkan et al., 2012; Tong et al., 2018; Yu et al., 2018).

In the current study, light exposure did influence body composition such that chicks who experienced discontinuous light had smaller body mass and shorter TARS lengths, partially following the results reported by Sindhurakar and Bradley (2012). Moreover, chicks with smaller body mass showed more stride length variability and chicks with shorter TARS lengths took longer strides and fell more frequently, suggesting body composition is an important factor in locomotion performance. Overall, this study 
confirmed discontinuous $2 \mathrm{hr}$ and $6 \mathrm{hr}$ white light exposure during late incubation negatively influenced postnatal motor performance and body composition.

\section{Study 4: The effect of prenatal light exposure duration and presentation pattern on body composition and gait performance}

Within their natural environment, light exposure for developing quail embryos occurs in sporadic episodes during periods of maternal inattention. These bouts of inattention, while longer during late incubation, typically do not exceed 20mins (Mills, Crawford, Domjan, \& Faure, 1997). In Experiment 1, embryos were exposed to 30 or 90 minutes of light only twice a day, which likely contributed to the observed decline in motor performance. The purpose of Experiment 2 was to identify if poor motor performance observed in Experiment 1 resulted from the duration of the light exposure (i.e. $2 \mathrm{hr}$ or $6 \mathrm{hr}$ ), the presentation pattern of the light exposure (i.e. discontinuous or sporadic), or the combination of the both ( $2 \mathrm{hr}$ sporadic, $2 \mathrm{hr}$ discontinuous, $6 \mathrm{hr}$ sporadic, or $6 \mathrm{hr}$ discontinuous). Using a $2 \times 2$ (duration $\mathrm{x}$ presentation) factor model we evaluated developmental age, body composition, gait performance, and motor behavior in 1-day old bobwhite quail hatchlings. Given that sporadic light experience is what embryos typically experience in the wild, we anticipated motor performance would improve in the sporadic light exposure treatments, with a reduction in falls and gait variability compared to the discontinuous light pattern. However, we were unsure how the sporadic presentation light would affect body composition and developmental age, since the majority of research investigating body composition in birds has focused on meat production, rather than fitness. Because hatchlings in the $2 \mathrm{hr}$ condition appeared to better stability at 48hrs in Belnap and Lickliter (2017) and wider stance widths from 
Experiment 1, we further anticipated an interaction effect such that chicks in the $2 \mathrm{hr}$ sporadic treatment would show the greatest gains in motor performance.

\section{Methods}

Experiment 2 utilized the discontinuous data collected from Experiment 1 and added two additional prenatal light conditions, $2 \mathrm{hr}$ sporadic (2S) and the $6 \mathrm{hr}$ sporadic (6S). 50 eggs were randomly selected and equally distributed into one of two portable incubators located within the light stimulus cabinet. This process was repeated until 50 embryos were assigned to each light exposure treatment with eggs originating from at least two different egg batches, utilizing a total of 100 eggs. Embryos in the sporadic light treatments received several short bursts of light experience across a $12 \mathrm{hr}$ period, mimicking natural hen nesting behavior. Each burst was between 5 to 15 minutes in duration and pseudo-randomly occurred throughout a 12-hour period (7am to $7 \mathrm{pm}$ ), with at least 15 minutes of darkness between each burst of light experience. Depending on the light duration treatment ( $2 \mathrm{hr}$ or $6 \mathrm{hr}$ ), embryos received either 60 -min or 180-min of light exposure per day for the last two days prior to hatch. Following hatching, neonatal quails' motor coordination was evaluated using the 5min gait analysis task as described in Experiment 1.

Data Analysis. Pearson Chi-square tests were used to evaluate significant differences in developmental age between for light duration and for presentation type, and to assess the likelihood to emerge from the start box during the gait task. Differences in body composition and velocity between prenatal light factors were evaluated using $2 \times 2$ factor analysis of covariance (ANCOVA) with developmental age listed as a covariate. All behavioral (step cadence and fall frequency) and spatiotemporal gait parameters were 
bootstrapped with replacement for 2,000 iterations. Right and left limb measurements for stride length were evaluated using paired sample t-tests. Subsequent analyses controlled for body composition, developmental age, and velocity by including them as covariates within the model. Spatiotemporal parameters and behavior were analyzed using $2 \times 2$ multivariate factor analyses of variance (MANOVA; prenatal duration, prenatal presentation type, and their interaction on eight gait measures and two behavior measures). Significant gait variables were further analyzed using univariate techniques for two-factor models including simple effects tests.

\section{Results}

\section{Hatch description}

Chi-square analysis with developmental age and light duration revealed a significant effect of light duration on developmental age $\left(\chi^{2}(2)=43.51, p<.001, \varphi_{c}=0.5\right)$ (see Table 3). Of the chicks who hatched late (E24), 84\% ( $\mathrm{n}=48$ ) received $2 \mathrm{hr}$ of prenatal light and $16 \%(\mathrm{n}=9)$ received $6 \mathrm{hrs}$ of prenatal light experience. In contrast, presentation type did not reveal significant differences $\left(\chi^{2}(2)=1.30, p=0.52, \varphi_{c}=0.09\right)$ for developmental age. Chicks in the sporadic treatments hatched late $46 \%(n=26)$ of the time, whereas chicks in the continuous type hatched late $54 \%(n=31)$ of the time. Early hatches were excluded from analysis. When considering the influence of prenatal light exposure on the likelihood to emerge in testing trials Pearson chi-square results revealed no significant differences between light duration $\left(\chi^{2}(1)=1.62, \mathrm{p}=0.20, \varphi_{\mathrm{c}}=.10\right)$ or light presentation type $\left(\chi^{2}(1)=0.56, p=0.46, \varphi_{c}=.06\right)$. Of the chicks that did emerge, 14 failed to move. These chicks were distributed across all the light treatment conditions, with no 
reported difference between light duration $\left(\chi^{2}(1)=0.002, p=0.97, \varphi_{c}=.002\right)$ or presentation type $\left(\chi^{2}(1)=0.36, p=0.55, \varphi_{c}=.05\right)$.

\section{Developmental age \& body composition}

Analysis of body mass found no significant differences with light duration $(\mathrm{F}(1,108)=0.28, \mathrm{p}=0.6)$, light presentation $(\mathrm{F}(1,108)=0.33, \mathrm{p}=0.7)$, or their interaction $(\mathrm{F}(1,108)=0.75, \mathrm{p}=.39)$. Chicks in the $2 \mathrm{hr}$ light treatments weighed $(\mathrm{M}=6.4$, S.E.= 0.11, $95 \% \mathrm{CI}[6.18-6.60])$ about the same as those in the $6 \mathrm{hr}$ light treatments $(\mathrm{M}=6.32$, S.E. $=$ 0.09. 95\% CI[6.14 - 6.49]) and chicks in the sporadic presentation treatment $(\mathrm{M}=6.4$, S.E. $=0.09,95 \% \mathrm{CI}[6.22-6.58])$ weighed about the same as those in the discontinuous presentation treatment $(\mathrm{M}=6.32$, S.E. $=0.1,95 \% \mathrm{CI}[6.14-6.50])$. Furthermore, weight did not vary by developmental age $(\mathrm{F}(1,108)=0.08), \mathrm{p}=0.78)$.

When considering TARS length, there was a significant effect of light duration $(\mathrm{F}(1,108)=5.08, \mathrm{p}<.05, \eta 2=.05)$, but not presentation type $(\mathrm{F}(1,108)=0.83, \mathrm{p}=0.36)$, or their interaction $(\mathrm{F}(1,108)=1.12, \mathrm{p}=0.29)$ as illustrated in Figure 3. Chicks in the $2 \mathrm{hr}$ light treatments $(\mathrm{M}=1.32, \mathrm{SE}=0.02,95 \% \mathrm{CI}[1.28-1.36])$ had longer TARS lengths than chicks in the $6 \mathrm{hr}$ light treatments $(\mathrm{M}=1.257, \mathrm{SE}=0.02,95 \% \mathrm{CI}[1.20-1.29])$, while chicks in the sporadic treatments $(\mathrm{M}=1.29, \mathrm{SE}=0.02,95 \% \mathrm{CI}[1.26-1.33])$ had similar TARS lengths as chicks in the discontinuous treatments $(\mathrm{M}=1.27, \mathrm{SE}=0.02,95 \% \mathrm{CI}[1.23$ $-1.31])$. Even though the omnibus interaction test was not significant, simple effects tests showed that $6 \mathrm{D}$ hatchlings $(\mathrm{M}=1.22, \mathrm{SE}=.03)$ had significantly shorter TARS lengths compared to 2D hatchlings $(\mathrm{M}=1.32, \mathrm{SE}=0.03 ., \Delta \mathrm{M}=-0.10, \mathrm{p}<0.015,95 \% \mathrm{CI}[-0.175$ - 0.018]). Furthermore, developmental age did significantly contributed to the model $\mathrm{F}(1$, $\left.108)=22.32, \mathrm{p}<.001, \eta^{2}=0.17\right)$; chicks who hatched late $(\mathrm{M}=1.41, \mathrm{SE}=0.03,95 \% \mathrm{CI}[1.32$ 
- 1.47]) had longer TARS lengths compared to chicks who hatched on time $(M=1.24$, $\mathrm{SE}=0.02,95 \% \mathrm{CI}[1.21-1.27])$.

\section{Gait \& behavior}

Evaluations of velocity found a significant effect for light presentation $(\mathrm{F}(1$, $106)=4.55), \mathrm{p}<.05, \eta 2=.04)$, but failed to detect a significant effect for light duration or the interaction. Chicks in the sporadic presentation $(\mathrm{M}=5.85 \mathrm{~cm} / \mathrm{s}, \mathrm{SE}=0.31$, $95 \% \mathrm{CI}[5.26-6.49])$ were faster compared to chicks in the discontinuous presentation $(\mathrm{M}=4.97, \mathrm{SE}=.29,95 \% \mathrm{CI}[4.4-5.52])($ see Table 4). The average chick speed was 5.54 $\mathrm{cm} / \mathrm{s}(\mathrm{SE}=.24)$ with a minimum of $1.27 \mathrm{~cm} / \mathrm{s}$ and a maximum of $14.21 \mathrm{~cm} / \mathrm{s}$. Speed did not vary due to developmental age $(\mathrm{F}(1,106)=.68), \mathrm{p}=.41, \eta 2=.006)$. A paired t-test comparison between the right and left limb stride length revealed no significant differences between limbs. Subsequent analysis included only the right limb values. Pearson correlation analysis revealed a very weak and nonsignificant negative relationship between falls and step cadence $(r=-0.01, \mathrm{p}=0 . .91,95 \% \mathrm{CI}[-0.19-0.19])$, indicating that chicks rate of falling was not related to the number of steps they took when active.

We ran a MANOVA with light duration and light presentation as independent variables, gait and behavior parameters as dependent variables and body composition, developmental age, and velocity as covariate. Of the covariates listed, body mass (Pillai's Trace $=0.231, \mathrm{~F}(10,94)=2.825, \mathrm{p}<.01, \eta 2=.23)$ and velocity (Pillai's Trace $=0.352, \mathrm{~F}(10$, $94)=5.10, \mathrm{p}<.01, \eta 2=.35)$ significantly contributed to the overall model. Body mass was significantly related to stride length $(F(1,103)=5.94, p<.05, \eta 2=0.06)$, stride length $C V$ $(F(1,103)=6.46, p<.05, \eta 2=0.06)$, stance width $(F(1,103)=5.67, p<.05, \eta 2=0.05)$ and 
fall frequency $(F(1,103)=4.18, p<.05, \eta 2=0.04)$, while velocity was related to nearly all parameters with the exception of ITJ angle, stride length $\mathrm{CV}$, step width $\mathrm{CV}$ and stance width CV. Despite not contributing to the overall model, developmental age and TARS length did contributed to select parameters. TARS length was significantly related to fall frequency $(F(1,103)=5.05, p<.05, \eta 2=0.05)$, and developmental age significantly influenced step width $(\mathrm{F}(1,103)=5.99, \mathrm{p}<.05, \eta 2=0.06)$.

After accounting for covariation, light duration (Pillai's Trace $=0.157, \mathrm{~F}(10$, 94) $=1.75, \mathrm{p}=.08, \eta 2=.16$ ) or presentation type (Pillai's Trace $=0.06, \mathrm{~F}(10,94)=0.63$, $\mathrm{p}=0.79, \eta 2=.06)$ did not significantly contribute to the overall model. However, light duration did individually influence select parameters. As illustrated in Figure 4, hatchings in the $2 \mathrm{hr}$ treatments $(\mathrm{M}=2.77, \mathrm{SE}=0.13,95 \% \mathrm{CI}[2.51-3.03])$ took on average significantly $\left(\mathrm{F}(1,103)=4.56, \mathrm{p}<0.05, \eta_{\mathrm{p}}{ }^{2}=0.04\right)$ more steps per second compared to hatchlings in the $6 \mathrm{hr}$ treatments $(\mathrm{M}=2.32, \mathrm{SE}=0.15,95 \% \mathrm{CI}[2.03-2.61])$. No other parameters significantly varied by light duration or presentation type (see Table 4 and Figure 4).

The interaction between light duration and presentation significantly contributed to the overall model (Pillai's Trace $=0.257, \mathrm{~F}(10,94)=2.825, \mathrm{p}<.01, \eta 2=.26$ ). Stride length $\left(\mathrm{F}(1,103)=8.88, \mathrm{p}<0.05, \eta_{\mathrm{p}}{ }^{2}=0.08\right)$, step width $\left(\mathrm{F}(1,103)=7.68, \mathrm{p}<0.05, \mathrm{\eta}_{\mathrm{p}}{ }^{2}=0.07\right)$, and falls $\left(\mathrm{F}(1,103)=3.94, \mathrm{p}<0.05, \mathrm{\eta}_{\mathrm{p}}{ }^{2}=0.04\right)$ showed simple effect differences, after accounting for body composition, developmental age, and velocity (see Table 4). Chicks in the $2 \mathrm{~S}$ light treatment $(\mathrm{M}=2.82, \mathrm{SE}=0.09,95 \% \mathrm{CI}[2.64-3.00])$ had significantly shorter strides compared to chicks in the $2 \mathrm{C}$ light treatment $(\mathrm{M}=3.14, \mathrm{SE}=0.08$., $\Delta \mathrm{M}=-$ $0.32, \mathrm{p}<0.05,95 \% \mathrm{CI}[-0.63--0.01])$, indicating better motor control. In contrast, chicks 
in the $6 \mathrm{~S}$ and $6 \mathrm{D}$ light treatments performed similarly (see table 4). For step width, chicks in the $6 \mathrm{~S}(\mathrm{M}=0.48, \mathrm{SE}=0.04,95 \% \mathrm{CI}[0.4-0.56])$ treatment had significantly narrower step widths in comparison to chicks in the $6 \mathrm{D}$ treatment $(\mathrm{M}=0.6, \mathrm{SE}=0.05 ., \Delta \mathrm{M}=0.12$, $\mathrm{p}<0.05,95 \% \mathrm{CI}[0.03-0.27])$, indicating a narrowing in their base of support, whereas chicks in the $2 \mathrm{hr}$ light treatment group did not differ. Lastly, for fall frequency, chicks in the $2 \mathrm{~S}$ treatment $(\mathrm{M}=4.12, \mathrm{SE}=1.21,95 \% \mathrm{CI}[1.72-6.53])$ showed a notable reduction in fall frequency when compared to $2 \mathrm{D}$ treatment chicks $(\mathrm{M}=8.38, \mathrm{SE}=1.04 ., \Delta \mathrm{M}=-4.26$, $\mathrm{p}<0.05,95 \% \mathrm{CI}[0.9-8.54])$.

\section{Discussion}

In this experiment we evaluated the role of prenatal light duration and pattern of presentation on the development of postnatal motor performance, body composition, and incubation period in one day old bobwhite quail hatchlings. Sporadic light presentation reduced the number of falls in the $2 \mathrm{hr}$ light exposure treatments as anticipated, but not in the $6 \mathrm{hr}$ light exposure treatments. When evaluating gait parameters, hatchlings in the $2 \mathrm{~S}$ condition showed a reduction in stride lengths, some improvement in step width (although not significant) while maintaining step cadence and velocity compared to 2D treatment chicks. Together these gait parameters indicate $2 \mathrm{~S}$ treatment chicks employed compensatory strategies to reduce the rate of falls when traversing the challenging runway surface. Contrary to our prediction, $6 \mathrm{~S}$ treatment chicks maintained their stride length, greatly narrowed their base of support, and showed a significant increase in velocity while maintaining a similar rate of stepping compared to $6 \mathrm{D}$ treatment chicks. Together these gait parameters indicate $6 \mathrm{~S}$ treatment chicks continued to show similar motor performance as 6D treatment chicks. 
When considering developmental age, the sporadic presentation of the light stimulus did not accelerate development. Hatchlings in the $2 \mathrm{hr}$ light exposure treatment continued to hatch one day later than those in the $6 \mathrm{hr}$ light exposure treatment. Further after for controlling for possible developmental age effects, chicks in the $2 \mathrm{hr}$ treatment had longer TARS lengths regardless of the light presentation type. However, the sporadic presentation of the light stimulus did influence hatchlings in the 6hr light exposure treatments; hatchlings' TARS length was longer in the $6 \mathrm{~S}$ treatment compared to $6 \mathrm{D}$ treatment hatchlings. Although not significant, a similar trend was also present for body mass in the $6 \mathrm{hr}$ light exposure chicks. Overall the results from this experiment confirm the importance of prenatal light duration and prenatal light presentation for body composition and motor performance in bobwhite quail. Specifically, the sporadic light presentation improved motor performance within the $2 \mathrm{hr}$ light treatments, and the sporadic presentation of light enhanced body composition within the $6 \mathrm{hr}$ light treatments.

\section{General Discussion}

The present study evaluated the effects of prenatal light experience on postnatal motor development, body composition, and incubation period. The results from Experiment 1 confirmed the deleterious effects of prenatal discontinuous light exposure on motor performance after accounting for the confounding influence of developmental age and differences in body composition in bobwhite quail hatchlings. Additionally, the results from Experiment 2 showed that the duration of light exposure and the pattern of presentation of light during the final days of incubation influenced postnatal body composition and motor performance during the gait task. These findings suggest that maternally regulated prenatal light exposure during incubation is an important factor for 
species-typical motor development. Specifically, the sporadic presentation of prenatal light experience, which mimicked naturally occurring hen nesting behavior, aided in optimizing body composition and motor performance within select light durations.

This study adds to the growing body of literature showing that maternally regulated prenatal sensory experience is important for species-typical development and phenotypic variability. This includes morphological development (Rozenboim, Biran, et al., 2004; Rozenboim, Piestun, et al., 2004; Serrat, 2014; Sindhurakar \& Bradley, 2012; C. M. Wilson \& McNabb, 1997), thermoregulatory ability (Collin et al., 2007; DuRant et al.,2013; Mariette \& Buchanan, 2016), locomotor performance (Belnap et al., 2019; Belnap \& Lickliter, 2017; Hopkins et al., 2011; Porterfield et al., 2015), behavioral reactivity (Archer \& Mench, 2017; Guenther \& Trillmich, 2013; Hope et al., 2018; Pittet et al., 2013; Reed \& Clark, 2011), and perceptual and cognitive development (Gottlieb et al., 1989; Lickliter, 2005; Lickliter et al., 2002; Roy et al., 2014). Each of these studies show fluctuations in sensory experience (i.e. temperature, light, or sound), which are typically regulated through parental care, can have significant effects on the developing avian embryo.

The motor system is one of the first functional systems to provide the growing embryo with both exogenous sensory information, which typically results from maternal stimulation, and endogenous sensory feedback, which typically results from selfgenerated movement. As a result, the motor system has the most extensive experiential history at hatching and lays the neurological foundation for subsequent sensory systems (Ainsworth et al., 2010; Gottlieb, 1971; Hamburger \& Balaban, 1963). Further, early embryonic spontaneous movements are known to be important for the formation of 
cartilage and bone (Hamburger, 1973; Muller, 2003; Nowlan et al., 2010), skeletal muscle development (Hall \& Herring, 1990; Nowlan et al., 2010), and facilitates synaptogenesis between interneurons and motorneurons within the spinal cord (Hamburger, 1973; Le Ray et al., 1993; Sharp, 2015). These early movements are highly susceptible to sensory stimulation. For example, a study conducted by Reynolds and Lickliter (2002) demonstrated E21 bobwhite quail embryos increased embryonic activity after unimodal auditory and visual stimulation and this effect was amplified when the visual and auditory systems were concurrently stimulated. A study conducted by Bradley and Jahng (2003) demonstrated motility increase in E18 chicken embryos who experienced continuous light compared to $12 \mathrm{hr}$ light/dark photoperiod during incubation. Hammond and colleagues (2007) further demonstrated chicken embryo activity was sensitive to small adjustments to ambient air temperature. Each of these sensory experiences are in part regulated through the maternal hen's nesting behavior.

In the current study, embryos' recurring episodic experience with the light stimulus over the course of a $12 \mathrm{hr}$ period (Experiment 2) may have elicited increased motility, while protecting the embryo from fatigue and habituation. Increased motility would not only affect motor performance, but also body composition. However, it is unclear if increased motility independent of light experience would affect the length of incubation. Previous research has confirmed a relationship between prenatal light experience and accelerated maturation of the motor system (Bradley \& Jahng, 2003; Sindhurakar \& Bradley, 2010, 2012) and other types of sensory stimulation (Hammond et al., 2007; Reynolds \& Lickliter, 2002), but to date no study has evaluated if increased motility alone is sufficient to reduce the incubation period. Further there is evidence that 
the relationship between light and motor activity persists throughout adulthood in chickens. Postnatal studies evaluating intermitted light schedules for meat chickens found that recurring $4 \mathrm{hr}$ light/dark cycle increased motoric activity, feed intake, tibia length, and suppressed myopathy (Lan, Feldkaemper, \& Schaeffel, 2014; Yang et al., 2015) compared to extended light, extended dark, or equal light/dark photoperiods.

In contrast, it is less clear how discontinuous light regimens (Experiment 1) may have affected prenatal motility. Unlike the current study, most research reports positive effects of prenatal light experiences on development (Akasaka, Nasu, Katayama, \& Murakami, 1995; Khalil, 2009; Lauber, 1975; Porterfield et al., 2015); however, few studies have investigated the influence of short photoperiods on embryogenesis. These studies typically compare $12 \mathrm{hrs}$ light/dark regimen to complete darkness. Most agree embryos incubated in complete darkness show reductions in activity and metabolic function which typically corresponds to arrested development and delayed hatching (Lauber, 1975; Siegel et al., 1969; Sindhurakar \& Bradley, 2012). However, this delay does not affect motor coordination at time of hatch (Sindhurakar \& Bradley, 2012). Instead, small motoric differences appear a few hours later while ambulating in the common chicken (Porterfield et al., 2015) and in the bobwhite quail (Belnap \& Lickliter, 2017). Also, postnatal studies investigating extended darkness periods (1hr light/23 hr darkness and 4hr light/ 26hr darkness) with limited light exposure reported similar declines in motor performance and body composition (see Olanrewaju et al., 2006 for a review). Broiler chicks reared under these conditions had smaller body mass, increased immobility and mortality rates (Olanrewaju et al., 2006). The reported decline was attributed to decreased activity and metabolic function induced by long periods of 
darkness. In the current study, 2D treatment chicks showed a significant delay in hatching, indicating arrested development, whereas the majority of 6D treatment chicks hatched on-time, but also showed motor and body composition decline. Other metabolic pathways influenced by light may be responsible for this observed decline and may explain the differential improvement observed in the sporadic light exposure treatments. Regardless of the mechanistic pathway, this study provides initial evidence of the importance of prenatal light duration and pattern of light presentation for incubation period, body composition, and motor performance in one day old bobwhite quail hatchlings. Findings from this study highlight the importance of species-typical nesting behavior on shaping offspring phenotype. Further investigation of these prenatal sensory experiences may help to identify which factors are necessary for optimal motor development. 
Table 1

Descriptive summary of incubation and data inclusion criteria for Experiment 1

\begin{tabular}{cc|ccc|ccc|c}
\hline $\begin{array}{c}\text { Light } \\
\text { Treatment }\end{array}$ & Set & Early & Ontime & Late & Tested & Emerge & No Move & Total N \\
\hline 12C & 50 & 0 & 45 & 0 & 45 & 12 & 5 & 28 \\
6D & 50 & 2 & 33 & 9 & 44 & 7 & 3 & 34 \\
2D & 50 & 0 & 21 & $22^{\text {a }}$ & 43 & 15 & 5 & 23 \\
Total & 150 & 2 & 99 & 31 & 132 & 34 & 13 & 85 \\
\hline \hline
\end{tabular}

$C=$ continuous light, $D=$ discontinuous light, Set=total number of eggs transferred to a light treatment,

Early=number of chicks who hatched on E22, Ontime=number of chicks who hatched on E23,

Late=number of chicks who hatched on E24, Tested=chicks who completed the gait task, No

Emerge $=$ number of chicks who failed to exit the emergence box, No Move=number of chicks who failed

to move after exiting the emergences box, Total $N=$ number of chicks retained for data analysis.

$a=$ significant $(p<.05)$ between group difference. 
Table 2

Experiment 1 means (standard error) for spatiotemporal parameters

\begin{tabular}{l|ccc}
\hline \multirow{1}{*}{ Gait Measure } & \multicolumn{4}{c}{ Incubation Light Treatment } \\
\hline ITJ Angle & $63.40(6.30)$ & 75.144 & $76.01(4.31)$ \\
& & $(6.66)$ & \\
ITJ CV & $19.52(2.26)$ & $24.38(2.39)$ & $22.35(1.55)$ \\
Stride Length & $2.76(.13)$ & $3.12(.14)^{\mathrm{a}}$ & $3.21(.09)^{\mathrm{a}}$ \\
Length CV & $24.56(1.77)$ & $21.68(1.86)$ & $22.94(1.21)$ \\
Step Width & $.62(.05)$ & $.59(.06)$ & $.53(.04)$ \\
Step CV & $33.87(3.08)$ & $37.52(3.26)$ & $33.26(2.12)$ \\
Stance Width & $1.24(.06)$ & $1.38(.06)$ & $1.46(.04)^{\mathrm{a}}$ \\
Stance CV & $22.19(1.53)$ & $24.27(1.62)$ & $22.34(1.05)$ \\
Velocity & $5.97(.49)$ & $4.60(.5)$ & $5.30(.42)$ \\
\hline ITJ angle=degrees, Stride Length=cm, Step Width=cm, Stance \\
Width=cm, CV=Coefficient of variation, a percentage of variability \\
for each kinematic parameter, Velocity= cm/s. Means reflect \\
prenatal light treatment after statistically accounting for body \\
composition, velocity and developmental age. a=Significant \\
differences (p<.015) between the 12hr continuous light treatment.
\end{tabular}


Table 3

Descriptive summary of incubation and data inclusion criteria for Experiment 2

\begin{tabular}{lc|ccc|ccc|c}
\hline Light Factor & Set & Early & Ontime & Late & Tested & Emerge & No Move & Total N \\
\hline $\begin{array}{l}\text { Duration } \\
\text { 6hr }\end{array}$ & 100 & 6 & $74^{\mathrm{a}}$ & $9^{\mathrm{a}}$ & 89 & 21 & 7 & 61 \\
2hr & 100 & 0 & 39 & 48 & 87 & 29 & 7 & 52 \\
$\begin{array}{l}\text { Presentation } \\
\text { D }\end{array}$ & 100 & 2 & 54 & 31 & 87 & 22 & 8 & 57 \\
S & 100 & 4 & 63 & 26 & 89 & 27 & 6 & 56 \\
Interaction & & & & & & & & \\
6D & 50 & 2 & 33 & 9 & 44 & 7 & 3 & 34 \\
2D & 50 & 0 & 21 & 22 & 43 & 15 & 5 & 23 \\
6S & 50 & 4 & 45 & 0 & 45 & 14 & 4 & 27 \\
2S & 50 & 0 & 18 & 26 & 44 & 13 & 2 & 29 \\
Total & 200 & 6 & 117 & 57 & 176 & 49 & 14 & 113 \\
\hline \hline
\end{tabular}

D=discontinuous light, $S=$ sporadic light, Set=total number of eggs transferred to the light conditions, Early=number of chicks who hatched on E22, Ontime=number of chicks who hatched on E23,

Late=number of chicks who hatched on E24, Tested=number of chicks actually tested, No

Emerge=number of chicks who failed to exit the emergence box, No Move=number of chicks who failed to move after exiting the emergences box, Total $N=$ number of chicks retained for data analysis.

$a=$ significant $(p<.05)$ between group difference. 
Table 4

Experiment 2 means (standard error) for spatiotemporal parameters

\begin{tabular}{|c|c|c|c|c|c|c|c|c|}
\hline \multirow[b]{2}{*}{ Gait Measure } & \multicolumn{2}{|c|}{ Light Duration } & \multicolumn{2}{|c|}{ Light Presentation } & \multicolumn{4}{|c|}{ Interaction } \\
\hline & $2 h r$ & $6 h r$ & $D$ & $S$ & $2 S$ & $2 D$ & $6 S$ & $6 D$ \\
\hline ITJ Angle & $\begin{array}{l}71.15 \\
(3.25)\end{array}$ & $\begin{array}{l}74.64 \\
(3.64)\end{array}$ & $\begin{array}{l}72.95 \\
(3.27)\end{array}$ & $\begin{array}{l}72.84 \\
(3.19)\end{array}$ & $\begin{array}{l}67.05 \\
(4.78)\end{array}$ & $\begin{array}{l}75.29 \\
(4.10)\end{array}$ & $\begin{array}{l}78.63 \\
(4.66)\end{array}$ & $\begin{array}{l}70.65 \\
(5.37)\end{array}$ \\
\hline ITJ CV & $\begin{array}{l}21.28 \\
(2.09)\end{array}$ & $\begin{array}{l}27.62 \\
(2.34)\end{array}$ & $\begin{array}{c}23.0 \\
(2.11)\end{array}$ & $\begin{array}{l}25.90 \\
(2.05)\end{array}$ & $\begin{array}{l}21.27 \\
(3.08)\end{array}$ & $\begin{array}{l}21.29 \\
(2.64)\end{array}$ & $\begin{array}{c}30.53 \\
(3.0)\end{array}$ & $\begin{array}{l}24.71 \\
(3.46)\end{array}$ \\
\hline Stride Length & $\begin{array}{l}2.98 \\
(.06)\end{array}$ & $\begin{array}{l}3.01 \\
(.07)\end{array}$ & $\begin{array}{l}3.03 \\
(.06)\end{array}$ & $\begin{array}{l}2.97 \\
(.06)\end{array}$ & $\begin{array}{l}2.82^{\mathrm{a}} \\
(.09)\end{array}$ & $\begin{array}{l}3.14^{\mathrm{a}} \\
(.08)\end{array}$ & $\begin{array}{l}3.11 \\
(.09)\end{array}$ & $\begin{array}{l}2.92 \\
(.10)\end{array}$ \\
\hline Length CV & $\begin{array}{l}24.82 \\
(1.01)\end{array}$ & $\begin{array}{l}24.20 \\
(1.13)\end{array}$ & $\begin{array}{l}23.91 \\
(1.02)\end{array}$ & $\begin{array}{c}25.12 \\
(.99)\end{array}$ & $\begin{array}{l}25.39 \\
(1.49)\end{array}$ & $\begin{array}{l}24.25 \\
(1.28)\end{array}$ & $\begin{array}{l}24.84 \\
(1.45)\end{array}$ & $\begin{array}{l}23.56 \\
(1.67)\end{array}$ \\
\hline Step Width & $\begin{array}{c}.56 \\
(.03)\end{array}$ & $\begin{array}{c}.54 \\
(.03)\end{array}$ & $\begin{array}{c}.56 \\
(.03)\end{array}$ & $\begin{array}{c}.54 \\
(.03)\end{array}$ & $\begin{array}{l}.60 \\
(.04)\end{array}$ & $\begin{array}{l}.51 \\
(.03)\end{array}$ & $\begin{array}{l}.48^{\mathrm{a}} \\
(.04)\end{array}$ & $\begin{array}{l}.60^{\mathrm{a}} \\
(.05)\end{array}$ \\
\hline Step CV & $\begin{array}{l}33.51 \\
(2.02)\end{array}$ & $\begin{array}{l}36.20 \\
(2.26)\end{array}$ & $\begin{array}{c}34.0 \\
(2.03)\end{array}$ & $\begin{array}{l}35.71 \\
(1.98)\end{array}$ & $\begin{array}{c}34 . \\
(2.97)\end{array}$ & $\begin{array}{l}33.02 \\
(2.55)\end{array}$ & $\begin{array}{c}37.43 \\
(2.9)\end{array}$ & $\begin{array}{l}34.98 \\
(3.34)\end{array}$ \\
\hline Stance Width & $\begin{array}{l}1.39 \\
(.03)\end{array}$ & $\begin{array}{l}1.37 \\
(.04)\end{array}$ & $\begin{array}{l}1.38 \\
(.03)\end{array}$ & $\begin{array}{l}1.37 \\
(.03)\end{array}$ & $\begin{array}{l}1.35 \\
(.05)\end{array}$ & $\begin{array}{l}1.43 \\
(.04)\end{array}$ & $\begin{array}{l}1.40 \\
(.05)\end{array}$ & $\begin{array}{l}1.33 \\
(.05)\end{array}$ \\
\hline Stance CV & $\begin{array}{c}22.96 \\
(.73)\end{array}$ & $\begin{array}{c}22.06 \\
(.82)\end{array}$ & $\begin{array}{c}22.05 \\
(.73)\end{array}$ & $\begin{array}{l}22.71 \\
(.72)\end{array}$ & $\begin{array}{l}23.32 \\
(1.07)\end{array}$ & $\begin{array}{c}22.06 \\
(.92)\end{array}$ & $\begin{array}{l}22.10 \\
(1.05)\end{array}$ & $\begin{array}{l}22.03 \\
(1.21)\end{array}$ \\
\hline Velocity & $\begin{array}{l}5.49 \\
(.27) \\
\end{array}$ & $\begin{array}{l}5.33 \\
(.32) \\
\end{array}$ & $\begin{array}{l}5.85^{\mathrm{a}} \\
(.31)\end{array}$ & $\begin{array}{c}4.97^{\mathrm{a}} \\
(.29) \\
\end{array}$ & $\begin{array}{l}5.64 \\
(.43) \\
\end{array}$ & $\begin{array}{l}5.33 \\
(.32) \\
\end{array}$ & $\begin{array}{l}6.07^{\mathrm{b}} \\
(.44)\end{array}$ & $\begin{array}{l}4.6^{\mathrm{b}} \\
(.48) \\
\end{array}$ \\
\hline
\end{tabular}

ITJ angle=degrees, Stride Length=cm, Step Width=cm, Stance Width=cm, CV=Coefficient of variation, a percentage of variability for each kinematic parameter, Velocity $=\mathrm{cm} / \mathrm{s}$. Means reflect prenatal light treatment after statistically accounting for body composition, velocity and developmental age. Each superscript the row indicates a significant pairwise comparison p<.015. 


\section{Body Composition: Experiment 1}

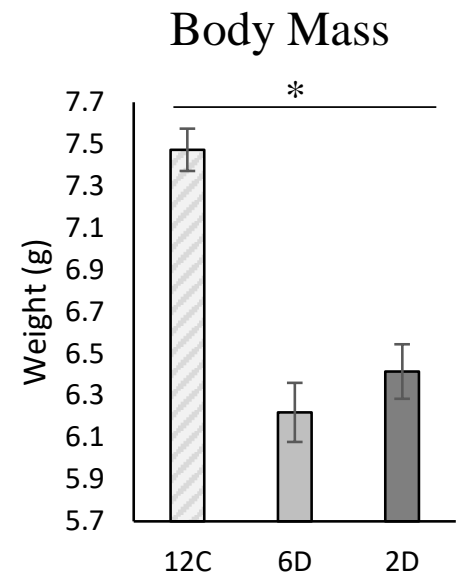

TARS Length

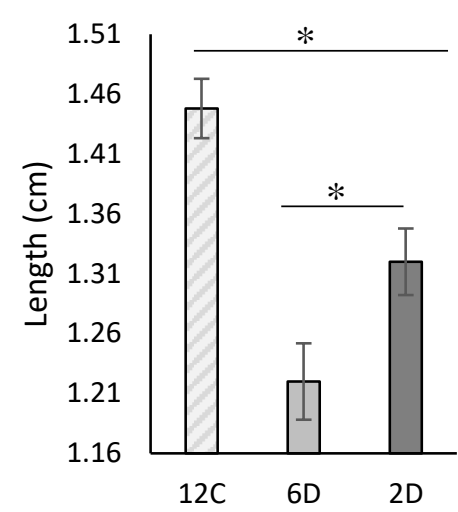

Figure 1

Body composition for Experiment 1 measured 24-hours after hatching. Hatchlings were incubated under one of three prenatal light conditions: $12 \mathrm{hr}$ continuous (12C) illustrated in strips, 6hr discontinuous (6D) illustrated in light gray or, $2 \mathrm{hr}$ discontinuous (2D) illustrated in dark gray. Body mass measured in grams for each light treatment. Tarsometatarsus (TARS) length measured in centimeters for each light treatment. ${ }^{*} \mathrm{p}<.015$, vertical lines represent SE. 


\section{Experiment 1: Behavior Measurements}

Step Cadence

Fall Frequency
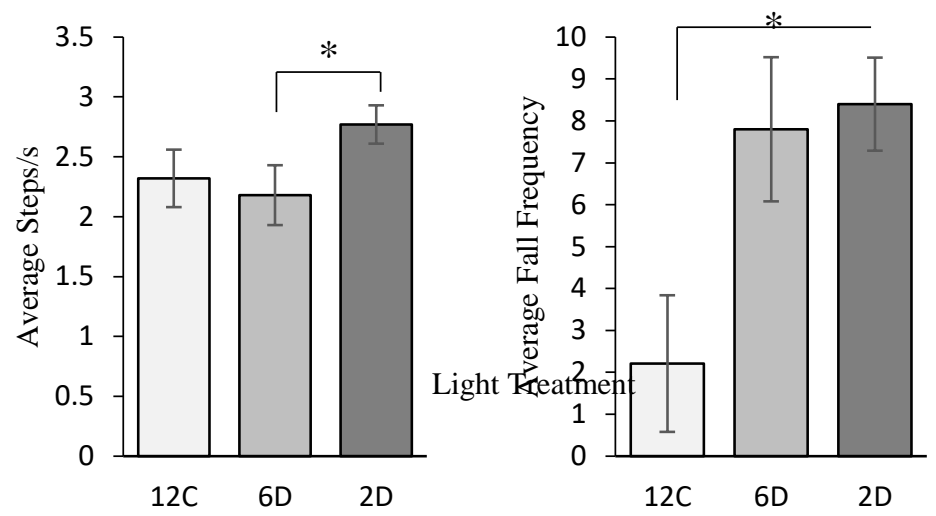

Figure 2

Behavioral measurements for each light treatment measured at 24-hours after hatching. All values account for differences in body composition, velocity and developmental age. The $12 \mathrm{hr}$ continuous (12C) prenatal light treatment is illustrated in white. The 6hr discontinuous (6D) prenatal light treatment is illustrated in light gray. The $2 \mathrm{hr}$ discontinuous (2D) prenatal light treatment is illustrated in dark gray. Step cadence reflects the average number of steps taken per second of activity. Falls frequency represents the number of falls per video. Vertical lines depict SE. $* \mathrm{p}<.015$. 
A.

\section{Body Composition: Experiment 2}

\section{Light Duration}

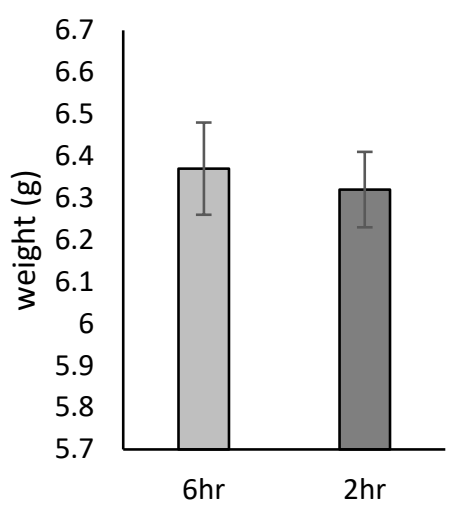

B. Light Duration

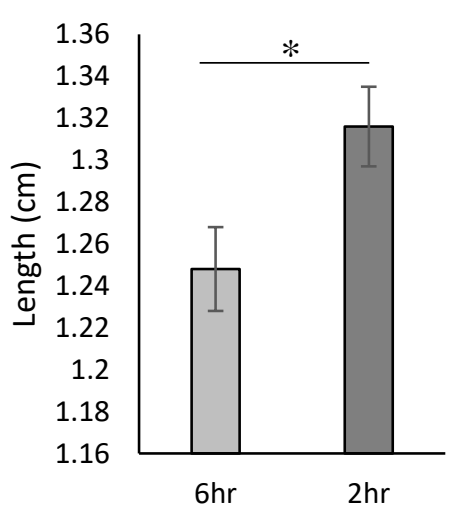

Light Presentation

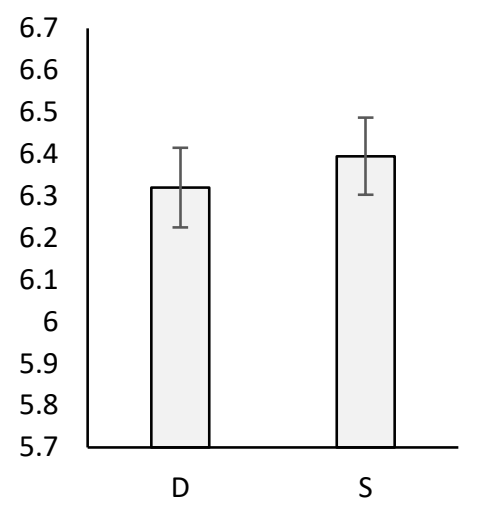

Light Presentation

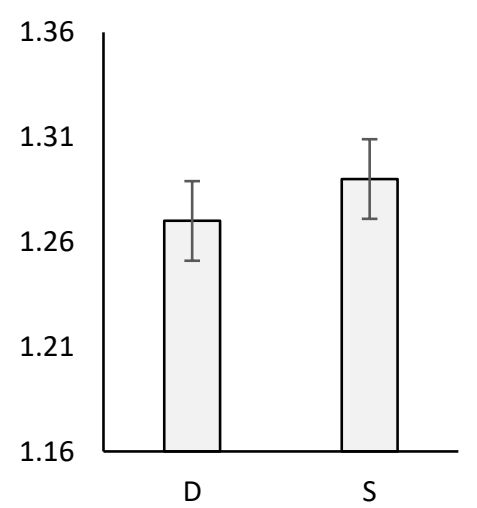

Interaction

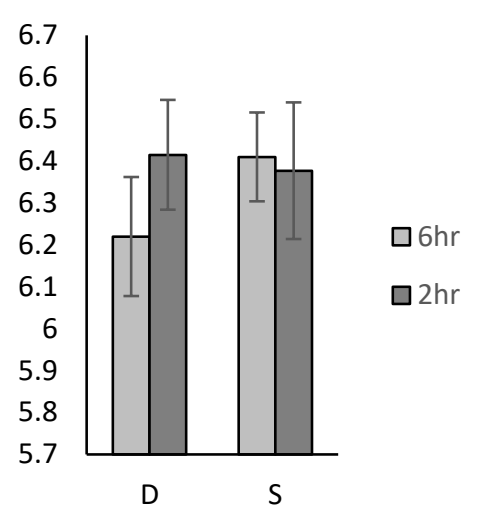

Interaction

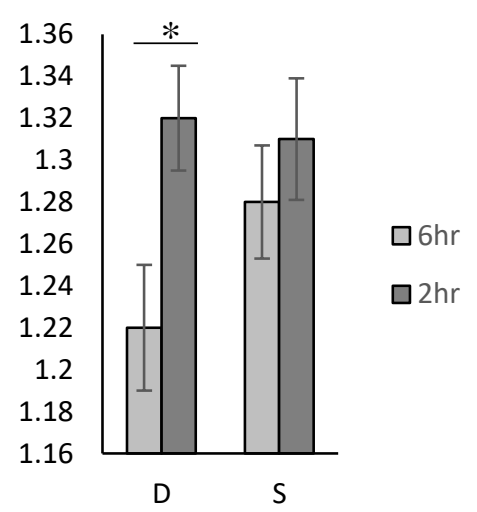

\section{Figure 3}

Body composition for Experiment 2 measured 24-hours after hatching. Hatchlings were evaluated for the effects of prenatal light duration (6hr, 2hr), prenatal light presentation (discontinuous (D), sporadic (S)), and their interaction: 6hr discontinuous (6D), 2hr discontinuous (2D), 6hr sporadic (6S), or 2hr sporadic (2S). The $6 \mathrm{hr}$ treatment is illustrated in light gray and the $2 \mathrm{hr}$ treatment is illustrated in dark gray. A: Body mass measured in grams B:

Tarsometatarsus (TARS) length measured in centimeters. * $\mathrm{p}<.05$, vertical lines represent SE. 


\section{Experiment 2: Behavior Measurements}

\section{A. Light Duration}

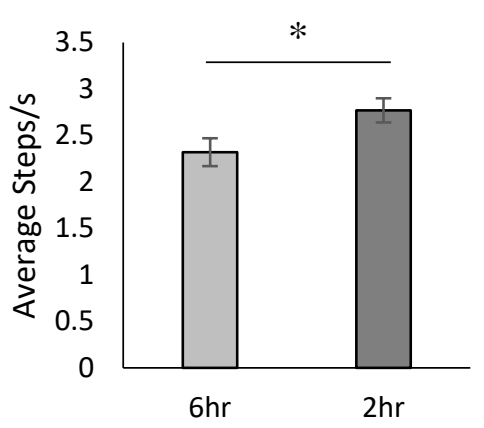

B.

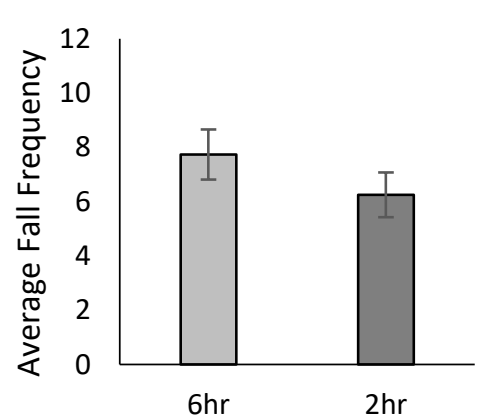

Light Presentation

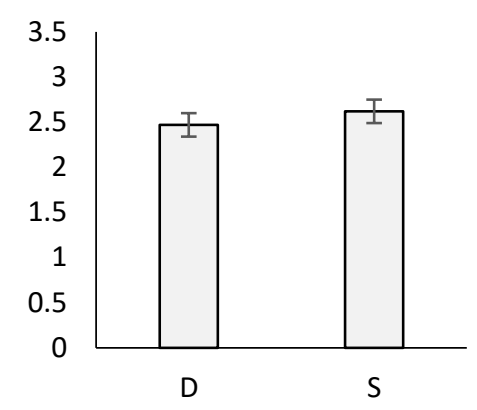

Light Presentation

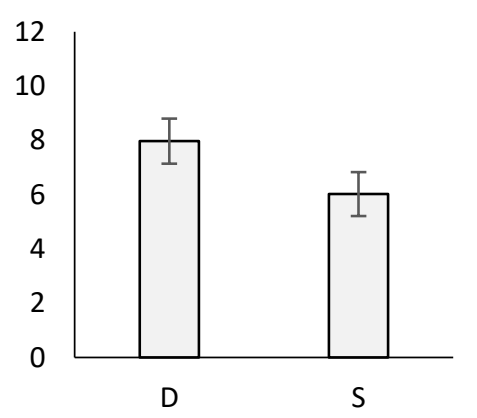

Interaction

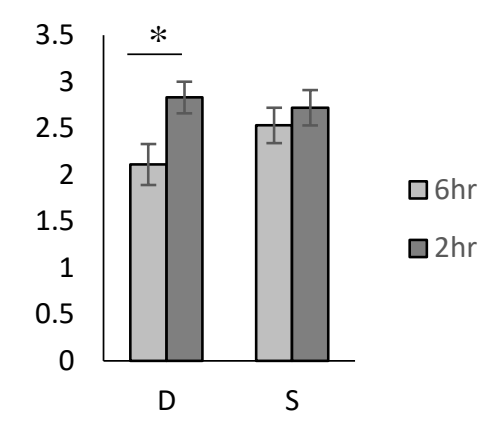

Interaction

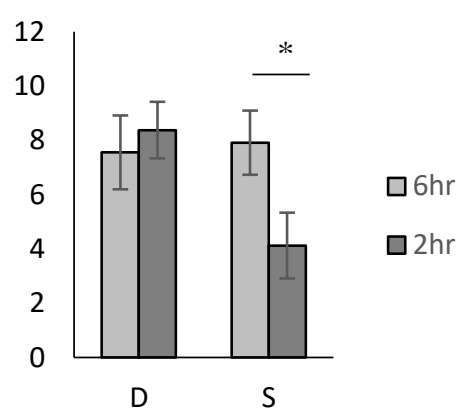

Figure 4

Behavioral measurements for experiment 2 tested at 24-hours after hatching. All values account for differences in body composition, velocity and developmental age. Chicks who experienced $2 \mathrm{hr}$ of prenatal light are illustrated in dark gray. Chicks who experienced $6 \mathrm{hr}$ of prenatal light are illustrated in light gray. The first column depicts the main effect of light duration. The second column depicts the main effect of light presentation. The third column illustrates the interaction between light duration and light presentation. A: Step cadence the average number of steps taken per second of activity. B: Fall Frequency represents the number of falls per video. Vertical lines depict SE. *p<.01 


\section{CONCLUSION}

In this series of studies, we used gait kinematics, body composition, and incubation period to evaluate the effect of prenatal temperature, prenatal movement, and prenatal light exposure on motor performance and development in bobwhite quail chicks. From these studies we can conclude that these key factors, regulated by the maternal hen, do influence motor development and growth. Specifically, we found that variations from optimal prenatal sensory conditions resulted generally in declined motor performance and augmented growth; however, each augmented treatment showed different gait perturbations and growth. For example, in Study 1 we found that cool prenatal conditions reduced body mass, increased ITJ angle and stride variability which when combined likely produced the observed increase in fall frequency; whereas in Study 2, reduced prenatal egg turning resulted in a reduction in body mass, increased gait speed and decreased stride length, but the combination of these factors did not increase the fall frequency. These different patterns suggest that different biological mechanisms are affected by domain-specific stimulation.

Understanding more about these mechanisms may provide insight into different therapeutic techniques which promote optimal motor development and in turn alleviate deficits in closely associated domains such as perception, cognition, and sociality. For example a recent study which investigated sunlight and vitamin D supplementation in depressive mother and 2-day old infants dyads found that infant exposed to sunlight showed improved motor function and reduced cortisol levels compared to vitamin D supplement treatment only and mothers exposed to sunlight reported a reduction in 
depression symptoms (Zhang et al., 2019). In Study 4, we found that the pattern of light exposure was important for bobwhite quail; quail who experienced short burst of light throughout the day mimicking the hen's nesting behavior showed improved motor function. However, we did not evaluate its effect on avian stress hormones. Nor, did we use naturally occurring sunlight. Further research is needed to see if similar results can be reproduced in quail embryos. This line of work makes it possible to evaluate specific endocrine pathways which may be influenced by sunlight and further evaluate their connection to motor systems, thereby expanding our knowledge and facilitating the development of new treatments.

Indeed, it is possible the neuroendocrine mechanistic pathways may explain most of the observed differences in motor disfunction and growth. Chicken research investigating incubation temperature (Debonne et al., 2008; Parma \& de Marchena, 2016) light (Lin Zhang et al., 2014) and turning (Tona, Onagbesan, Bruggeman, Mertens, \& Decuypere, 2005; Tona, Onagbesan, De Ketelaere, Decuypere, \& Bruggeman, 2003) indicate a connection to endocrine regulatory processes, with overlap in thyroid and stress hormones. However, it remains unclear if these processes correspond to declines in motor dysfunction. Additional research is necessary to investigate the role of neuroendocrine hormones and its relationship to maternally regulated environmental stimulation on the developing motor system.

Regardless of the mechanistic pathway, this series of studies adds corroborative evidence supporting the importance of the prenatal experiences for phenotype development. During the prenatal period many biological and neurological processes are 
being formed which play a critical role in establishing physiological and behavioral preferences. While these studies did not specifically evaluate physiological or behavioral preferences, they did show that small variations in sensory exposure during the prenatal period can negatively affect postnatal outcomes. Similarly, there is mounting evidence that environmental exposure during the prenatal period and immediately after birth are critical for determining life-long health and disease (Lieberman et al., 2000; Marques, Bjørke-Monsen, Teixeira, \& Silverman, 2015; Vallès \& Francino, 2018). Therefore, identifying which factors are important for optimal developmental is of critical value. Here we identified three non-obvious factors which are important for growth and motor performance in bobwhite quail, namely: prenatal temperature, prenatal movement, and prenatal light exposure. While it is not possible to directly compare human and animal work, our findings can help identify similar functioning processes and minimize the amount of exploratory human research necessary to discover viable treatments and develop preventative strategies to improve health and reduce disease risk (Gottlieb \& Lickliter, 2004). Further research investigating these maternally regulated factors may help to identify potential motor treatments and aid in guiding prenatal maternal care. 


\section{REFERENCES}

Abourachid, A. (2001). Kinematic parameters of terrestrial locomotion in cursorial (ratites), swimming (ducks), and striding birds (quail and guinea fowl). Comparative Biochemistry and Physiology Part A: Molecular \& Integrative Physiology, 131(1), 113-119. https://doi.org/10.1016/S1095-6433(01)00471-8

Abourachid, A., Hackert, R., Herbin, M., Libourel, P. A., Lambert, F., Gioanni, H., ... Hugel, V. (2011). Bird terrestrial locomotion as revealed by 3D kinematics. Zoology, 114(6), 360-368. https://doi.org/10.1016/j.zool.2011.07.002

Adkins, D. L., Boychuk, J., Remple, M. S., \& Kleim, J. A. (2006). Motor training induces experience-specific patterns of plasticity across motor cortex and spinal cord. Journal of Applied Physiology, 101(6), 1776-1782. https://doi.org/10.1152/japplphysiol.00515.2006

Adolph, K. E., \& Berger, S. E. (2006). Motor development. In W. Damon, R. Lerner, D. Kuhn, \& R. S. Siegler (Eds.), Handbook of child psychology: Vol. 2: Cognition, perception, and language (6th ed., pp. 161-213). Hoboken, NJ, USA: John Wiley \& Sons, Inc. https://doi.org/10.1002/9780470147658.chpsy0204

Adolph, K. E., \& Kretch, K. S. (2015). Gibson's theory of perceptual learning. In International Encyclopedia of the Social \& Behavioral Sciences (pp. 127-134). Elsevier. https://doi.org/10.1016/B978-0-08-097086-8.23096-1

Ainsworth, S. J., Stanley, R. L., \& Evans, D. J. R. R. (2010). Developmental stages of the Japanese quail. Journal of Anatomy, 216(1), 3-15. https://doi.org/10.1111/j.14697580.2009.01173.x

Akasaka, K., Nasu, T., Katayama, T., \& Murakami, N. (1995). Development of regulation of melatonin release in pineal cells in chick embryo. Brain Research, 692(1), 283-286. https://doi.org/10.1016/0006-8993(95)00643-5

Andrada, E., Nyakatura, J. A., Bergmann, F., \& Blickhan, R. (2013). Adjustments of global and local hindlimb properties during terrestrial locomotion of the common quail (Coturnix coturnix). The Journal of Experimental Biology, 216(Pt 20), 39063916. https://doi.org/10.1242/jeb.085399

Andrada, E., Nyakatura, J. A., Hochstein, S., Blickhan, R., Science, M., Jena, F., \& Jena, F. (n.d.). Terrestrial locomotion of the common quail : one leg function for all gaits?

Andrada, E., Rode, C., Sutedja, Y., Nyakatura, J. A., \& Blickhan, R. (2014). Trunk orientation causes asymmetries in leg function in small bird terrestrial locomotion. Proceedings. Biological Sciences / The Royal Society, 281(1797), 20141405-. https://doi.org/10.1098/rspb.2014.1405 
Archer, G. S., \& Mench, J. A. (2017). Exposing avian embryos to light affects post-hatch anti-predator fear responses. Applied Animal Behaviour Science, 186, 80-84. https://doi.org/10.1016/J.APPLANIM.2016.10.014

Babiker, E. M., \& Baggott, G. K. (1992). Effect of turning upon the sub-embryonic fluid and albumen of the egg of the Japanese quail. British Poultry Science, 33(5), 973991. https://doi.org/10.1080/00071669208417541

Baggott, G. K., Deeming, D. C., \& Latter, G. V. (2008). Electrolyte and Water Balance of the Early Avian Embryo: Effects of Egg Turning. Avian and Poultry Biology Reviews, 13(2), 105-119. https://doi.org/10.3184/147020602783698430

Baumgart, S. (2008). Iatrogenic hyperthermia and hypothermia in the neonate. Clinics in Perinatology, 35(1), 183-197.

Belanto, J. J., Diaz-Perez, S. V, Magyar, C. E., Maxwell, M. M., Yilmaz, Y., Topp, K., ... Jamieson, C. A. M. (2010). Dexamethasone induces dysferlin in myoblasts and enhances their myogenic differentiation. Neuromuscular Disorders : NMD, 20(2), 111-121. https://doi.org/10.1016/j.nmd.2009.12.003

Belnap, S. C., Currea, J. P., \& Lickliter, R. (2019). Prenatal incubation temperature affects neonatal precocial birds' locomotor behavior. Physiology \& Behavior, 206(1), 51-58. https://doi.org/10.1016/J.PHYSBEH.2019.03.002

Belnap, S. C., \& Lickliter, R. (2017). Coordinated movement is influenced by prenatal light experience in bobwhite quail chicks (Colinus virginianus). Behavioural Brain Research, 327, 103-111. https://doi.org/10.1016/j.bbr.2017.03.039

Biewener, A. A., \& Daley, M. A. (2007). Unsteady locomotion: integrating muscle function with whole body dynamics and neuromuscular control. Journal of Experimental Biology, 210(17), 2949-2960.

Blair, C., \& Raver, C. C. (2012). Child development in the context of adversity: experiential canalization of brain and behavior. American Psychologist, 67(4), 309318. https://doi.org/10.1037/a0027493

Boleli, I., Morita, V., Matos Jr, J., Thimotheo, M., \& Almeida, V. (2017). Poultry egg incubation: Integrating and optimizing production efficiency. Revista Brasileira de Ciência Avícola, 18(spe2), 1-16. https://doi.org/10.1590/1806-9061-2016-0292

Booth, D. T. (2006). Influence of incubation temperature on hatchling phenotype in reptiles. Physiological and Biochemical Zoology, 79(2), 274-281. https://doi.org/10.1086/499988

Booth, D. T. (2018). Incubation temperature induced phenotypic plasticity in oviparous reptiles: Where to next? J. Exp. Zool, 329, 343-350. 
https://doi.org/10.1002/jez.2195

Bradley, N. S., \& Jahng, D. Y. (2003). Selective effects of light exposure on distribution of motility in the chick embryo at E18. Journal of Neurophysiology, 90(3), 14081417. https://doi.org/10.1152/jn.00393.2003

Bradley, N. S., Solanki, D., \& Zhao, D. (2005). Limb movements during embryonic development in the chick: Evidence for a continuum in limb motor control antecedent to locomotion. Journal of Neurophysiology. https://doi.org/10.1152/jn.00804.2005

Brown, S. A., Zumbrunn, G., Fleury-Olela, F., Preitner, N., \& Schibler, U. (2002). Rhythms of mammalian body temperature can sustain peripheral circadian clocks. Current Biology, 12(18), 1574-1583. https://doi.org/10.1016/S09609822(02)01145-4

Bushnell, E. W., \& Boudreau, J. P. (1993). Motor development and the mind: The potential role of motor abilities as a determinant of aspects of perceptual development. Child Development, 64(4), 1005-1021. https://doi.org/10.1111/j.14678624.1993.tb04184.x

Carlsen, R., \& Lickliter, R. (1999). Augmented prenatal tactile and vestibular stimulation alters postnatal auditory and visual responsiveness in bobwhite quail chicks. Developmental Psychobiology, 35(3), 215-225. https://doi.org/10.1002/(SICI)10982302(199911)35:3<215::AID-DEV6>3.0.CO;2-O

Carroll, R. L., Davis, C. A., Fuhlendorf, S. D., Elmore, R. D., DuRant, S. E., \& Carroll, J. M. (2018). Avian parental behavior and nest success influenced by temperature fluctuations. Journal of Thermal Biology, 74, 140-148. https://doi.org/10.1016/J.JTHERBIO.2018.03.020

Casey, M. B., \& Sleigh, M. J. (2014). Prenatal visual experience induces postnatal motor laterality in japanese quail chicks (Coturnix coturnix japonica). Developmental Psychobiology, 56, 489-497. https://doi.org/10.1002/dev.21116

Catama, B. V, Calalang, M. S., Cada, R., Karlo, D., Ballog, A. C., Batton, K. B., ... Jan, J. (2017). Motor intervention activities for children with autism spectrum disorders. International Journal of Research Studies in Psychology, 6(1), 27-42. https://doi.org/10.5861/ijrsp.2017.1667

Chiandetti, C., Galliussi, J., Andrew, R. J., \& Vallortigara, G. (2013). Early-light embryonic stimulation suggests a second route, via gene activation, to cerebral lateralization in vertebrates. Scientific Reports, 3(1), 2701. https://doi.org/10.1038/srep02701 
Chin, E. H., Love, O. P., Verspoor, J. J., Williams, T. D., Rowley, K., \& Burness, G. (2009). Juveniles exposed to embryonic corticosterone have enhanced flight performance. Proceedings of the Royal Society B: Biological Sciences, 276(1656), 499-505. https://doi.org/10.1098/rspb.2008.1294

Clapp, J.F. (2006). Influence of endurance exercise and diet on human placental development and fetal growth. Placenta, 27(6-7), 527-534.

https://doi.org/10.1016/J.PLACENTA.2005.07.010

Clapp, James F., \& Rizk, K. H. (1992). Effect of recreational exercise on midtrimester placental growth. American Journal of Obstetrics and Gynecology, 167(6), 15181521. https://doi.org/10.1016/0002-9378(92)91730-X

Clark, D. L., Coy, C. S., Strasburg, G. M., Reed, K. M., \& Velleman, S. G. (2016). Temperature effect on proliferation and differentiation of satellite cells from Turkeys with different growth rates. Poultry Science. https://doi.org/10.3382/ps/pev437

Clearfield, M. W., Osborne, C. N., \& Mullen, M. (2008). Learning by looking: Infants' social looking behavior across the transition from crawling to walking. Journal of Experimental Child Psychology, 100(4), 297-307. https://doi.org/10.1016/j.jecp.2008.03.005

Cockburn, A. (2006). Prevalence of different modes of parental care in birds. Proceedings. Biological Sciences, 273(1592), 1375-1383. https://doi.org/10.1098/rspb.2005.3458

Cohen, J. (1988). The effect size index: d. In Statistical Power Analysis for the Behavioral Sciences (2nd ed., pp. 20-26). Psychology Press.

Collias, N. E., \& Eollias, E. C. (1984). Nest Building and Bird Behavior - Nicholas E. Collias, Elsie C. Collias - Google Books. New Jersey: Princeton University Press.

Collin, A., Berri, C., Tesseraud, S., Rodon, F. E. R., Skiba-Cassy, S., Crochet, S., ... Yahav, S. (2007). Effects of thermal manipulation during early and late embryogenesis on thermotolerance and breast muscle characteristics in broiler chickens. Poultry Science, 86(5), 795-800. https://doi.org/10.1093/ps/86.5.795

Cook, J. (2016). From movement kinematics to social cognition: the case of autism. Philosophical Transactions of the Royal Society B: Biological Sciences, 371(1693), 20150372. https://doi.org/10.1098/rstb.2015.0372

Cooke, S. J., Schreer, J. F., Philipp, D. P., \& Weatherhead, P. J. (2003). Nesting activity, parental care behavior, and reproductive success of smallmouth bass, Micropterus dolomieu, in an unstable thermal environment. Journal of Thermal Biology, 28(6-7), 445-456. https://doi.org/10.1016/S0306-4565(03)00038-X 
Cooper, C. B., Voss, M. A., Ardia, D. R., Austin, S. H., \& Robinson, W. D. (2011). Light increases the rate of embryonic development: Implications for latitudinal trends in incubation period. Functional Ecology, 25(4), 769-776.

https://doi.org/10.1111/j.1365-2435.2011.01847.x

Datavyu Team. (2014). Datavyu: A video coding tool. Databrary Project.

de Vries, J. I. P., Visser, G. H. A., \& Prechtl, H. F. R. (1982). The emergence of fetal behaviour. I. Qualitative aspects. Early Human Development, 7(4), 301-322. https://doi.org/10.1016/0378-3782(82)90033-0

Deaton, K. E., Bishop, C. M., \& Butler, P. J. (1997). The effect of thyroid hormones on the aerobic development of locomotor and cardiac muscles in the barnacle goose. Journal of Comparative Physiology B: Biochemical, Systemic, and Environmental Physiology, 167(5), 319-327. https://doi.org/10.1007/s003600050080

Debonne, M., Baarendse, P. J. J., Van Den Brand, H., Kemp, B., Bruggeman, V., \& Decuypere, E. (2008). Involvement of the hypothalamic-pituitary-thyroid axis and its interaction with the hypothalamic-pituitary-adrenal axis in the ontogeny of avian thermoregulation: A review. World's Poultry Science Journal. https://doi.org/10.1017/S0043933908000056

Deeming, D. C. (1989). Failure to turn eggs during incubation: Development of the area vasculosa and embryonic growth. Journal of Morphology, 201(2), 179-186. https://doi.org/10.1002/jmor.1052010207

Deeming, D. C. (2009). The role of egg turning during incubation. Avian Biology Research, 2(1-2), 67-71. https://doi.org/10.3184/175815509X431849

Deeming, D. C., \& Ferguson, M. (1992). Egg Incubation - Its Effects On EmbryonicDevelopment In Birds And Reptiles. Cambrige University Press. https://doi.org/10.1017/CBO9780511585739

Diamond, A. (2000). Close interrelation of motor development and cognitive development and of the cerebellum and prefrontal cortex. Child Development, 71(1), 44-56. https://doi.org/10.1111/1467-8624.00117

Dickman, J. D., \& Lim, I. (2004). Posture, head stability, and orientation recovery during vestibular regeneration in pigeons. Journal of the Association for Research in Otolaryngology, 5(3), 323-336. https://doi.org/10.1007/s10162-004-4047-0

Dong, Y., Pan, J. S., \& Zhang, L. (2013). Myostatin Suppression of Akirin1 Mediates Glucocorticoid-Induced Satellite Cell Dysfunction. PLoS ONE, 8(3), e58554. https://doi.org/10.1371/journal.pone.0058554

Du, W.-G., \& Ji, X. (2003). The effects of incubation thermal environments on size, locomotor performance and early growth of hatchling soft-shelled turtles, Pelodiscus 
sinensis. Journal of Thermal Biology, 28, 279-286. https://doi.org/10.1016/S03064565(03)00003-2

DuRant, S. E., Carter, A. W., Denver, R. J., Hepp, G. R., \& Hopkins, W. A. (2014). Are thyroid hormones mediators of incubation temperature-induced phenotypes in birds?, 10(1), 20130950. https://doi.org/10.1098/rsbl.2013.0950

DuRant, S. E., Hepp, G. R., Moore, I. T., Hopkins, B. C., \& Hopkins, W. A. (2010). Slight differences in incubation temperature affect early growth and stress endocrinology of wood duck (Aix sponsa) ducklings. The Journal of Experimental Biology, 213(1), 45-51. https://doi.org/10.1242/jeb.034488

DuRant, S. E., Hopkins, W. A., Carter, A. W., Stachowiak, C. M., \& Hepp, G. R. (2013). Incubation conditions are more important in determining early thermoregulatory ability than posthatch resource conditions in a precocial bird. Physiological and Biochemical Zoology, 86(4), 410-420. https://doi.org/10.1086/671128

DuRant, S. E., Hopkins, W. A., Hepp, G. R., \& Walters, J. R. (2013). Ecological, evolutionary, and conservation implications of incubation temperature-dependent phenotypes in birds. Biological Reviews, 88(2), 499-509. https://doi.org/10.1111/brv.12015

Durlak, J. A. (2009). How to select, calculate, and interpret effect sizes. Journal of Pediatric Psychology. https://doi.org/10.1093/jpepsy/jsp004

Dwyer, C., Madgwick, A., Ward, S., \& Stickland, N. (1995). Effect of maternal undernutrition in early gestation on the development of fetal myofibres in the guinea-pig. Reproduction, Fertility and Development, 7(5), 1285. https://doi.org/10.1071/RD9951285

Ebersbach, G., Sojer, M., Valldeoriola, F., Wissel, J., Müller, J., Tolosa, E., \& Poewe, W. (1999). Comparative analysis of gait in Parkinson's disease, cerebellar ataxia and subcortical arteriosclerotic encephalopathy. Brain, 122(7), 1349-1355. https://doi.org/10.1093/brain/122.7.1349

Edwards, M. J. (1986). Hyperthermia as a teratogen: A review of experimental studies and their clinical significance. Carcinogenesis, and Mutagenesis, 6563-6582.

Edwards, Marshall J. (2006). Review: Hyperthermia and fever during pregnancy. Birth Defects Research Part A: Clinical and Molecular Teratology, 76(7), 507-516. https://doi.org/10.1002/bdra.20277

Edwards, Marshall J., Shiota, K., Smith, M. S. R., \& Walsh, D. A. (1995, September 1). Hyperthermia and birth defects. Reproductive Toxicology. Pergamon. https://doi.org/10.1016/0890-6238(95)00043-A

Eiby, Y. A., Wilmer, J. W., \& Booth, D. T. (2008). Temperature-dependent sex-biased 
embryo mortality in a bird. Proceedings. Biological Sciences, 275(1652), 27032706. https://doi.org/10.1098/rspb.2008.0954

Elphick, M. J., \& Shine, R. (1998). Longterm effects of incubation temperatures on the morphology and locomotor performance of hatchling lizards. Biological Journal of the Linnean Society, 63(3), 429-447. https://doi.org/10.1111/j.10958312.1998.tb01527.x

Everaert, N., Métayer-Coustard, S., Willemsen, H., Han, H., Song, Z., Ansari, Z., ... Tesseraud, S. (2013). The effect of albumen removal before incubation (embryonic protein under-nutrition) on the post-hatch performance, regulators of protein translation activation and proteolysis in neonatal broilers. British Journal of Nutrition, 110(2), 265-274. https://doi.org/10.1017/S000711451200503X

Fermin, C. D., Martin, D., Jones, T. A., Vellinger, J. C., Deuser, M., Hester, P., \& Hullinger, R. (1996). Microgravity in the STS-29 space shuttle discovery affected the vestibular system of chick embryos. Histology and Histopathology, 11, 407-426.

Field, T., Diego, M., \& Hernandez-Reif, M. (2010). Preterm infant massage therapy research: A review. Infant Behavior and Development. https://doi.org/10.1016/j.infbeh.2009.12.004

French, N. A. (1997). Modeling incubation temperature: the effects of incubator design, embryonic development, and egg size. Poultry Science, 76(1), 124-133. https://doi.org/10.1093/ps/76.1.124

Gardahaut, M. F., Fontaine-Perus, J., Rouaud, T., Bandman, E., \& Ferrand, R. (1992). Developmental modulation of myosin expression by thyroid hormone in avian skeletal muscle. Development, 115(4).

Gatesy, S. M., \& Biewener, A. A. (1991). Bipedal locomotion: effects of speed, size and limb posture in birds and humans. Journal of Zoology, 224(1), 127-147. https://doi.org/10.1111/j.1469-7998.1991.tb04794.x

Geisler, H. C., \& Gramsbergen, A. (1998). Motor development after vestibular deprivation in rats. Neuroscience and Biobehavioral Reviews. https://doi.org/10.1016/S0149-7634(97)00045-6

Gibson, J. J. (1979). Gibson theory of affordances. In J. J. Gieseking, W. Mangold, C. Katz, S. Low, \& S. Saegert (Eds.), The People, Place, and Space Reader (pp. 5660). Routledge. https://doi.org/citeulike-article-id:3508530

Gilbert, M. E., Hedge, J. M., Valentín-Blasini, L., Blount, B. C., Kannan, K., Tietge, J., ... Fisher, J. W. (2013). An animal model of marginal iodine deficiency during development: The thyroid axis and neurodevelopmental outcome. Toxicological Sciences. https://doi.org/10.1093/toxsci/kfs335 
Gimeno, M. A., Roberts, C. M., \& Webb, J. L. (1967). Acceleration of rate of the early chick embryo heart by visible light. Nature, 214(5092), 1014-1016. https://doi.org/10.1038/2141014a0

Ginzberg, R. D., \& Gilula, N. B. (1980). Synaptogene sis in the vestibular sensory epithelium of the chick embryo. Journal of Neurocytology, 9(3), 405-424. https://doi.org/10.1007/BF01181545

Glover, J. C. (2003). The development of vestibulo-ocular circuitry in the chicken embryo. Journal of Physiology-Paris, 97(1), 17-25. https://doi.org/10.1016/J.JPHYSPARIS.2003.10.003

Godfrey, K., Robinson, S., Barker, D. J., Osmond, C., \& Cox, V. (1996). Maternal nutrition in early and late pregnancy in relation to placental and fetal growth. $B M J$ (Clinical Research Ed.), 312(7028), 410-414. https://doi.org/10.1136/bmj.312.7028.410

Gottesman-Davis, A. (2011). Structure-function studies of second-order vestibular nucleus neurons in the developing chicken. George Washington University.

Gottlieb, G. (1968). Prenatal behavior of birds. In The Quarterly Review of Biology (Vol. 43, pp. 148-174).

Gottlieb, G. (1971). Ontogenesis of sensory function in birds and mammals. (E. Tobach, L. Aronson, \& E. Shaw, Eds.), The Biopsychology of Development. New York: Academic Press.

Gottlieb, G., \& Lickliter, R. (2004). The various roles of animal models in understanding human development. Social Development, 13, 311-325.

Gottlieb, G., Tomlinson, W. T., \& Radell, P. L. (1989). Developmental intersensory interference: Premature visual experience suppresses auditory learning in ducklings. Infant Behavior and Development, 12(1), 1-12. https://doi.org/10.1016/01636383(89)90048-9

Greeno, J. G. (1994). Gibson's affordances. Psychological Review, 101(2), 336-342. https://doi.org/10.1037/0033-295X.101.2.336

Guderley, H. (2004). Locomotor performance and muscle metabolic capacities: impact of temperature and energetic status. Comparative Biochemistry and Physiology Part B: Biochemistry and Molecular Biology, 139(3), 371-382. https://doi.org/10.1016/J.CBPC.2004.04.001

Guenther, A., \& Trillmich, F. (2013). Photoperiod influences the behavioral and physiological phenotype during ontogeny. Behavioral Ecology, 24(2), 402-411. https://doi.org/10.1093/beheco/ars177 
Hall, B. K., \& Herring, S. W. (1990). Paralysis and growth of the musculoskeletal system in the embryonic chick. Journal of Morphology, 206(1), 45-56.

https://doi.org/10.1002/jmor.1052060105

Hamburger, V. (1973). Anatomical and physiological basis of embryonic motility in birds and mammals. (G. Gottlieb, Ed.), Behavioral Embryology (Vol. 1). Boston, MA: Birkhäuser Boston. https://doi.org/10.1007/978-1-4899-6743-5_12

Hamburger, V., \& Balaban, M. (1963). Observations and experiments on spontaneous rhythmical behavior in the chick embryo. Developmental Biology, 7, 533-545. https://doi.org/10.1016/0012-1606(63)90140-4

Hamburger, V., \& Hamilton, H. L. (1951). A series of normal stages in the development of the chick embryo. Journal of Morphology, 88(1), 49-92.

https://doi.org/10.1002/jmor.1050880104

Hammond, C. L., Simbi, B. H., \& Stickland, N. C. (2007). In ovo temperature manipulation influences embryonic motility and growth of limb tissues in the chick (Gallus gallus). The Journal of Experimental Biology, 210(15), 2667-2675. https://doi.org/10.1242/jeb.005751

Han, V. K., \& Carter, A. M. (2001). Control of growth and development of the fetoplacental unit. Current Opinion in Pharmacology, 1, 632-640. Retrieved from https://heinz.sdu.dk:8443/ws/files/47387608/01_03_COP_Review.pdf

Hausdorff, J. M. (2005). Gait variability: methods, modeling and meaning. Journal of NeuroEngineering and Rehabilitation, 2(1), 19. https://doi.org/10.1186/1743-00032-19

Hausdorff, J. M. (2007). Gait dynamics, fractals and falls: Finding meaning in the strideto-stride fluctuations of human walking. Human Movement Science, 26(4), 555-589. https://doi.org/10.1016/j.humov.2007.05.003

Hendrickx, A. G., \& Hanzlik, R. (1965). Developmental stages of the bob-white quail embryo (Colinus virginianus). The Biological Bulletin, 129(3), 523-531. https://doi.org/10.2307/1539730

Hepp, G. R., Kennamer, R. A., \& Johnson, M. H. (2006). Maternal effects in Wood Ducks: Incubation temperature influences incubation period and neonate phenotype. Functional Ecology, 20(2), 307-314. https://doi.org/10.1111/j.13652435.2006.01108.x

Hepper, P. G. (2015). Behavior during the prenatal period: Adaptive for development and survival. Child Development Perspectives, 9(1), 38-43. https://doi.org/10.1111/cdep.12104

Higgins, L., Greenwood, S. L., Wareing, M., Sibley, C. P., \& Mills, T. A. (2011). Obesity 
and the placenta: A consideration of nutrient exchange mechanisms in relation to aberrant fetal growth. Placenta, 32(1), 1-7.

https://doi.org/10.1016/J.PLACENTA.2010.09.019

Hill, W. L. (1993). Importance of prenatal nutrition to the development of a precocial chick. Developmental Psychobiology, 26(5), 237-249.

https://doi.org/10.1002/dev.420260502

Honeycutt, H., \& Lickliter, R. (2003). The Influence of prenatal tactile and vestibular stimulation on auditory and visual responsiveness in bobwhite quail: A matter of timing. Developmental Psychobiology, 43, 73-81.

Hope, S. F., Kennamer, R. A., Moore, I. T., \& Hopkins, W. A. (2018). Incubation temperature influences the behavioral traits of a young precocial bird. Journal of Experimental Zoology Part A: Ecological and Integrative Physiology, 329(4-5), 191-202. https://doi.org/10.1002/jez.2176

Hopkins, B. C., Durant, S. E., Hepp, G. R., \& Hopkins, W. A. (2011). Incubation temperature influences locomotor performance in young wood ducks (Aix sponsa). Journal of Experimental Zoology Part A: Ecological Genetics and Physiology, 315 A(5), 274-279. https://doi.org/10.1002/jez.673

Iqbal, A., Decuypere, E., Abd El Azim, A., \& Kühn, E. R. (1990). Pre- and post-hatch high temperature exposure affects the thyroid hormones and corticosterone response to acute heat stress in growing chicken (Gallus domesticus). Journal of Thermal Biology, 15(2), 149-153. https://doi.org/10.1016/0306-4565(90)90032-D

Ischer, T., Ireland, K., \& Booth, D. T. (2009). Locomotion performance of green turtle hatchlings from the Heron Island Rookery, Great Barrier Reef. Marine Biology, 156(7), 1399-1409. https://doi.org/10.1007/s00227-009-1180-7

Johnson, B. P., Lum, J. A. G., Rinehart, N. J., \& Fielding, J. (2016). Ocular motor disturbances in autism spectrum disorders: Systematic review and comprehensive meta-analysis. Neuroscience \& Biobehavioral Reviews, 69, 260-279. https://doi.org/10.1016/j.neubiorev.2016.08.007

Jones, S. M., \& Jones, T. A. (1996). Short latency vestibular evoked potentials in the chicken embryo. J. Vestib. Res.-Equilib. Orientat., 6(2), 71-83.

Kannape, O. A., \& Blanke, O. (2013). Self in motion: sensorimotor and cognitive mechanisms in gait agency. Journal of Neurophysiology, 110(8), 1837-1847. https://doi.org/10.1152/jn.01042.2012

Kardon, G. (1998). Muscle and tendon morphogenesis in the avian hind limb. Development, 125(20).

Kelso, J. A. S. (2016). On the self-organizing origins of agency. Trends in Cognitive 
Sciences, 20(7), 490-499. https://doi.org/10.1016/j.tics.2016.04.004

Kendeigh, S. C. (1952). Parental care and its evolution in birds. (Harley Cleave; H. Halvorson; William Horsfall; Leland Shanor, Ed.), Illinois biological monographs (22nd ed.). University of Illinois Press .

Kenyon, R. V, Kerschmann, R., \& Silbergleit, R. (1988). Streptomycin in the chick embryo: post-hatching vestibular behavior and morphology. Experimental Brain Research (Vol. 69). Springer-Verlag.

Khalil, H. A. (2009). Productive and physiological responses of Japanese quail embryos to light regime during incubation period. Slovak Journal of Animal Science, 42(2), $79-86$.

Kluger, M. J., Kozak, W., Conn, C. A., Leon, L. R., \& Soszynski, D. (1996). The adaptive value of fever. Infectious Disease Clinics of North America, 10(1), 1-20. https://doi.org/10.1016/S0891-5520(05)70282-8

Koch, S. C., Mehl, L., Sobanski, E., Sieber, M., \& Fuchs, T. (2015). Fixing the mirrors: A feasibility study of the effects of dance movement therapy on young adults with autism spectrum disorder. Autism, 19(3), 338-350. https://doi.org/10.1177/1362361314522353

Kolbe, J. J., \& Janzen, F. J. Impact of nest-site selection on nest success and nest temperature in natural and disturbed habitats, 83 Ecology § (2002).

Kulig, B. M. (1996). Comprehensive Neurotoxicity Assessment. Environ Health Perspect (Vol. 104).

Lan, W., Feldkaemper, M., \& Schaeffel, F. (2014). Intermittent episodes of bright light suppress myopia in the chicken more than continuous bright light. PLoS ONE, 9(10), e110906. https://doi.org/10.1371/journal.pone.0110906

Lauber, J. K. (1975). Photoacceleration of avian embryogenesis. Comparative Biochemistry and Physiology -- Part A: Physiology, 51(4), 903-907. https://doi.org/10.1016/0300-9629(75)90073-0

Le Ray, C. F., Prevette, D., Oppenheim, R. W., \& Fontaine-Perus, J. (1993). Interactions between spinal cord stimulation and activity blockade in the regulation of synaptogenesis and motoneuron survival in the chick embryo. Journal of Neurobiology, 24(9), 1142-1156. https://doi.org/10.1002/neu.480240903

Leonard, H. C. (2016). The impact of poor motor skills on perceptual, social and cognitive development: The case of developmental coordination disorder. Frontiers in Psychology, 7, 311. https://doi.org/10.3389/fpsyg.2016.00311

Leonard, H. C., \& Hill, E. L. (2014). The impact of motor development on typical and 
atypical social cognition and language: A systematic review. Child and Adolescent Mental Health, 19(3), 163-170. https://doi.org/10.1111/camh.12055

Lickliter, R. (2005). Prenatal sensory ecology and experience: Implications for perceptual and behavioral development in precocial birds. In Advances in the Study of Behavior (pp. 235-274). Elsevier. https://doi.org/10.1016/S0065-3454(05)35006-6

Lickliter, R. (2011). The integrated development of sensory organization. Clinics in Perinatology, 38(4), 591-603. https://doi.org/10.1016/j.clp.2011.08.007

Lickliter, R., Bahrick, L. E., \& Honeycutt, H. (2002). Intersensory redundancy facilitates prenatal perceptual learning in bobwhite quail (Colinus virginianus) embryos. Developmental Psychology, 38(1), 15-23.

Lieberman, E., Lang, J., Richardson, D. K., Frigoletto, F. D., Heffner, L. J., \& Cohen, A. (2000). Intrapartum maternal fever and neonatal outcome. Pediatrics, 105(1 Pt 1), 8-13. https://doi.org/10.1542/PEDS.105.1.8

Lilienthal, S., Drotleff, A. M., \& Ternes, W. (2015). Changes in the protein secondary structure of hen's egg yolk determined by Fourier transform infrared spectroscopy during the first eight days of incubation. Poultry Science, 94(1), 68-79. https://doi.org/10.3382/ps/peu051

Lloyd, M., MacDonald, M., \& Lord, C. (2013). Motor skills of toddlers with autism spectrum disorders. Autism, 17(2), 133-146. https://doi.org/10.1177/1362361311402230

Lloyd, M., Reid, G., \& Bouffard, M. (2006). Self-regulation of sport specific and educational problem-solving tasks by boys with and without DCD. Adapted Physical Activity Quarterly, 23(4), 370-389. https://doi.org/10.1123/apaq.23.4.370

Lorenzi, E., Mayer, U., Rosa-Salva, O., Morandi-Raikova, A., \& Vallortigara, G. (2019). Spontaneous and light-induced lateralization of immediate early genes expression in domestic chicks. Behavioural Brain Research, 368, 111905. https://doi.org/10.1016/J.BBR.2019.111905

Loyau, T., Berri, C., Bedrani, L., Métayer-Coustard, S., Praud, C., Duclos, M. J., ... Collin, A. (2013). Thermal manipulation of the embryo modifies the physiology and body composition of broiler chickens reared in floor pens without affecting breast meat processing quality. Journal of Animal Science. https://doi.org/10.2527/jas.2013-6445

Lyall, K., Anderson, M., Kharrazi, M., \& Windham, G. C. (2017). Neonatal thyroid hormone levels in association with autism spectrum disorder. Autism Research, 10(4), 585-592. https://doi.org/10.1002/aur.1708

Lychakov, D. V, Il'inskaia, E. V, Dadasheva, O. A., \& Gur'eva, T. S. (1993). [The 
vestibular apparatus of quail embryos in an experiment on the Kosmos-1129 biosatellite]. Aviakosmicheskaia i Ekologicheskaia Meditsina [ Aerospace and Environmental Medicine], 27(2), 68-71.

Maldonado, M. A., Allred, R. P., Felthauser, E. L., \& Jones, T. A. (2008). Motor skill training, but not voluntary exercise, improves skilled reaching after unilateral ischemic lesions of the sensorimotor cortex in rats. Neurorehabilitation and Neural Repair, 22(3), 250-261. https://doi.org/10.1177/1545968307308551

Mangwiro, Y. T., Briffa, J. F., Gravina, S., Mahizir, D., Anevska, K., Romano, T., ... Wlodek, M. E. (2018). Maternal exercise and growth restriction in rats alters placental angiogenic factors and blood space area in a sex-specific manner. Placenta, 74, 47-54. https://doi.org/10.1016/j.placenta.2018.12.005

Mariette, M. M., \& Buchanan, K. L. (2016). Prenatal acoustic communication programs offspring for high posthatching temperatures in a songbird. Science, 353(6301), 812-814. https://doi.org/10.1126/science.aaf7049

Marques, A. H., Bjørke-Monsen, A.-L., Teixeira, A. L., \& Silverman, M. N. (2015). Maternal stress, nutrition and physical activity: Impact on immune function, CNS development and psychopathology. Brain Research, 1617, 28-46. https://doi.org/10.1016/J.BRAINRES.2014.10.051

Marsh, R. L., \& Dawson, W. R. (1989). Avian adjustments to cold. In Advances in Comparative and Environmental Physiology volume 4 (pp. 205-253). Springer, Berlin, Heidelberg. https://doi.org/10.1007/978-3-642-74078-7_6

Martin, T. E., Auer, S. K., Bassar, R. D., Niklison, A. M., \& Lloyd, P. (2007). Geographic variation in avian incubation periods and parental influences on embryonic temperature. Evolution, 61(11), 2558-2569. https://doi.org/10.1111/j.1558-5646.2007.00204.x

Mills, A. D., Crawford, L. L., Domjan, M., \& Faure, J. M. (1997). The behavior of the Japanese or Domestic quail Coturnix japonica Coturnix japonica. Neuroscience and Biobehavioral Reviews, 21(3), 261-281.

Mrosovsky, N., \& Pieau, C. (1991). Transitional range of temperature, pivotal temperatures and thermosensitive stages for sex determination in reptiles. Amphibia Reptilia, 12(2), 169-179. https://doi.org/10.1163/156853891X00149

Muir, G. D. (2000). Early ontogeny of locomotor behaviour: A comparison between altricial and precocial animals. Brain Research Bulletin. https://doi.org/10.1016/S0361-9230(00)00404-4

Muir, G. D., \& Chu, T. K. (2002). Posthatching locomotor experience alters locomotor development in chicks. Journal of Neurophysiology, 88, 117-123. 
https://doi.org/10.1152/jn.00855.2001

Muir, G. D., Gosline, J. M., \& Steeves, J. D. (1996). Ontogeny of bipedal locomotion: walking and running in the chick. The Journal of Physiology, 493(2), 589-601. https://doi.org/10.1113/jphysiol.1996.sp021406

Muir, G. D., \& Steeves, J. D. (1995). Phasic cutaneous input facilitates locomotor recovery after incomplete spinal injury in the chick. Journal of Neurophysiology, 74(1), 358-368. https://doi.org/10.1152/jn.1995.74.1.358

Muller, G. B. (2003). Embryonic motility: environmental influences and evolutionary innovation. Evolution and Development, 5(1), 56-60. https://doi.org/10.1046/j.1525142X.2003.03009.x

Murray, M. P. (1967). Gait as a total pattern of movement: Including a bibliography on gait. American Jounal of Physical Medicine \& Rehabilitation, 46(1), 209-233.

Nelson, M. N., White-Traut, R. C., Vasan, U., Silvestri, J., Comiskey, E., Meleedy-Rey, P., ... Patel, M. (2001). One-year outcome of auditory-tactile-visual-vestibular intervention in the neonatal intensive care unit: Effects of Severe prematurity and central nervous system injury. Journal of Child Neurology, 16(7), 493-498.

Nesan, D., Kamkar, M., Burrows, J., Scott, I. C., Marsden, M., \& Vijayan, M. M. (2012). Glucocorticoid receptor signaling is essential for mesoderm formation and muscle development in zebrafish. Endocrinology, 153(3), 1288-1300. https://doi.org/10.1210/en.2011-1559

Nickel, L. R., Thatcher, A. R., Keller, F., Wozniak, R. H., \& Iverson, J. M. (2013). Posture development in infants at heightened versus low risk for autism spectrum disorders. Infancy, 18(5), 639-661. https://doi.org/10.1111/infa.12025

Nowlan, N. C., Sharpe, J., Roddy, K. A., Prendergast, P. J., \& Murphy, P. (2010). Mechanobiology of embryonic skeletal development: Insights from animal models. Birth Defects Research. Part C, Embryo Today: Reviews, 90(3), 203-213. https://doi.org/10.1002/bdrc.20184

Nudds, R. L., Folkow, L. P., Lees, J. J., Tickle, P. G., Stokkan, K.-A., \& Codd, J. R. (2011). Evidence for energy savings from aerial running in the svalbard rock ptarmigan (Lagopus muta hyperborea). Proceedings of the Royal Society of London B: Biological Sciences, 278(1718).

Nyakatura, J. A., Andrada, E., Dunlap, K., Mowrer, O., Friedman, M., Frost, B., ... Full, R. (2014). On vision in birds: coordination of head-bobbing and gait stabilises vertical head position in quail. Frontiers in Zoology, 11(1), 27. https://doi.org/10.1186/1742-9994-11-27

Ocklenburg, S., \& Güntürkün, O. (2012). Hemispheric asymmetries: The comparative 
view. Frontiers in Psychology, 3(January), 1-9.

https://doi.org/10.3389/fpsyg.2012.00005

Olanrewaju, H. A., Thaxton, J. P., Dozier, W. A., Purswell, J., Roush, W. B., \& Branton, S. L. (2006). A review of lighting programs for broiler production. International Journal of Poultry Science, 5(4), 301-308.

Oliveira, A. O., Fileto, C., \& Melis, M. S. (2004). Effect of strenuous maternal exercise before and during pregnancy on rat progeny renal function. Brazilian Journal of Medical and Biological Research, 37(6), 907-911. https://doi.org/10.1590/S0100879X2004000600017

Olsen, M. (1930). Influence of turning and other factors on the hatching power of hens eggs... Iowa State College.

Olson, C. R., Vleck, C. M., \& Vleck, D. (2006). Periodic cooling of bird eggs reduces embryonic growth efficiency. Physiological and Biochemical Zoology, 79(5), 927936. https://doi.org/10.1086/506003

Oppenheim, R. W. (1972). Prehatching and hatching behaviour in birds: A comparative study of altricial and precocial species. Animal Behaviour, 20(4), 644-655. https://doi.org/10.1016/S0003-3472(72)80137-4

Orcutt, F. . S. J., \& Orcutt, A. B. . (1976). Nesting and parental behavior in domestic common quail. The Auk, 93(1), 135-141. Retrieved from http://www.jstor.org/stable/4084840

Özkan, S., Yalçin, S., Babacanoğlu, E., Kozanoğlu, H., Karadaş, F., \& Uysal, S. (2012). Photoperiodic lighting (16 hours of light:8 hours of dark) programs during incubation: 1. Effects on growth and circadian physiological traits of embryos and early stress response of broiler chickens. Poultry Science, 91(11), 2912-2921. https://doi.org/10.3382/ps.2012-02426

Parma, V., \& de Marchena, A. B. (2016). Motor signatures in autism spectrum disorder: the importance of variability. Journal of Neurophysiology, 115(3). https://doi.org/10.1152/jn.00647.2015

Pearson, K. G. (2004). Generating the walking gait: role of sensory feedback. Progress in Brain Research, 143, 123-129. https://doi.org/10.1016/S0079-6123(03)43012-4

Pereira, R. M. R., \& Freire de Carvalho, J. (2011). Glucocorticoid-induced myopathy. Joint Bone Spine, 78(1), 41-44. https://doi.org/10.1016/J.JBSPIN.2010.02.025

Pettersson, A. F., Olsson, E., \& Wahlund, L.-O. (2005). Motor function in subjects with mild cognitive impairment and early Alzheimer's Disease. Dementia and Geriatric Cognitive Disorders, 19(5-6), 299-304. https://doi.org/10.1159/000084555 
Peusner, K. D., \& Giaume, C. (1997). Ontogeny of electrophysiological properties and dendritic pattern in second-order chick vestibular neurons. THE JOURNAL OF COMPARATIVE NEUROLOGY (Vol. 384). Wiley-Liss, Inc.

Piaget, J. (1951). Principal factors determlning intellectual evolution from childhood to adult life. In Organization and pathology of thought: Selected sources. (pp. 154175). New York: Columbia University Press. https://doi.org/10.1037/10584-006

Piek, J. P., Dawson, L., Smith, L. M., \& Gasson, N. (2008). The role of early fine and gross motor development on later motor and cognitive ability. Human Movement Science, 27(5), 668-681. https://doi.org/10.1016/j.humov.2007.11.002

Pittet, F., Coignard, M., Houdelier, C., Richard-Yris, M.-A., \& Lumineau, S. (2013). Effects of maternal experience on fearfulness and maternal behaviour in a precocial bird. Animal Behaviour, 85(4), 797-805.

Porterfield, J. H., Sindhurakar, A., Finley, J. M., \& Bradley, N. S. (2015). Drift during overground locomotion in newly hatched chicks varies with light exposure during embryogenesis. Developmental Psychobiology, 57(4), 459-469.

https://doi.org/10.1002/dev.21306

Pradhan, C., Wuehr, M., Akrami, F., Neuhaeusser, M., Huth, S., Brandt, T., ... Schniepp, R. (2015). Automated classification of neurological disorders of gait using spatiotemporal gait parameters. Journal of Electromyography and Kinesiology, 25(2), 413-422. https://doi.org/10.1016/J.JELEKIN.2015.01.004

Racine, H. L., Meadows, C. A., Ion, G., \& Serrat, M. A. (2018). Heat-induced limb length asymmetry has functional impact on weight bearing in mouse hindlimbs. Frontiers in Endocrinology, 9, 289. https://doi.org/10.3389/fendo.2018.00289

Redmer, D. A. A., Wallace, J. M. M., \& Reynolds, L. P. P. Effect of nutrient intake during pregnancy on fetal and placental growth and vascular development, 27 Domestic Animal Endocrinology § (2004). Elsevier.

Reed, W. L., \& Clark, M. E. (2011). Beyond maternal effects in birds: Responses of the embryo to the environment. In Integrative and Comparative Biology (Vol. 51, pp. 73-80). Narnia. https://doi.org/10.1093/icb/icr032

Represal, J., Van De Water, T. R., \& Bernd, P. (1991). Temporal pattern of nerve growth factor receptor expression in developing cochlear and vestibular ganglia in quail and mouse. Anatomy and Embryology, 184(5), 421-432. https://doi.org/10.1007/BF01236048

Reynolds, G. D., \& Lickliter, R. (2002). Effects of prenatal sensory stimulation on heart rate and behavioral measures of arousal in bobwhite quail embryos. Developmental Psychobiology, 41(2), 112-122. https://doi.org/10.1002/dev.10058 
Rhen, T., \& Lang, J. W. (2004). Phenotypic effect of incubation temperature in reptiles. In N. Valenzuela \& V. A. Lance (Eds.), Temperature Dependent Sex Determination in Vertebrates (pp. 90-98). Washington: Smithsonian Books. Retrieved from https://www.researchgate.net/publication/257429480

Robertson, I. S. (1961). The influence of turning on the hatchability of hens' eggs I. The effect of rate of turning on hatchability. The Journal of Agricultural Science, 57(1), 49-56. https://doi.org/10.1017/S0021859600050000

Robinson, J. S., Hartwich, K. ., Walker, S. ., Erwich, J. J., \& Owens, J. A. (1997). Early influences on embryonic and placental growth. Acta Paediatr Supplement, 423, $159-163$.

Robinson, S. (2005). Conjugate limb coordination after experience with an interlimb yoke: Evidence for motor learning in the rat fetus. Developmental Psychobiology, 47(4), 328-344. https://doi.org/10.1002/dev.20103

Rogers, L. J. (1990). Light input and the reversal of functional lateralization in the chicken brain. Behavioural Brain Research, 38(3), 211-221. https://doi.org/10.1016/0166-4328(90)90176-F

Rogers, L. J. (2014). Asymmetry of brain and behavior in animals: Its development, function, and human relevance. Genesis, 52(January), 555-571. https://doi.org/10.1002/dvg.22741

Rogers, L. J., Zucca, P., \& Vallortigara, G. (2004). Advantages of having a lateralized brain. Proceedings. Biological Sciences / The Royal Society, 271 Suppl, S420-S422. https://doi.org/10.1098/rsbl.2004.0200

Ronca, A. E., Fritzsch, B., Alberts, J. R., \& Bruce, L. L. (2000). Effects of microgravity on vestibular development and function in rats: Genetics and environment. Korean Journal of Biological Sciences, 4(3), 215-221. https://doi.org/10.1080/12265071.2000.9647547

Rosa, B. V, Firth, E. C., Blair, H. T., Vickers, M. H., H Morel, P. C., \& Pch, M. (2011). Voluntary exercise in pregnant rats positively influences fetal growth without initiating a maternal physiological stress response Downloaded from. Am J Physiol Regul Integr Comp Physiol, 300, 1134-1141. https://doi.org/10.1152/ajpregu.00683.2010.-The

Roy, S., Nag, T. C., Upadhyay, A. D., Mathur, R., \& Jain, S. (2014). Prenatal music stimulation facilitates the postnatal functional development of the auditory as well as visual system in chicks (Gallus domesticus). Journal of Biosciences, 39(1), 107117. https://doi.org/10.1007/s12038-013-9401-0

Rozenboim, I., Biran, I., Chaiseha, Y., Yahav, S., Rosenstrauch, A., Sklan, D., \& Halevy, 
O. (2004). The effect of a green and blue monochromatic light combination on broiler growth and development. Poultry Science, 83(5), 842-845.

https://doi.org/10.1093/ps/83.5.842

Rozenboim, I., Piestun, Y., Mobarkey, N., Barak, M., Hoyzman, A., \& Halevy, O. (2004). Monochromatic light stimuli during embryogenesis enhance embryo development and posthatch growth. Poultry Science, 83(8), 1413-1419. https://doi.org/10.1093/ps/83.8.1413

Ryu, Y. U., \& Bradley, N. S. (2009). Precocious locomotor behavior begins in the egg: Development of leg muscle patterns for stepping in the chick. PLOS ONE. https://doi.org/10.1371/journal.pone.0006111

Salvatore, D., Simonides, W. S., Dentice, M., Zavacki, A. M., \& Larsen, P. R. (2014). Thyroid hormones and skeletal muscle--new insights and potential implications. Nature Reviews. Endocrinology, 10(4), 206-214. https://doi.org/10.1038/nrendo.2013.238

Salvesen, K. Å., Hem, E., \& Sundgot-Borgen, J. (2012). Fetal wellbeing may be compromised during strenuous exercise among pregnant elite athletes. British Journal of Sports Medicine, 46(4), 279-283. https://doi.org/10.1136/bjsm.2010.080259

Sawacha, Z., Spolaor, F., Guarneri, G., Contessa, P., Carraro, E., Venturin, A., ... Cobelli, C. (2012). Abnormal muscle activation during gait in diabetes patients with and without neuropathy. Gait \& Posture, 35(1), 101-105. https://doi.org/10.1016/J.GAITPOST.2011.08.016

Schrijvers, D., Hulstijn, W., \& Sabbe, B. G. C. (2008). Psychomotor symptoms in depression: A diagnostic, pathophysiological and therapeutic tool. Journal of Affective Disorders, 109(1-2), 1-20. https://doi.org/10.1016/J.JAD.2007.10.019

Searls, D. T. (1964). The utilization of a known coefficient of variation in the estimation Procedure. Journal of the American Statistical Association, 59(308), 1225-1226. https://doi.org/10.1080/01621459.1964.10480765

Serrat, M. A. (2014). Environmental temperature impact on bone and cartilage growth. Comprehensive Physiology, 621-655. https://doi.org/10.1002/cphy.c130023

Sharp, A. A. (2015). Sensory regulation of spontaneous limb movements in the midstage embryonic chick. Developmental Psychobiology, 57(4), 385-396. https://doi.org/10.1002/dev.21292

Siegel, P. B., Isakson, S. T., Coleman, F. N., \& Huffman, B. J. (1969). Photoacceleration of development in chick embryos. Comparative Biochemistry and Physiology, 28(2), 753-758. https://doi.org/10.1016/0010-406X(69)92108-2 
Sindhurakar, A., \& Bradley, N. S. (2010). Kinematic analysis of overground locomotion in chicks incubated under different light conditions. Developmental Psychobiology, 52(8), 802-812. https://doi.org/10.1002/dev.20476

Sindhurakar, A., \& Bradley, N. S. (2012). Light accelerates morphogenesis and acquisition of interlimb stepping in chick embryos. PLoS ONE, 7(12), 1-14. https://doi.org/10.1371/journal.pone.0051348

Snijders, A. H., Takakusaki, K., Debu, B., Lozano, A. M., Krishna, V., Fasano, A., ... Hallett, M. (2016). Physiology of freezing of gait. Annals of Neurology, 80(5), 644659. https://doi.org/10.1002/ana.24778

Sommerville, J. A., Woodward, A. L., \& Needham, A. (2005). Action experience alters 3-month-old infants' perception of others' actions. Cognition, 96(1), B1-11. https://doi.org/10.1016/j.cognition.2004.07.004

Soska, K. C., Adolph, K. E., \& Johnson, S. P. (2010). Systems in development: motor skill acquisition facilitates three-dimensional object completion. Developmental Psychology, 46(1), 129-138. https://doi.org/10.1037/a0014618

Stockdale, F. E. (1997). Mechanisms of formation of muscle fiber types. Cell Structure and Function, 22, 37-43.

Stroobants, S., Gantois, I., Pooters, T., \& D'Hooge, R. (2013). Increased gait variability in mice with small cerebellar cortex lesions and normal rotarod performance.

Behavioural Brain Research, 241, 32-37.

https://doi.org/10.1016/J.BBR.2012.11.034

Suarez, M. E., Wilson, H. R., Mcpherson, B. N., Mather, F. B., \& Wilcox, C. J. (1996). Low temperature effects on embryonic development and hatch time. Poultry Science, 75(7), 924-932. https://doi.org/10.3382/ps.0750924

Szymanski, L. M., \& Satin, A. J. (2012). Strenuous exercise during pregnancy: is there a limit? American Journal of Obstetrics and Gynecology, 207(3), 179.e1-179.e6. https://doi.org/10.1016/j.ajog.2012.07.021

Tecott, L. H., \& Nestler, E. J. (2004). Neurobehavioral assessment in the information age. Nature Neuroscience, 7(5), 462-466. https://doi.org/10.1038/nn1225

Thompson, W., Russell, G., Baragwanath, G., Matthews, J., Vaidya, B., \& ThompsonCoon, J. (2018). Maternal thyroid hormone insufficiency during pregnancy and risk of neurodevelopmental disorders in offspring: A systematic review and metaanalysis. Clinical Endocrinology, 88(4), 575-584. https://doi.org/10.1111/cen.13550

Tilson, H. A. (1993). Neurobehavioral methods used in neurotoxicological research. Toxicology Letters, 68(1-2), 231-240. https://doi.org/10.1016/0378-4274(93)90134$\mathrm{J}$ 
Tona, K., Onagbesan, O., Bruggeman, V., Mertens, K., \& Decuypere, E. (2005). Effects of turning duration during incubation on embryo growth, utilization of albumen, and stress regulation. Poultry Science, 84(2), 315-320.

https://doi.org/10.1093/ps/84.2.315

Tona, K., Onagbesan, O., De Ketelaere, B., Decuypere, E., \& Bruggeman, V. (2003). Effects of turning duration during incubation on corticosterone and thyroid hormone levels, gas pressures in air cell, chick quality, and juvenile growth. Poultry Science, 82(12), 1974-1979. https://doi.org/10.1093/ps/82.12.1974

Tong, Q., McGonnell, I. M., Demmers, T. G. M., Roulston, N., Bergoug, H., Romanini, C. E., ... Exadaktylos, V. (2018). Effect of a photoperiodic green light programme during incubation on embryo development and hatch process. Animal, 12(4), 765773. https://doi.org/10.1017/S1751731117002117

Travers, B. G., Powell, P. S., Klinger, L. G., \& Klinger, M. R. (2013). Motor difficulties in autism spectrum disorder: Linking symptom severity and postural stability. Journal of Autism and Developmental Disorders, 43(7). https://doi.org/10.1007/s10803-012-1702-x

Tullett, S. G., \& Deeming, D. C. (1987). Failure to turn eggs during incubation: Effects on embryo weight, development of the chorioallantois and absorption of albumen. British Poultry Science, 28(2), 239-243. https://doi.org/10.1080/00071668708416958

Turkewitz, G., \& Kenny, P. A. (1982). Limitations on input as a basis for neural organization and perceptual development: A preliminary theoretical statement. Developmental Psychobiology, 15(4), 357-368. https://doi.org/10.1002/dev.420150408

Vallès, Y., \& Francino, M. P. (2018). Air Pollution, early life microbiome, and development. Current Environmental Health Reports, 5(4), 512-521. https://doi.org/10.1007/s40572-018-0215-y

Van Damme, R., Bauwens, D., Brana, F., \& Verheyen, R. F. (1992). Incubation temperature differentially affects hatching time, egg durvival, and hatchling performance in the lizard Podarcis Muralis. Herpetologica, 48(2), 220-228. Retrieved from https://www.jstor.org/stable/pdf/3892675.pdf

van der Pol, C. W., van Roovert-Reijrink, I. A. M., Maatjens, C. M., Gussekloo, S. W. S., Kranenbarg, S., Wijnen, J., ... van den Brand, H. (2019). Light-dark rhythms during incubation of broiler chicken embryos and their effects on embryonic and post hatch leg bone development. PLOS ONE, 14(1), e0210886. https://doi.org/10.1371/journal.pone.0210886

Wang, X., Jia, Q., Xiao, J., Jiao, H., \& Lin, H. (2015). Glucocorticoids retard skeletal 
muscle development and myoblast protein synthesis through a mechanistic target of rapamycin (mTOR)-signaling pathway in broilers ( Gallus gallus domesticus ).

Stress, 18(6), 686-698. https://doi.org/10.3109/10253890.2015.1083551

Wang, Z., Hallac, R. R., Conroy, K. C., White, S. P., Kane, A. A., Collinsworth, A. L., ... Mosconi, M. W. (2016). Postural orientation and equilibrium processes associated with increased postural sway in autism spectrum disorder (ASD). Journal of Neurodevelopmental Disorders, 8(1), 43. https://doi.org/10.1186/s11689-016-91781

Warne, R. T. (2014). A primer on multivariate analysis of variance (MANOVA) for behavioral scientists. Practical Assessment, Research and Evaluation, 19(17).

Webb, D. R. (1987). Thermal tolerance of avian embryos: A review. The Condor, 89(4), 874. https://doi.org/10.2307/1368537

While, G. M., Noble, D. W. A., Uller, T., Warner, D. A., Riley, J. L., Du, W.-G., \& Schwanz, L. E. (2018). Patterns of developmental plasticity in response to incubation temperature in reptiles. Journal of Experimental Zoology Part A: Ecological and Integrative Physiology, 329(4-5), 162-176. https://doi.org/10.1002/jez.2181

Willems, E, Decuypere, E., Buyse, J., \& Everaert, N. (2014). Importance of albumen during embryonic development in avian species, with emphasis on domestic chicken. Poultry Science Journal, 70. https://doi.org/10.1017/S0043933914000567

Willems, Els, Wang, Y., Koppenol, A., Lesuisse, J., Franssens, L., Decuypere, E., ... Buyse, J. (2015). Reduced protein availability by albumen removal during chicken embryogenesis decreases body weight and induces hormonal changes. Experimental Physiology, 100, 1298-1308. https://doi.org/10.1113/EP085313

Williams, J. L., Corbetta, D., \& Guan, Y. (2015). Learning to reach with "sticky" or "non-sticky" mittens: A tale of developmental trajectories. Infant Behavior and Development, 38, 82-96. https://doi.org/10.1016/j.infbeh.2015.01.001

Wilson, C. M., \& McNabb, F. M. (1997). Maternal thyroid hormones in Japanese quail eggs and their influence on embryonic development. General and Comparative Endocrinology, 107(2), 153-165. https://doi.org/10.1006/gcen.1997.6906

Wilson, H. R. (1991). Physiological requirements of the developing embryo: temperature and turning. In Avian incubation. Poultry Science Symposium 22 (pp. 145-156). Butterworth (Publishers) Ltd.

Wilson, H. R., Nesbeth, W. G., Miller, E. R., Ande, T. B., \& Ingram, D. R. (1979). Hatchability of bobwhite quail eggs incubated in various temperature combinations. Poultry Science, 58(5), 1351-1354. https://doi.org/10.3382/ps.0581351 
Woo, C. C., \& Leon, M. (2013). Environmental enrichment as an effective treatment for autism: A randomized controlled trial. Behavioral Neuroscience, 127(4), 487-497. https://doi.org/10.1037/a0033010

Wu, G., Bazer, F. W., Cudd, T. A., Meininger, C. J., \& Spencer, T. E. (2004). Maternal nutrition and fetal development. The Journal of Nutrition, 134(9), 2169-2172. https://doi.org/10.1093/jn/134.9.2169

Wu, K. C., Streicher, J., Lee, M. L., Hall, B. K., \& Müller, G. B. (2001). Role of motility in embryonic development I: Embryo movements and amnion contractions in the chick and the influence of illumination. Journal of Experimental Zoology, 291(2), 186-194. https://doi.org/10.1002/jez.1068

Yang, H., Xing, H., Wang, Z., Xia, J., Wan, Y., Hou, B., \& Zhang, J. (2015). Effects of intermittent lighting on Broiler growth performance, slaughter performance, serum biochemical parameters and tibia parameters. Italian Journal of Animal Science, 14(4), 4143. https://doi.org/10.4081/ijas.2015.4143

Yoshizaki, N., Ito, Y., Hori, H., Saito, H., \& Iwasawa, A. (2002). Absorption, transportation and digestion of egg white in quail embryos. Development, Growth and Differentiation, 44(1), 11-22. https://doi.org/10.1046/j.1440-169x.2002.00620.x

You, J.-Y., Chou, Y.-L., Lin, C.-J., \& Su, F.-C. (2001). Effect of slip on movement of body center of mass relative to base of support. Clinical Biomechanics, 16(2), 167173. https://doi.org/10.1016/S0268-0033(00)00076-0

Yu, Y., Li, Z., Zhong, Z., Jin, S., Pan, J., Rao, X., \& Yu, Y. (2018). Effect of Monochromatic Green LED Light Stimuli During Incubation on Embryo Growth, Hatching Performance, and Hormone Levels. Transactions of the ASABE, 61(2), 661-669. https://doi.org/10.13031/trans.12485

Zerbo, O., Iosif, A.-M., Walker, C., Ozonoff, S., Hansen, R. L., \& Hertz-Picciotto, I. (2013). Is maternal influenza or fever during pregnancy associated with autism or developmental delays? Results from the CHARGE (CHildhood Autism Risks from Genetics and Environment) Study. Journal of Autism and Developmental Disorders, 43(1), 25-33. https://doi.org/10.1007/s10803-012-1540-x

Zhang, H., Liu, S., Si, Y., Zhang, S., Tian, Y., Liu, Y., ... Zhu, Z. (2019). Natural sunlight plus vitamin D supplementation ameliorate delayed early motor development in newborn infants from maternal perinatal depression. Journal of Affective Disorders. https://doi.org/10.1016/j.jad.2019.07.010

Zhang, L., Zhang, H. J., Qiao, X., Yue, H. Y., Wu, S. G., Yao, J. H., \& Qi, G. H. (2012). Effect of monochromatic light stimuli during embryogenesis on muscular growth, chemical composition, and meat quality of breast muscle in male broilers. Poultry Science, 91(4), 1026-1031. https://doi.org/10.3382/ps.2011-01899 
Zhang, Lin, Wu, S., Wang, J., Qiao, X., Yue, H., Yao, J., ... Qi, G. (2014). Changes of plasma growth hormone, insulin-like growth factors-I, thyroid hormones, and testosterone concentrations in embryos and broiler chickens incubated under monochromatic green light. Italian Journal of Animal Science, 13(3), 3266. https://doi.org/10.4081/ijas.2014.3266

Zwicker, J. G., Missiuna, C., Harris, S. R., \& Boyd, L. A. (2012). Developmental coordination disorder: A review and update. European Journal of Paediatric Neurology : EJPN : Official Journal of the European Paediatric Neurology Society, 16(6), 573-581. https://doi.org/10.1016/j.ejpn.2012.05.005 
VITA

STARLIE C. BELNAP

ACEDEMIC PREPARATION AND PROFESSIONAL DEVELOPMENT

DEC 2019

CERTIFICATE IN ADVANCED QUANTIATIVE

ANALYSIS

Florida International University

Miami, FL

DEC 2019

DOCTOR OF PHILOSOPHY IN PSYCHOLOGY, specializing in Developmental Science

Florida International University

Miami, FL

MAR 2017

MASTERS IN PSYCHOLOGY, specializing in

Developmental Science

Florida International University

Miami, FL

DEC 2012

BACHELOR OF SCIENCE IN PSYCHOLOGY

Idaho State University

Pocatello, ID

$2019-2014$

GRADUATE RESEARCH LAB MANAGER

Developmental Psychobiology Lab

Florida International University

Miami, FL

2018-2019

LAB INSTRUCTOR

Research Methods II

Florida International University

Miami, FL

2014-2015

GRADUATE RESEARCH ASSISTANT

Hands Lab

Florida International University

Miami, FL

2013-2014

UNDERGRADUATE RESEARCH ASSISTANT

Developmental Behavioral Neuroscience Lab

Idaho State University

Pocatello, ID 


\section{PUBLICATIONS AND PRESENTATIONS}

Belnap, S.C., Vieira, E.R., Lickliter, R. (submitted, 2019) Effects of increased and decreased early incubation movement on neonatal precocial birds' locomotor behavior. Developmental Psychobiology

Belnap, S.C., Lickliter, R. (2019) Prenatal light exposure influences gait performance and body composition in bobwhite quail chicks. Physiology \& Behavior

Belnap, S.C., Lickliter, R. (2019) Incubation temperature affects neonatal precocial birds' coordinated motor behavior (Colinus virginianus). Physiology \& Behavior, 206(1), 51-58

Belnap, S.C., Lickliter, R. (2017) Coordinated movement is influenced by prenatal light experience in Bobwhite quail chicks (Colinus virginianus).

Behavioural Brain Research, 327, 103-111.

http://dx.doi.org/10.1016/j.bbr.2017.03.039

Boeving, E.R., Belnap, S.C., Nelson, E. (2017) Embraces are lateralized in spider monkeys (Ateles fusciceps rufiventris). American Journal of Primatology, 79(6), e22654.

Kauer S. D., Allmond J. T., Belnap S., \& Brumley, M. R. (2016). Maternal behavior influences development of a reflexive action pattern in the newborn rat. Developmental Psychobiology, 58(8), 1043-1054

Belnap, S. C., Allmond, J. T., Boomhower, S. R., Roberto, M. E., \& Brumley, M. R. (2014). Sensorimotor training during expression of the leg extension response (LER) in 1-day-old rats. Developmental Psychobiology, 56(7), 1553-1563.

Belnap, S.C., Lickliter, R. (2019) Incubation temperature influences fall frequency in Bobwhite quail neonates, The Society for Integrative and Comparative Biology, Tampa, FL

Belnap, S.C., Lickliter, R. (2018) Prenatal temperature influences the development of motor coordination in bobwhite quail hatchlings, Physical Therapy Brownbag, Florida International University, Miami, FL

Belnap, S.C., Lickliter, R. (2017) Prenatal light experience effects motor coordination in bobwhite quail, Graduate Student Appreciation Week, Miami, FL 